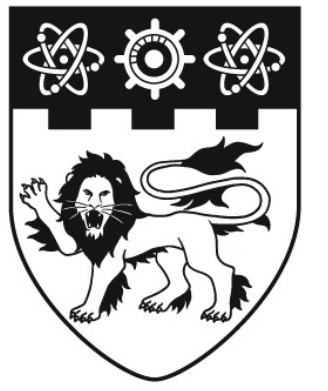

NANYANG UNIVERSITY

\title{
COMPLEX SYMMETRY \& WEIGHTED COMPOSITION OPERATORS ON FOCK SPACES
}

$<$
$\square$

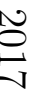

\section{PHAM VIET HAI}

School of Physical and Mathematical Sciences 



\section{COMPLEX SYMMETRY \& WEIGHTED COMPOSITION OPERATORS ON FOCK SPACES}

PHAM VIET HAI

School of Physical and Mathematical Sciences

A thesis submitted to the Nanyang Technological University in fulfillment of the requirement for the degree of Doctor of Philosophy 



\section{Acknowledgement}

I would like to express thanks to my supervisor Dr Le Hai Khoi for suggesting the topic, for the continuous support to my Ph.D. study and for his patience with immense knowledge. His guidance helped me in all the time of research and writing of this thesis.

I would like to thank Associate Professor Nicolas Privault in favor of my research. I would also like to thank all of the faculty and staff of the Division of Mathematical Sciences, School of Physical and Mathematical Sciences, Nanyang Technological University for providing me a good environment to study and research. A special thank to the NTU Research Scholarship and also the Merlion project 1.04.14 for the financial support.

Furthermore, I also use this opportunity to thank all members in the weekly Seminar Analysis of Dr Le Hai Khoi.

Last but not the least, I would like to thank my parents and my wife for having faith in me, and supporting spiritually me during the time of studying in Singapore as well as in my daily life. Clearly this thesis would not have been possible without their love and their unconditional support. 



\section{List of Works}

\section{Publications}

Below is the list of papers that have been published during my $\mathrm{PhD}$ studies.

[1] Pham Viet Hai, Le Hai Khoi, Complex symmetry of weighted composition operators on the Fock space, J. Math. Anal. Appl. 433 (2016), 1757-1771.

[2] Pham Viet Hai, Le Hai Khoi, Boundedness and compactness of weighted composition operators on Fock spaces $\mathcal{F}^{p}(\mathbb{C})$, Acta Math. Vietnam. 41 (2016), 531-537.

[3] Pham Viet Hai, Le Hai Khoi, Weighted composition operators that are complex symmetric on the Fock space $\mathcal{F}^{2}\left(\mathbb{C}^{n}\right)$, C. R. Math. Acad. Sci. Paris 354 (2016), 896900.

[4] Pham Viet Hai, Le Hai Khoi, Complex symmetric $C_{0}$-semigroups on the Fock space, J. Math. Anal. Appl. 445 (2017), 1367-1389.

[5] Pham Viet Hai, Le Hai Khoi, Complex symmetric weighted composition operators on the Fock space in several variables, to appear in Complex Var. Elliptic Equ. DOI: $10.1080 / 17476933.2017 .1315108$

\section{Conferences}

[6] Conference, Complex Symmetry in the Fock space, Lille 1 University - France, 5/2016 (Invited Speaker) 



\section{Abstract}

The main results of the thesis lie at the intersection of three areas: dynamical systems, complex symmetric operators, and weighted composition operators. We introduce two new concepts: weighted composition conjugations in operator theory, and complex symmetric $C_{0}$-semigroups $\left(C_{0}\right.$-groups $)$ in dynamical systems.

With the techniques of weighted composition operators, we solve completely the following problems on the Fock spaces $\mathcal{F}^{2}\left(\mathbb{C}^{n}\right)$ :

- the description of weighted composition operators which are conjugations;

- the criteria for bounded weighted composition operators to be complex symmetric.

For complex symmetric $C_{0}$-semigroups, we prove a new version of Stone's theorem:

- if each element of a $C_{0}$-semigroup is $\mathcal{C}$-symmetric with respect to a fixed conjugation $\mathcal{C}$, then the generator is $\mathcal{C}$-selfadjoint as an unbounded operator;

- and vice-versa, if the generator is $\mathcal{C}$-selfadjoint, then this $C_{0}$-semigroup is complex symmetric with respect to the conjugation $\mathcal{C}$.

More interestingly, we show that the class of complex symmetric $C_{0}$-groups contains unitary groups as a very particular case. Furthermore, we investigate this concept on the Fock space $\mathcal{F}^{2}(\mathbb{C})$ by making use of semigroups of weighted composition operators, and show that this a really generalization of unitary groups. 



\section{Notations}

$\mathbb{N}$

$\mathbb{R}_{+}$

$\mathbb{R}$

C

$\operatorname{Re}($.

$\operatorname{Im}($.

$\bar{z}$

$i$

$\mathrm{Cl}(M)$

$\mathbb{T}$

$\mathbb{D}$

$\mathbb{C}^{n}$

$I_{n}$

$\mathbb{B}_{n}$

$\operatorname{Hol}(G)$

$\operatorname{HSel}(G)$

const

0

1 set of non-negative integers

set of non-negative real numbers

set of real numbers

set of complex numbers

real part of a complex number

imaginary part of a complex number

conjugate number of a complex number $z$

complex unit $\left(i^{2}=-1\right)$

closure of a set $M$

unit circle

open unit disk

set of complex $n$-vectors

identity matrix with size $n \times n$

open unit ball of $\mathbb{C}^{n}$

set of all holomorphic functions on a domain $G \subseteq \mathbb{C}^{n}$

set of all holomorphic self-mappings of a domain $G \subseteq \mathbb{C}^{n}$

constant function

constant function with value 0

constant function with value 1 
- For integers $0 \leq k \leq m$, we write $m !=1.2 \cdots m$ (note that $0 !=1$ ), and

$$
\left(\begin{array}{c}
m \\
k
\end{array}\right)=\frac{m !}{k !(m-k) !}
$$

- Let $n \geq 1$ be a positive integer. For $z=\left(z_{1}, \ldots, z_{n}\right) \in \mathbb{C}^{n}$, we write $|z|=$ $\sqrt{\sum_{j=1}^{n}\left|z_{j}\right|^{2}}, \bar{z}=\left(\overline{z_{1}}, \ldots, \overline{z_{n}}\right)$, and $z^{\tau}=z_{1}^{\tau_{1}} \ldots z_{n}^{\tau_{n}}$, where $\tau=\left(\tau_{1}, \ldots, \tau_{n}\right)$ is a multi-index with $\tau_{j} \in \mathbb{N}, 1 \leq j \leq n$. The polynomial $P_{\tau}$ is defined by $P_{\tau}(z)=z^{\tau}$.

- For simplicity, inner products are always denoted by $\langle.,$.$\rangle , while norms are$ \|.\|. In addition, the symbol $\oplus$ stands for the orthogonal sum in Hilbert spaces.

- Given any Banach space $\mathbb{X}$ over field $\mathbb{C}$, the dual space $\mathbb{X}^{*}$ is defined as the set of all bounded linear functionals $f: \mathbb{X} \rightarrow \mathbb{C}$. A net $\left(T_{\alpha}\right)$ of bounded linear operators on $\mathbb{X}$ converges to $T$

i) in the strong operator topology if $\left\|T_{\alpha} x-T x\right\| \rightarrow 0$ for all $x \in \mathbb{X}$.

ii) in the weak operator topology if $\left|f\left(T_{\alpha} x\right)-f(T x)\right| \rightarrow 0$ for each $f \in \mathbb{X}^{*}$ and $x \in \mathbb{X}$.

For more details, please refer to the book [22, p. 183].

- Let $T$ be a linear operator from a linear subspace of a Banach space $\mathbb{X}$ over field $\mathbb{C}$, called the domain and denoted by $\operatorname{dom}(T)$, into $\mathbb{X}$. We use some standard notations concerning $T$ as follows.

$\begin{array}{ll}\operatorname{ker}(T) & \text { kernel of } T \\ \operatorname{Im}(T) & \text { image of } T \\ \sigma(T) & \text { spectrum of } T \\ \sigma_{p}(T) & \text { point spectrum of } T \\ T^{*} & \text { adjoint of } T\end{array}$

- We say that $T$ is bounded if $\operatorname{dom}(T)=\mathbb{X}$, and there exists a constant $M>0$ such that

$$
\|T x\| \leq M\|x\|, \quad \forall x \in \mathbb{X} .
$$


A bounded linear operator $T$ is compact on a Banach space $\mathbb{X}$ if for every bounded sequence $\left(x_{n}\right),\left(T x_{n}\right)$ has a convergent subsequence. In the case that $\mathbb{X}$ is reflexive, this definition is equivalent to the fact that the operator $T$ maps weakly convergent sequences into norm convergent sequences (see [22, p. 200]).

- For two unbounded linear operators $F: \operatorname{dom}(F) \subseteq \mathbb{X} \rightarrow \mathbb{X}$ and $G: \operatorname{dom}(G) \subseteq$ $\mathbb{X} \rightarrow \mathbb{X}$, the notation $F \preceq G$ means that $G$ is an extension of $F$ (see [24, p. 4]). If $F \preceq G$ and $G \preceq F$, then we write $F=G$. Furthermore, let $A, B$ be two bounded (linear or anti-linear) operators on $\mathbb{X}$, then we define the operator $A F B$ by

$$
\operatorname{dom}(A F B):=\{f \in \mathbb{X}: B f \in \operatorname{dom}(F)\}, \quad(A F B) f:=A(F(B f))
$$





\section{Contents}

1 Introduction 1

1.1 Complex symmetric operators .............. 1

1.2 Linear weighted composition operators . . . . . . . . . . 5

1.3 Semigroups of bounded linear operators . . . . . . . . . 6 6

1.4 Fock spaces of entire functions . . . . . . . . . . . . . . 9

1.4.1 One dimension . . . . . . . . . . . . . . . 10

1.4.2 Higher dimensions ................ 11

1.5 Structure of the thesis . . . . . . . . . . . . . 13

2 Complex symmetric bounded weighted composition operators on \begin{tabular}{ll}
$\mathcal{F}^{2}(\mathbb{C})$ & $\mathbf{1 5}$ \\
\hline
\end{tabular}

2.1 Anti-linear weighted composition operators . . . . . . . . . 16

2.1.1 Boundedness, isometry . . . . . . . . . . 19

2.1 .2 Involution . . . . . . . . . . . . . . . . . 28

2.1 .3 Conjugation . . . . . . . . . . . . . . 30

2.2 Linear weighted composition operators . . . . . . . . . . . 31

2.2.1 Some spectral properties . . . . . . . . . . . . 31

2.2.2 Complex symmetry with an arbitrary conjugation . . . . . 37

2.2.3 Complex symmetry with weighted composition conjugations 39

2.2.4 Normality . . . . . . . . . . . . . . . 45 
3 Complex symmetric $C_{0}$-semigroups 47

3.1 Motivation ................................. 48

3.2 Stone-type theorem . . . . . . . . . . . . . . . . . . 49

3.2.1 Solution to the first question . . . . . . . . . . . 49

3.2.2 Solution to the second question . . . . . . . . . 51

3.3 A new object: CSO $C_{0}$-semigroups ............. 53

3.4 Semigroup of weighted composition operators . . . . . . . . 57

$3.5 \mathcal{C}_{a, b, c^{-} \text {symmetric }} C_{0}$-semigroups on $\mathcal{F}^{2}(\mathbb{C}) \ldots \ldots \ldots$

3.5.1 A strong continuity of semigroups of weighted composition operators ...................... 62

3.5.2 A characterization of CSO $C_{0}$-semigroups . . . . . . . . 70

3.5.3 Generators of CSO $C_{0}$-semigroups . . . . . . . . . . . 70

3.6 Unitary $C_{0}$-semigroups on $\mathcal{F}^{2}(\mathbb{C}) \ldots \ldots \ldots$

3.6.1 A characterization of unitary $C_{0}$-semigroups $\ldots \ldots$

3.6.2 Generators of unitary $C_{0}$-semigroups . . . . . . . . 81

4 Complex symmetric bounded weighted composition operators on $\mathcal{F}^{2}\left(\mathbb{C}^{n}\right)$

4.1 Anti-linear weighted composition operators . . . . . . . . 86

4.1.1 Bounded, invertible, and unitary properties . . . . . . 89

4.1.2 Selfadjoint property . . . . . . . . . . . . 93

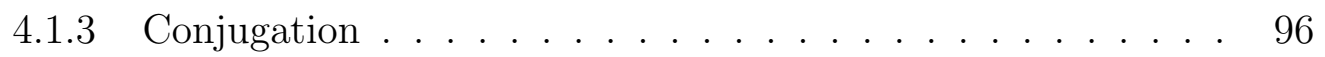

4.1 .4 Involutive property . . . . . . . . . . . . 97

4.2 Linear weighted composition operators . . . . . . . . . . . . 100

4.2.1 Complex symmetry with weighted composition conjugations 100

4.2 .2 Normality . . . . . . . . . . . . . . . 103 
5 Conclusion \& Future Works 107

5.1 Complex symmetry in other function spaces . . . . . . . . . . . . 107

5.2 Complex symmetry of unbounded operators . . . . . . . . . . 108

Bibliography

109

Appendices

Appendix

A A study on the Fock spaces $\mathcal{F}^{p}(\mathbb{C})$

A.1 Boundedness on $\mathcal{F}^{p}(\mathbb{C}) \ldots \ldots \ldots$. . . . . . . . . . . . . . . . .

A.2 Compactness on $\mathcal{F}^{p}(\mathbb{C}) \ldots \ldots \ldots \ldots$ 



\section{Chapter 1}

\section{Introduction}

\subsection{Complex symmetric operators}

The theory of complex symmetric operators has been investigated, linking to many areas of complex analysis, operators theory, and specially to mathematical physics. Its general study was initiated by Garcia and Putinar in [9, 10] with the motivation arising in matrix analysis and function theory. Complex symmetric operators are becoming an important class in operator theory, which is large enough to cover many well-known operators occurring in applications, for instance: selfadjoint operators, unitary operators, normal operators, etc. The survey [8] is a good source providing progresses on the theory as well as applications in quantum physics.

In the beginning, we recall basic terminologies used in the thesis. Let $\mathcal{H}$ be a complex separable Hilbert space endowed with inner product $\langle.,$.$\rangle .$

Definition 1.1.1. A mapping $A: \mathcal{H} \rightarrow \mathcal{H}$ is called anti-linear if

$$
A(a x+b y)=\bar{a} A(x)+\bar{b} A(y), \forall x, y \in \mathcal{H}, \forall a, b \in \mathbb{C} .
$$

The boundedness of anti-linear operators is defined similarly as for linear operators, while for the adjoint operation there is a slight difference. 
Definition 1.1.2. Consider a bounded anti-linear operator $A: \mathcal{H} \rightarrow \mathcal{H}$. Then the adjoint of $A$ is the bounded anti-linear $A^{*}: \mathcal{H} \rightarrow \mathcal{H}$ satisfying

$$
\langle A x, y\rangle=\left\langle A^{*} y, x\right\rangle, \quad \forall x, y \in \mathcal{H}
$$

As for linear operators, the adjoint of a bounded anti-linear operator always exists uniquely by the Riesz representation lemma. Although linear and antilinear operators are distinguished, both they have a common definition in several situations.

Definition 1.1.3. We say that a bounded (linear or anti-linear) operator $A: \mathcal{H} \rightarrow$ $\mathcal{H}$ is

1. selfadjoint if $A=A^{*}$;

2. invertible if there exists a bounded (linear or anti-linear respectively) $B$ such that $A B=B A=I$, the identity operator on $\mathcal{H}$. In this case, we write $A^{-1}=B$

3. unitary if $A^{-1}=A^{*}$;

4. normal if it commutes with its adjoint, that is $A A^{*}=A^{*} A$.

For unbounded linear operators, selfadjointness is defined as follows.

Definition 1.1.4. Let $A: \operatorname{dom}(A) \subseteq \mathcal{H} \rightarrow \mathcal{H}$ be a closed, densely defined, linear operator. We say that the operator $A$ is

1. symmetric if $A \preceq A^{*}$;

2. selfadjoint if $A=A^{*}$.

Both cases are called jointly as Hermitian operators. 
Clearly, selfadjoint operators always are symmetric, but the reverse statement fails to hold. Namely, there always exists a symmetric operator, which is not selfadjoint. Interestingly, the Von Neumann theory of selfadjoint extensions allows us to construct selfadjoint operators from a symmetric operator, (see [24, Chapter $13])$.

We now pass to conjugations, which are among the indispensable features in defining complex symmetric operators.

Definition 1.1.5. An anti-linear mapping $\mathcal{C}: \mathcal{H} \rightarrow \mathcal{H}$ is called a conjugation, if it is

1. involutive: $\mathcal{C}^{2}=I$;

2. isometric: $\|\mathcal{C} u\|=\|u\|, \forall u \in \mathcal{H}$.

It turns out that by the polarization identity

$$
4\langle u, v\rangle=\|u+v\|^{2}+i\|u+i v\|^{2}-\|u-v\|^{2}-i\|u-i v\|^{2},
$$

axiom (2) is equivalent to the fact that

$$
\langle\mathcal{C} u, \mathcal{C} v\rangle=\langle v, u\rangle, \quad \forall u, v \in \mathcal{H}
$$

Just as Hermitian operators, the complex symmetry also is classified into two definitions: one for bounded operators and another one for unbounded operators.

- In the boundedness case, we have:

Definition 1.1.6. Let $T: \mathcal{H} \rightarrow \mathcal{H}$ be a bounded linear operator and $\mathcal{C}: \mathcal{H} \rightarrow \mathcal{H}$ a conjugation. We say that $T$ is $\mathcal{C}$-symmetric if $T=\mathcal{C} T^{*} \mathcal{C}$. This means that

$$
T f=\mathcal{C} T^{*} \mathcal{C} f, \forall f \in \mathcal{H} .
$$

Particularly this case, we often use the following note in practice: 
Lemma 1.1.7. If a bounded linear operator is complex symmetric on a dense subset, then it is complex symmetric on the whole Hilbert space $\mathcal{H}$.

When $\mathcal{H}=\mathbb{C}^{n}(n \geq 1)$, it is proved that a square matrix is symmetric if and only if it is complex symmetric with the standard conjugation defined by

$$
\kappa\left(z_{1}, z_{2}, \ldots, z_{n}\right)=\left(\overline{z_{1}}, \overline{z_{2}}, \ldots, \overline{z_{n}}\right)
$$

This shows that complex matrices are a particular case of complex symmetric operators. Furthermore, every finite square matrix is similar to a complex symmetric matrix.

- For unbounded operators, we have:

Definition 1.1.8. Let $T: \operatorname{dom}(T) \subseteq \mathcal{H} \rightarrow \mathcal{H}$ be a closed, densely defined, linear operator and $\mathcal{C}: \mathcal{H} \rightarrow \mathcal{H}$ a conjugation. We say that $T$ is

1. $\mathcal{C}$-symmetric if $T \preceq \mathcal{C} T^{*} \mathcal{C}$. This means that

$$
\mathcal{C} \operatorname{dom}(T) \subseteq \operatorname{dom}\left(T^{*}\right), \quad T f=\mathcal{C} T^{*} \mathcal{C} f, \forall f \in \operatorname{dom}(T)
$$

2. $\mathcal{C}$-selfadjoint if $T=\mathcal{C} T^{*} \mathcal{C}$. This means that

$$
\mathcal{C} \operatorname{dom}(T)=\operatorname{dom}\left(T^{*}\right), \quad T f=\mathcal{C} T^{*} \mathcal{C} f, \forall f \in \operatorname{dom}(T) .
$$

Both cases are called jointly as complex symmetric operators.

In comparison with bounded ones, complex symmetric unbounded operators have received a little attention. To the best of our knowledge, there seem to be very few articles studying these operators. The majority of articles, so far as we know, are devoted to differential operators. The pioneering works of Glazman [11, 12] with its complement [16, 21] give an analogue of the Von Neumann theory. 


\subsection{Linear weighted composition operators}

In this section, $\mathbb{X}$ is a Banach space (or Hilbert space when discussing the complex symmetry) of holomorphic functions on a domain $G \subseteq \mathbb{C}^{n}$ (see [3, Definition 1.1]). Let $W_{\psi, \varphi}$ be a weighted composition operator on $\mathbb{X}$, induced by $\psi \in \operatorname{Hol}(G)$ and $\varphi \in \operatorname{HSel}(G)$, with the domain

$$
\operatorname{dom}\left(W_{\psi, \varphi}\right)=\{f \in \mathbb{X}: \psi \cdot f \circ \varphi \in \mathbb{X}\}
$$

The action of $W_{\psi, \varphi}$ on each element $f \in \operatorname{dom}\left(W_{\psi, \varphi}\right)$ is

$$
\left(W_{\psi, \varphi} f\right)(z)=\psi(z) f(\varphi(z)), \quad \forall z \in G
$$

The functions $\psi$ and $\varphi$ are called the symbols of $W_{\psi, \varphi}$. When $\psi \equiv \mathbf{1}$, we call simply as the composition operator and denote by $C_{\varphi}$. Throughout the paper, we always assume that $\psi \not \equiv \mathbf{0}$. It is clear that the operator $W_{\psi, \varphi}$ is linear, and $\operatorname{dom}\left(W_{\psi, \varphi}\right)$ is a non-empty subspace of $\mathbb{X}\left(\right.$ since $\left.\mathbf{0} \in \operatorname{dom}\left(W_{\psi, \varphi}\right)\right)$. In general, $\operatorname{dom}\left(W_{\psi, \varphi}\right)$ is a proper subspace of $\mathbb{X}$.

Note that the operator $W_{\psi, \varphi}$ is bounded on $\mathbb{X}$ if $\operatorname{dom}\left(W_{\psi, \varphi}\right)=\mathbb{X}$, and there exists a constant $M$ such that

$$
\left\|W_{\psi, \varphi} f\right\| \leq M\|f\|, \quad \forall f \in \mathbb{X} .
$$

During the past several decades, the interaction between the operator theoretic properties of $W_{\psi, \varphi}$ and the function theoretic properties of the symbols $\psi$ and $\varphi$ (such as boundedness, compactness, compact difference, essential norm, etc.) always receives a special interest by many researchers. The monograph [3] is a good source to learn more recent results. Meanwhile, a study of the complex symmetry for weighted composition operators has only recently started.

In order to call investigations, Garcia and Hammond published the paper [7] with the title being a question for the operator theory community: 
A. Which weighted composition operators are complex symmetric?

In general, this is undoubtedly a difficult question. In the initial stage, researchers studied this question for Hardy spaces with either special conjugations or special symbols. Garcia and Hammond explicitly characterized in [7] all weighted composition operators on weighted Hardy spaces, which are complex symmetric with respect to the standard conjugation

$$
\mathcal{J} f(z)=\overline{f(\bar{z})}, \quad z \in \mathbb{D} .
$$

With the same conjugation, Jung and colleagues investigated independently in [15] for the classical Hardy space. In [27], Wang and Yao extended some results to Hardy spaces of holomorphic functions on the unit ball $\mathbb{B}_{n}$ with the same kind conjugation which is defined in the multi-dimensional case

$$
\mathcal{K} g(w)=\overline{g(\bar{w})}, \quad w \in \mathbb{B}_{n}(n \geq 1) .
$$

Aside from these investigations, several authors [1, 5, 6, 19] took considerable effort to settle the automorphic and linear fractional symbols $\varphi$. The general problem is still challenging to the operator theory community, even in the case of composition operators $C_{\varphi}$.

\subsection{Semigroups of bounded linear operators}

The theory of semigroups of bounded linear operators on Banach spaces was established by the early 1950s with the motivation coming from many areas of mathematics, for instance: functional equations, differential equations, etc. With the contribution of many authors in the 1970s and 80s, the theory achieved a certain perfection. 
Definition 1.3.1. A family $(T(s))_{s \geq 0}$ of bounded linear operators on a Banach space $\mathbb{X}$, is called a semigroup (or linear dynamical system) on $\mathbb{X}$, if it is a representation of a semigroup $\left(\mathbb{R}_{+},+\right)$. Namely,

1. $T(0)=I$, the identity operator on $\mathbb{X}$;

2. $T(t+s)=T(t) T(s)$, for every $t, s \geq 0$.

Axiom (2) means that $T(t+s)$ is a composite operator of $T(t)$ and $T(s)$, that is

$$
T(t+s) x=T(t)(T(s) x), \quad \forall x \in \mathbb{X}
$$

Consequently, two arbitrary elements of a semigroup are always commutative. Equipping the continuity in the strong operator topology for semigroups gives the following definition.

Definition 1.3.2. A semigroup $(T(s))_{s \geq 0}$ of bounded linear operators on $\mathbb{X}$ is called strongly continuous if it is continuous in the strong operator topology. Namely,

3. $\lim _{s \rightarrow 0^{+}} T(s) x=x$, for every $x \in \mathbb{X}$.

In this case, we call $(T(s))_{s \geq 0}$ strongly continuous semigroup, or $C_{0}$-semigroup.

Interestingly, a semigroup is strongly continuous if and only if it is weakly continuous. This means that the axiom above can be replaced by

$$
\lim _{s \rightarrow 0^{+}} f(T(s) x)=f(x), \quad \forall f \in \mathbb{X}^{*}, \forall x \in \mathbb{X} .
$$

Note that using the continuity at every point in $\mathbb{R}_{+}$instead of the continuity at 0 does not change the class of semigroups. That is axiom in Definition 1.3 .2 also can be restated as

$$
\lim _{t \rightarrow t_{0}} T(t) x=T\left(t_{0}\right) x, \quad \forall x \in \mathbb{X}, \forall t_{0} \geq 0 .
$$

The following definition plays an important part in linking to evolution equations. 
Definition 1.3.3. The generator $A: \operatorname{dom}(A) \subseteq \mathbb{X} \rightarrow \mathbb{X}$ of a $C_{0}$-semigroup $(T(s))_{s \geq 0}$ is an operator $A$ defined by

$$
A x=\lim _{s \rightarrow 0^{+}} \frac{T(s) x-x}{s},
$$

with $\operatorname{dom}(A)$ consisting of those $x$ for which the limit exists.

We note that a generator of a $C_{0}$-semigroup is always a closed and densely defined linear operator that determines the semigroup uniquely. This means that for each $x \in \operatorname{dom}(A)$ the function $u: t \mapsto u(t):=T(t) x$ is the unique classical solution of the abstract Cauchy problem

$$
w^{\prime}(s)=A w(s), s \geq 0, \quad w(0)=x .
$$

Passing to the adjoint operation, axioms (1)-(2) in Definition 1.3.1 show that the family $\left(T(s)^{*}\right)_{s \geq 0}$ of adjoint operators $T(s)^{*}$ is a semigroup on the dual space $\mathbb{X}^{*}$. In general, it is not necessarily strongly continuous, but on a reflexive Banach space $\mathbb{X},\left(T(s)^{*}\right)_{s \geq 0}$ is again a $C_{0^{-}}$-semigroup. In particular, on Hilbert spaces, $\left(T(s)^{*}\right)_{s \geq 0}$ is always strongly continuous, and furthermore, its generator is $A^{*}$. The reader can find the detailed proof in [20, Theorem 2.2.8].

If the axioms in Definitions 1.3.1 1.3.2 hold for $\mathbb{R}$ instead of $\mathbb{R}_{+}$, we call $(T(s))_{s \in \mathbb{R}}$ a group, $C_{0}$-group, respectively. The generator of a $C_{0}$-group $(T(s))_{s \in \mathbb{R}}$ is defined slightly differently as follows

$$
A x=\lim _{s \rightarrow 0} \frac{T(s) x-x}{s},
$$

with $\operatorname{dom}(A)$ including elements for which the limit exists. Given a $C_{0}$-group $(V(s))_{s \in \mathbb{R}}$ with the generator $(A, \operatorname{dom}(A))$, we can define $V_{+}(s):=V(s)$ and $V_{-}(s):=V(-s)$ for $s \geq 0$. As is well known, $\left(V_{+}(s)\right)_{s \geq 0}$ and $\left(V_{-}(s)\right)_{s \geq 0}$ are $C_{0}$-semigroups with generators $A$ and $-A$, respectively, see [4, p. 79].

We refer the reader to the monograph [4] for more information on semigroups as well as groups. 


\subsection{Fock spaces of entire functions}

In this section, we recall some basic facts about the Fock spaces, and recent results studying the operator theoretic properties of weighted composition operators on these spaces.

Let $n \geq 1$ be a natural number. The Fock space $\mathcal{F}^{2}\left(\mathbb{C}^{n}\right)$ consists of all $f \in$ $\operatorname{Hol}\left(\mathbb{C}^{n}\right)$ for which

$$
\|f\|=\left(\frac{1}{\pi^{n}} \int_{\mathbb{C}^{n}}|f(z)|^{2} e^{-|z|^{2}} d V(z)\right)^{1 / 2}<\infty,
$$

where $d V$ is the Lebesgue measure on $\mathbb{C}^{n}$. It is a Hilbert space with the following inner product

$$
\langle f, g\rangle=\frac{1}{\pi^{n}} \int_{\mathbb{C}^{n}} f(z) \overline{g(z)} e^{-|z|^{2}} d V(z) .
$$

Moreover, it is a reproducing kernel Hilbert space with the kernel function $K_{z}(u)=$ $e^{\langle u, z\rangle}$. Namely,

$$
f(z)=\left\langle f, K_{z}\right\rangle, \quad \forall z \in \mathbb{C}^{n}, \forall f \in \mathcal{F}^{2}(\mathbb{C}) .
$$

As is well known, the set of all $z^{\tau}$ (here $\tau=\left(\tau_{1}, \cdots, \tau_{n}\right)$ is a multi-index and $z=$ $\left.\left(z_{1}, \cdots, z_{n}\right)\right)$ is an orthogonal basis of $\mathcal{F}^{2}\left(\mathbb{C}^{n}\right)$, while the span of kernel functions is dense. A direct computation gives

$$
\left\|K_{z}\right\|=e^{|z|^{2} / 2}, \quad\left\|z^{\tau}\right\|=\sqrt{\tau_{1} ! \ldots \tau_{n} !}
$$

Since $h(z)=\left\langle h, K_{z}\right\rangle$, we always have

$$
|h(z)| \leq\|h\| e^{|z|^{2} / 2}, \quad \forall z \in \mathbb{C}^{n}, \forall h \in \mathcal{F}^{2}\left(\mathbb{C}^{n}\right)
$$

For each $z \in \mathbb{C}^{n}$, we put

$$
k_{z}=\frac{K_{z}}{\left\|K_{z}\right\|},
$$

which is a unit vector in $\mathcal{F}^{2}\left(\mathbb{C}^{n}\right)$. 
Let $W_{\psi, \varphi}$ be a linear weighted composition operator, induced by $\psi \in \operatorname{Hol}\left(\mathbb{C}^{n}\right) \backslash$ $\{\mathbf{0}\}$ and $\varphi \in \operatorname{HSel}\left(\mathbb{C}^{n}\right)$, that is $W_{\psi, \varphi} f=\psi \cdot f \circ \varphi$. The last decade witnessed many important contributions to studying the operator theoretic properties of $W_{\psi, \varphi}$ on Fock spaces. In this section, we summarize several results needed in the sequel. In order for the reader to see the difficulty between one dimension and multidimensions, we divide into two subsections corresponding to these two cases.

\subsubsection{One dimension}

The most simple case, but not less important, is the one dimension. In this case, the symbol $\psi \in \operatorname{Hol}(\mathbb{C}) \backslash\{\mathbf{0}\}$, while $\varphi \in \operatorname{HSel}(\mathbb{C}) \equiv \operatorname{Hol}(\mathbb{C})$.

Recently, Le [18] characterized boundedness as well as compactness of $W_{\psi, \varphi}$ on the Fock space $\mathcal{F}^{2}(\mathbb{C})$ in terms of the behavior properties (such as boundedness, convergence to 0) of the expression

$$
|\psi(z)| \cdot \frac{\left\|K_{\varphi(z)}\right\|}{\left\|K_{z}\right\|}, \quad z \in \mathbb{C}
$$

For convenience, we put

$$
M_{z}(\psi, \varphi):=\left(|\psi(z)| \cdot \frac{\left\|K_{\varphi(z)}\right\|}{\left\|K_{z}\right\|}\right)^{2}=|\psi(z)|^{2} e^{|\varphi(z)|^{2}-|z|^{2}}, \quad z \in \mathbb{C},
$$

and $M(\psi, \varphi):=\sup _{z \in \mathbb{C}} M_{z}(\psi, \varphi)$.

Proposition 1.4.1 ([18]). The operator $W_{\psi, \varphi}: \mathcal{F}^{2}(\mathbb{C}) \rightarrow \mathcal{F}^{2}(\mathbb{C})$ is

1. bounded if and only if $\psi \in \mathcal{F}^{2}(\mathbb{C})$ and $M(\psi, \varphi)<\infty$. In this case, $\varphi(z)=$ $a z+b$ with $|a| \leq 1$.

2. compact if and only if $\psi \in \mathcal{F}^{2}(\mathbb{C})$ and $\lim _{|z| \rightarrow \infty} M_{z}(\psi, \varphi)=0$. In this case, $\varphi(z)=a z+b$ with $|a|<1$.

Here it should be noted that the key technique to show that $\varphi$ is affine is the following lemma. 
Lemma 1.4.2 ([18]). Let $\psi$ and $\varphi$ be two entire functions with $\psi \not \equiv \mathbf{0}$. Suppose that $M(\psi, \varphi)$ is finite. Then $\varphi(z)=a z+b$ with $|a| \leq 1$. In addition, if $|a|=1$, then $\psi(z)=\psi(0) e^{-a \bar{b} z}$.

In addition, he also investigated the normality of bounded weighted composition operators on $\mathcal{F}^{2}(\mathbb{C})$.

Proposition 1.4.3 ([18]). The operator $W_{\psi, \varphi}: \mathcal{F}^{2}(\mathbb{C}) \rightarrow \mathcal{F}^{2}(\mathbb{C})$ is a normal bounded operator if and only if

1. $\varphi(z)=a z+b$ with $|a|<1$ and $\psi(z)=\psi(0) e^{d z}$, where $d=\bar{b}(1-a)(1-\bar{a})^{-1}$.

2. $\varphi(z)=a z+b$ with $|a|=1$ and $\psi(z)=\psi(0) e^{d z}$, where $d=-a \bar{b}$.

Note that in [18] a description of all isometric weighted composition operators on $\mathcal{F}^{2}(\mathbb{C})$ is also given. Recently, some of these results have been generalized to the case of higher dimensions in [30].

\subsubsection{Higher dimensions}

We note that in the case of one dimension, boundedness was characterized completely, but for the multi-dimensional case $\mathcal{F}^{2}\left(\mathbb{C}^{n}\right)$, this topic still calls for investigation. We collect here the answers in some particular cases.

For $\psi \equiv \mathbf{1}$, Carswell and colleagues succeeded to identify all bounded composition operators.

Proposition 1.4.4 ([2]). The operator $C_{\varphi}: \mathcal{F}^{2}\left(\mathbb{C}^{n}\right) \rightarrow \mathcal{F}^{2}\left(\mathbb{C}^{n}\right)$ is

1. bounded if and only if $\varphi(z)=Q z+p$, where $Q$ is a linear operator on $\mathbb{C}^{n}$ with $\|Q\| \leq 1, p \in \mathbb{C}^{n}$ and

$$
\langle Q x, p\rangle=0 \quad \text { whenever } x \in \mathbb{C}^{n} \text { with }|Q x|=|x| .
$$


2. compact if and only if $\varphi(z)=Q z+p$, where $Q$ is a linear operator on $\mathbb{C}^{n}$ with $\|Q\|<1$, and $p \in \mathbb{C}^{n}$.

Following Le's ideas, Zhao [29] extended some of those results to $\mathcal{F}^{2}\left(\mathbb{C}^{n}\right)$, under the additional assumption that the symbol function $\varphi(z)=Q z+p$ is injective. This assumption is equivalent to the invertibility of $Q$.

To state these results, which are summarized below, we also use the notations $M(\psi, \varphi)$ and $M_{z}(\psi, \varphi)$ as for the case of one dimension, and moreover we put

$$
m(\psi, \varphi):=\inf _{z \in \mathbb{C}^{n}} M_{z}(\psi, \varphi)
$$

Proposition 1.4.5 ([29]). 1. If the operator $W_{\psi, \varphi}$ is bounded on $\mathcal{F}^{2}\left(\mathbb{C}^{n}\right)$, then there exists a linear operator $Q$ on $\mathbb{C}^{n}$ with $\|Q\| \leq 1$ and $p \in \mathbb{C}^{n}$, such that $\varphi(z)=Q z+p$ and $M(\psi, \varphi)<\infty$. In particular, when $Q$ is invertible, these two conditions together are sufficient also.

2. The operator $W_{\psi, \varphi}$ is bounded invertible on $\mathcal{F}^{2}\left(\mathbb{C}^{n}\right)$, if and only if there exists an invertible linear operator $Q$ on $\mathbb{C}^{n}$ with $\|Q\|=1, p \in \mathbb{C}^{n}$, such that $\varphi(z)=Q z+p$ and $0<m(\psi, \varphi) \leq M(\psi, \varphi)<\infty$.

Just as for the case of one variable, the following lemma, which is a generalization of Lemma 1.4 .2 is useful in showing that the symbol $\varphi$ is affine.

Lemma 1.4.6 $([29])$. Let $\psi \in \operatorname{Hol}\left(\mathbb{C}^{n}\right)$ and $\varphi \in \operatorname{HSel}\left(\mathbb{C}^{n}\right)$. If $M(\psi, \varphi)<\infty$, then $\varphi(z)=Q z+p$, where $Q$ is a linear operator on $\mathbb{C}^{n}$ with $\|Q\| \leq 1$ and $p \in \mathbb{C}^{n}$. When $Q$ is unitary, there exists a constant $c \in \mathbb{C} \backslash\{0\}$ such that $\psi(z)=c e^{-\langle Q z, p\rangle}$.

Zhao also characterized in his framework isometric as well as unitary weighted composition operators. It turns out that these operators have a common structure as follows.

Proposition 1.4.7 ([28, 30]). The following statements are equivalent. 
1. The operator $W_{\psi, \varphi}$ is isometric on $\mathcal{F}^{2}\left(\mathbb{C}^{n}\right)$.

2. The operator $W_{\psi, \varphi}$ is unitary on $\mathcal{F}^{2}\left(\mathbb{C}^{n}\right)$.

3. The functions $\psi, \varphi$ are of forms

$$
\varphi(z)=Q z+p, \psi(z)=c e^{-\langle Q z, p\rangle-|p|^{2} / 2}, \quad z \in \mathbb{C}^{n}
$$

where $Q$ is an unitary linear operator on $\mathbb{C}^{n}, p \in \mathbb{C}^{n}$, and $c \in \mathbb{C}$ with $|c|=1$.

We note that the following identity plays an important role in proving the necessities of Propositions 1.4.5 1.4.7 if the operator $W_{\psi, \varphi}$ is bounded on $\mathcal{F}^{2}\left(\mathbb{C}^{n}\right)$, then

$$
W_{\psi, \varphi}^{*} K_{z}=\overline{\psi(z)} K_{\varphi(z)}, \quad \forall z \in \mathbb{C}^{n}
$$

\subsection{Structure of the thesis}

The thesis is organized as follows.

Chapter 2 is devoted to studying question A. First, we give a method to construct new conjugations. This method is motivated, on one hand, by the fact that the operator $\mathcal{J}$ defined by $(1.4)$ is also a conjugation on $\mathcal{F}^{2}(\mathbb{C})$, and on the other hand, by noticing that $\mathcal{J}$ is just a very particular case of the so-called anti-linear weighted composition operators.

Since an anti-linear operator is a conjugation if and only if it is both isometric and involutive, we characterize all isometric as well as all involutive anti-linear weighted composition operators on $\mathcal{F}^{2}(\mathbb{C})$, and then combine them to obtain the so-called weighted composition conjugations. Having constructed conjugations, we characterize bounded weighted composition operators, which are complex symmetric. 
In Chapter 3, we introduce a new concept in dynamical systems: complex symmetric $C_{0}$-semigroups $\left(C_{0}\right.$-groups), which contains unitary groups as a very particular case. We show that if a $C_{0}$-semigroup is also $\mathcal{C}$-symmetric with respect to a fixed conjugation $\mathcal{C}$, then the generator is $\mathcal{C}$-selfadjoint as an unbounded operator, and vice-versa, if the generator is $\mathcal{C}$-selfadjoint, then this $C_{0}$-semigroup is complex symmetric with respect to the conjugation $\mathcal{C}$. The results obtained in Chapter 2 allow us to study complex symmetric $C_{0}$-semigroups on the Fock space $\mathcal{F}^{2}(\mathbb{C})$. In particular, we obtain another class of complex symmetric operators on $\mathcal{F}^{2}(\mathbb{C})$, which are generators of complex symmetric $C_{0}$-semigroups. Note that such operators are unbounded and do not coincide with weighted composition operators.

Chapter 4 studies weighted composition conjugations in the case of higher dimensions, with a different approach. It is inspired by the following note: an antilinear mapping is a conjugation if and only if it is both unitary and selfadjoint. Instead of characterizing isometric and involutive anti-linear weighted composition operators, we characterize all selfadjoint as well as all unitary ones to get weighted composition conjugations on $\mathcal{F}^{2}\left(\mathbb{C}^{n}\right)$. With these conjugations, we give the description of bounded weighted composition operators which are complex symmetric on $\mathcal{F}^{2}\left(\mathbb{C}^{n}\right)$.

Finally, in Appendix, we give characterizations for boundedness and compactness of weighted composition operators on the more general Fock spaces $\mathcal{F}^{p}(\mathbb{C})$ with $p>0$. Our results contains the corresponding results for $\mathcal{F}^{2}(\mathbb{C})$ as a particular case. 


\section{Chapter 2}

\section{Complex symmetric bounded}

\section{weighted composition operators}

on $\mathcal{F}^{2}(\mathbb{C})$

In this chapter, we investigate a complex symmetry of weighted composition operators on the Fock space $\mathcal{F}^{2}(\mathbb{C})$.

In the first section, we construct new conjugations by making use of anti-linear weighted composition operators. We note that an anti-linear operator is a conjugation if and only if it is both isometric and involutive. This note suggests us to characterize all isometric as well as all involutive anti-linear weighted composition operators on $\mathcal{F}^{2}(\mathbb{C})$. Combining these characterizations yields the structure of an anti-linear weighted composition operator, when it is a conjugation on $\mathcal{F}^{2}(\mathbb{C})$.

Having constructed conjugations on $\mathcal{F}^{2}(\mathbb{C})$, we characterize bounded weighted composition operators, which are complex symmetric with such conjugations. Several spectral properties of weighted composition operators are investigated. 


\subsection{Anti-linear weighted composition operators}

For given entire functions $\xi$ and $\eta$, we define the operator $\mathcal{A}_{\xi, \eta}$ by

$$
\left(\mathcal{A}_{\xi, \eta} f\right)(z)=\xi(z) \overline{f(\overline{\eta(z)})} .
$$

We prove the following observation.

Proposition 2.1.1. The operator $\mathcal{A}_{\xi, \eta}$ is anti-linear.

Proof. For every $f, g \in \mathcal{F}^{2}(\mathbb{C})$, and $\alpha, \beta \in \mathbb{C}$, we have

$$
\begin{aligned}
\mathcal{A}_{\xi, \eta}(\alpha f+\beta g)(z) & =\xi(z) \overline{(\alpha f+\beta g)(\overline{\eta(z)})}=\xi(z) \overline{(\alpha f)(\overline{\eta(z)})}+\xi(z) \overline{(\beta g)(\overline{\eta(z)})} \\
& =\xi(z) \overline{\alpha f(\overline{\eta(z)})}+\xi(z) \bar{\beta} \overline{g(\overline{\eta(z)})}=\bar{\alpha}\left(\mathcal{A}_{\xi, \eta} f\right)(z)+\bar{\beta}\left(\mathcal{A}_{\xi, \eta} g\right)(z) .
\end{aligned}
$$

The operator $\mathcal{A}_{\xi, \eta}$ is called the anti-linear weighted composition operator, induced by the symbols $\xi$ and $\eta$. In particular, with $\xi \equiv \mathbf{1}$ and $\eta(z)=z$, we have the following result.

Lemma 2.1.2. The operator $\mathcal{J} f(z)=\overline{f(\bar{z})}$ is a conjugation on $\mathcal{F}^{2}(\mathbb{C})$.

Proof. For every $f(z)=\sum_{m \geq 0} f_{m} z^{m} \in \mathcal{F}^{2}(\mathbb{C})$, we have

$$
(\mathcal{J} f)(z)=\overline{f(\bar{z})}=\overline{\sum_{m \geq 0} f_{m} \bar{z}^{m}}=\sum_{m \geq 0} \overline{f_{m}} z^{m},
$$

which implies, since $\left(z^{m}\right)$ is an orthogonal basis in $\mathcal{F}^{2}(\mathbb{C})$, that

$$
\|\mathcal{J} f\|^{2}=\sum_{m \geq 0}\left|\overline{f_{m}}\right|^{2}\left\|z^{m}\right\|^{2}=\|f\|^{2} .
$$

The last equality shows that $\mathcal{J} f \in \mathcal{F}^{2}(\mathbb{C})$, and hence, $\mathcal{J}$ is isometric.

On the other hand, the operator $\mathcal{J}$ is involutive, because

$$
\mathcal{J}^{2} f(z)=\mathcal{J}\left(\sum_{m \geq 0} \overline{f_{m}} z^{m}\right)(z)=\sum_{m \geq 0} f_{m} z^{m}, \quad \forall z \in \mathbb{C} .
$$

Thus, $\mathcal{J} f(z)=\overline{f(\bar{z})}$ is a conjugation on $\mathcal{F}^{2}(\mathbb{C})$. 
We note that the operator $\mathcal{J}$ is a conjugation on Hardy spaces in the unit disk (see [7], [15]).

A question to ask is: is there any relationship between a linear operator $W_{\xi, \eta}$ and anti-linear operator $\mathcal{A}_{\xi, \eta}$ ? The answer is affirmative, and more than that, as we can see below, the conjugation $\mathcal{J}$ plays an essential role in the relationship.

Proposition 2.1.3. We always have $\mathcal{A}_{\xi, \eta}=W_{\xi, \eta} \mathcal{J}$, or equivalently $W_{\xi, \eta}=\mathcal{A}_{\xi, \eta} \mathcal{J}$.

Proof. Take arbitrarily $f \in \mathcal{F}^{2}(\mathbb{C})$. Since $\mathcal{J} f(z)=\overline{f(\bar{z})}$, we have

$$
\left(W_{\xi, \eta} \mathcal{J}\right) f(z)=\xi(z) \mathcal{J} f(\eta(z))=\xi(z) \overline{f(\overline{\eta(z)})}=\left(\mathcal{A}_{\xi, \eta} f\right)(z), \quad \forall z \in \mathbb{C}
$$

Furthermore, the formula $\mathcal{A}_{\xi, \eta}=W_{\xi, \eta} \mathcal{J}$ is equivalent to $W_{\xi, \eta}=\mathcal{A}_{\xi, \eta} \mathcal{J}$, because $\mathcal{J}$ is involutive.

Thanks to Proposition 2.1.3, we can show that $W_{\xi, \eta}$ and $\mathcal{A}_{\xi, \eta}$ have several common important properties. Namely, we have the following result.

Theorem 2.1.4. Let $\xi, \eta$ be two entire functions with $\xi \not \equiv \mathbf{0}$. The following statements hold:

1. $\mathcal{A}_{\xi, \eta}$ is bounded on $\mathcal{F}^{2}(\mathbb{C})$ if and only if so is $W_{\xi, \eta}$. In this case, $\left\|\mathcal{A}_{\xi, \eta}\right\|=$ $\left\|W_{\xi, \eta}\right\|$.

2. $\mathcal{A}_{\xi, \eta}$ is isometric on $\mathcal{F}^{2}(\mathbb{C})$ if and only if so is $W_{\xi, \eta}$.

Proof. Arbitrarily take $f \in \mathcal{F}^{2}(\mathbb{C})$.

(1) - Suppose that $\mathcal{A}_{\xi, \eta}$ is bounded on $\mathcal{F}^{2}(\mathbb{C})$. By Proposition 2.1.3, we have $W_{\xi, \eta}=\mathcal{A}_{\xi, \eta} \mathcal{J}$, which gives

$$
\begin{aligned}
\left\|W_{\xi, \eta} f\right\| & =\left\|\mathcal{A}_{\xi, \eta} \mathcal{J} f\right\| \leq\left\|\mathcal{A}_{\xi, \eta}\right\| \cdot\|\mathcal{J} f\| \quad \text { (as } \mathcal{A}_{\xi, \eta} \text { is bounded) } \\
& =\left\|\mathcal{A}_{\xi, \eta}\right\| \cdot\|f\| \quad \text { (as } \mathcal{J} \text { is isometric). }
\end{aligned}
$$


Thus, $W_{\xi, \eta}$ is bounded and $\left\|W_{\xi, \eta}\right\| \leq\left\|\mathcal{A}_{\xi, \eta}\right\|$.

- Conversely, suppose that $W_{\xi, \eta}$ is bounded on $\mathcal{F}^{2}(\mathbb{C})$. By Proposition 2.1.3. we have $\mathcal{A}_{\xi, \eta}=W_{\xi, \eta} \mathcal{J}$, which provides

$$
\begin{aligned}
\left\|\mathcal{A}_{\xi, \eta} f\right\| & \left.=\left\|W_{\xi, \eta} \mathcal{J} f\right\| \leq\left\|W_{\xi, \eta}\right\| \cdot\|\mathcal{J} f\| \quad \text { (as } W_{\xi, \eta} \text { is bounded }\right) \\
& =\left\|W_{\xi, \eta}\right\| \cdot\|f\| \quad(\text { as } \mathcal{J} \text { is isometric }) .
\end{aligned}
$$

Thus, $\mathcal{A}_{\xi, \eta}$ is bounded and $\left\|\mathcal{A}_{\xi, \eta}\right\| \leq\left\|W_{\xi, \eta}\right\|$.

(2) • Suppose that $\mathcal{A}_{\xi, \eta}$ is isometric on $\mathcal{F}^{2}(\mathbb{C})$. Again by Proposition 2.1.3, we have $W_{\xi, \eta}=\mathcal{A}_{\xi, \eta} \mathcal{J}$, which gives

$$
\begin{aligned}
\left\|W_{\xi, \eta} f\right\| & \left.=\left\|\mathcal{A}_{\xi, \eta} \mathcal{J} f\right\|=\|\mathcal{J} f\| \quad \text { (as } \mathcal{A}_{\xi, \eta} \text { is isometric }\right) \\
& =\|f\| \quad(\text { as } \mathcal{J} \text { is isometric }) .
\end{aligned}
$$

Thus, $W_{\xi, \eta}$ is isometric.

- Conversely, suppose that $W_{\xi, \eta}$ is isometric on $\mathcal{F}^{2}(\mathbb{C})$. By Proposition 2.1.3. we have $\mathcal{A}_{\xi, \eta}=W_{\xi, \eta} \mathcal{J}$, which provides

$$
\begin{aligned}
\left\|\mathcal{A}_{\xi, \eta} f\right\| & =\left\|W_{\xi, \eta} \mathcal{J} f\right\|=\|\mathcal{J} f\| \quad \text { (as } W_{\xi, \eta} \text { is isometric) } \\
& =\|f\| \quad(\text { as } \mathcal{J} \text { is isometric }) .
\end{aligned}
$$

Thus, $\mathcal{A}_{\xi, \eta}$ is isometric.

In the sequel, we investigate a problem of whether anti-linear weighted composition operators can be conjugations on the Fock space $\mathcal{F}^{2}(\mathbb{C})$. Note that Definition 1.1.5 suggests us to identify all isometric as well as all involutive anti-linear weighted composition operators.

The following identity in a general Hilbert space $\mathcal{H}$ (including $\mathbb{C}^{n}$ ) is useful in later computations:

$$
\|\mathbf{a}+\mathbf{b}\|^{2}=\|\mathbf{a}\|^{2}+\|\mathbf{b}\|^{2}+2 \operatorname{Re}\langle\mathbf{a}, \mathbf{b}\rangle, \quad \forall \mathbf{a}, \mathbf{b} \in \mathcal{H}
$$




\subsubsection{Boundedness, isometry}

First, we should know when the operator $\mathcal{A}_{\xi, \eta}$ acts from the Fock space $\mathcal{F}^{2}(\mathbb{C})$ into itself. Since by (1.7), a point evaluation is bounded on $\mathcal{F}^{2}(\mathbb{C})$, this space $\mathcal{F}^{2}(\mathbb{C})$ is a functional Hilbert space, and so by the Closed Graph Theorem, $\mathcal{A}_{\xi, \eta}$ is $\mathcal{F}^{2}(\mathbb{C})$-invariant if and only if it is bounded on $\mathcal{F}^{2}(\mathbb{C})$.

- For the case when $\eta$ is a non-constant function, we have the following result.

Proposition 2.1.5. Let $\eta \not \equiv$ const, $\xi \not \equiv \mathbf{0}$ be two entire functions. The following assertions are equivalent:

1. The linear weighted composition operator $W_{\xi, \eta}: \mathcal{F}^{2}(\mathbb{C}) \rightarrow \mathcal{F}^{2}(\mathbb{C})$ is bounded on $\mathcal{F}^{2}(\mathbb{C})$.

2. The anti-linear weighted composition operator $\mathcal{A}_{\xi, \eta}: \mathcal{F}^{2}(\mathbb{C}) \rightarrow \mathcal{F}^{2}(\mathbb{C})$ is bounded on $\mathcal{F}^{2}(\mathbb{C})$.

3. The symbols $\xi, \eta$ satisfy the condition $M(\xi, \eta)<\infty$.

Moreover, in this case, $\eta(z)=a z+b$ with $0<|a| \leq 1$, and

$$
\sqrt{M_{z}(\xi, \eta)} \leq\left\|\mathcal{A}_{\xi, \eta}\right\|=\left\|W_{\xi, \eta}\right\| \leq \frac{\sqrt{M(\xi, \eta)}}{|a|}, \quad \forall z \in \mathbb{C} .
$$

Proof. The equivalence $(1) \Longleftrightarrow(2)$ is followed from Theorem 2.1.4. Now we prove that $(2) \Longleftrightarrow(3)$.

- Suppose that (2) holds. By Theorem 2.1.4, we have $\left\|\mathcal{A}_{\xi, \eta}\right\|=\left\|W_{\xi, \eta}\right\|$, and moreover,

$$
\left\|\mathcal{A}_{\xi, \eta}(g)\right\| \leq\left\|\mathcal{A}_{\xi, \eta}\right\| \cdot\|g\|, \forall g \in \mathcal{F}^{2}(\mathbb{C})
$$

Then for every $z, u \in \mathbb{C}$, we have

$$
\begin{aligned}
|\xi(z)| \cdot\left|e^{u \eta(z)-\frac{|u|^{2}}{2}}\right| e^{-\frac{|z|^{2}}{2}} & =\left|\left(\mathcal{A}_{\xi, \eta}\left(k_{u}\right)\right)(z)\right| e^{-\frac{|z|^{2}}{2}} \leq\left\|\mathcal{A}_{\xi, \eta}\left(k_{u}\right)\right\| \quad(\text { by }(1.7)) \\
& \leq\left\|\mathcal{A}_{\xi, \eta}\right\| \cdot\left\|k_{u}\right\|=\left\|\mathcal{A}_{\xi, \eta}\right\| \quad \text { (since } k_{u} \text { is a unit vector). }
\end{aligned}
$$


In particular, letting $u=\overline{\eta(z)}$, the last inequality becomes $\sqrt{M_{z}(\xi, \eta)}$, and hence, the first estimate of $(2.2)$ is true. Taking the supremum by $z$ over $\mathbb{C}$ yields (3).

- Conversely, suppose that $M(\xi, \eta)<\infty$. By Lemma 1.4.2, $\eta(z)=a z+b$ with $|a| \leq 1$, and furthermore, $0<|a| \leq 1$ (since $\eta$ is a non-constant function).

For every $f \in \mathcal{F}^{2}(\mathbb{C})$, we have

$$
\begin{aligned}
\left\|\mathcal{A}_{\xi, \eta}(f)\right\| & =\left(\frac{1}{\pi} \int_{\mathbb{C}} M_{z}(\xi, \eta)|\mathcal{J} f(\eta(z))|^{2} e^{-|\eta(z)|^{2}} d V(z)\right)^{1 / 2} \\
& \leq \sqrt{M(\xi, \eta)}\left(\frac{1}{\pi} \int_{\mathbb{C}}|\mathcal{J} f(\eta(z))|^{2} e^{-|\eta(z)|^{2}} d V(z)\right)^{1 / 2} \\
& \left.=\frac{\sqrt{M(\xi, \eta)}}{|a|}\|\mathcal{J} f\| \quad \text { (by changing the variables } u=\eta(z)\right),
\end{aligned}
$$

which, due to the isometry of $\mathcal{J}$, gives 2.2 .

- For the case when $\eta$ is a constant function, our characterization is simpler.

Proposition 2.1.6. Let $\xi \not \equiv \mathbf{0}$ be an entire function, and $\eta \equiv b$ a constant function. The following assertions are equivalent:

1. The linear weighted composition operator $W_{\xi, \eta}: \mathcal{F}^{2}(\mathbb{C}) \rightarrow \mathcal{F}^{2}(\mathbb{C})$ is bounded on $\mathcal{F}^{2}(\mathbb{C})$.

2. The anti-linear weighted composition operator $\mathcal{A}_{\xi, \eta}: \mathcal{F}^{2}(\mathbb{C}) \rightarrow \mathcal{F}^{2}(\mathbb{C})$ is bounded on $\mathcal{F}^{2}(\mathbb{C})$.

3. The function $\xi$ belongs to $\mathcal{F}^{2}(\mathbb{C})$.

Proof. It is similar to those of Proposition 2.1.5. The equivalence (1) $\Longleftrightarrow$ holds by Theorem 2.1.4. Now we prove that $(2) \Longleftrightarrow(3)$.

- Suppose that $\mathcal{A}_{\xi, \eta}$ is bounded. Then $\xi=\mathcal{A}_{\xi, \eta}(\mathbf{1}) \in \mathcal{F}^{2}(\mathbb{C})$.

- Conversely, suppose $\xi \in \mathcal{F}^{2}(\mathbb{C})$. For every $f \in \mathcal{F}^{2}(\mathbb{C})$, by 1.7$)$, we have

$$
\left\|\mathcal{A}_{\xi, \eta}(f)\right\|=\|\xi\| \cdot|f(b)| \leq\|\xi\| e^{\frac{|b|^{2}}{2}}\|f\|
$$


Thus, $\mathcal{A}_{\xi, \eta}$ is bounded, and moreover, its norm satisfies

$$
\left\|\mathcal{A}_{\xi, \eta}\right\| \leq\|\xi\| e^{\frac{|b|^{2}}{2}}
$$

- Both cases can be combined in the following statement.

Theorem 2.1.7. Let $\xi, \eta$ be two entire functions with $\xi \not \equiv \mathbf{0}$. The following assertions are equivalent.

1. The linear weighted composition operator $W_{\xi, \eta}: \mathcal{F}^{2}(\mathbb{C}) \rightarrow \mathcal{F}^{2}(\mathbb{C})$ is $\mathcal{F}^{2}(\mathbb{C})$ invariant (or bounded on $\mathcal{F}^{2}(\mathbb{C})$ ).

2. The anti-linear weighted composition operator $\mathcal{A}_{\xi, \eta}: \mathcal{F}^{2}(\mathbb{C}) \rightarrow \mathcal{F}^{2}(\mathbb{C})$ is $\mathcal{F}^{2}(\mathbb{C})$-invariant (or bounded on $\mathcal{F}^{2}(\mathbb{C})$ ).

3. The symbols satisfy $\xi \in \mathcal{F}^{2}(\mathbb{C})$ and $M(\xi, \eta)<\infty$.

In this case, $\eta(z)=a z+b$ with $|a| \leq 1$.

Proof. The equivalence $(1) \Longleftrightarrow(3)$ holds by Proposition 1.4.1, while the equivalence $(1) \Longleftrightarrow(2)$ holds by Theorem 2.1 .4 .

Concerning the statement (3) of the result above, a question arises: is there any relationship between the condition $\xi \in \mathcal{F}^{2}(\mathbb{C})$ and the condition $M(\xi, \eta)<\infty$ ? The propositions below gives us the answer.

Proposition 2.1.8. Let $\eta \equiv b$ a constant function, and $\xi$ an entire function. If $\xi \in \mathcal{F}^{2}(\mathbb{C})$, then $M(\xi, \eta)<\infty$.

Proof. Since $\eta(z)=b$ and $\xi \in \mathcal{F}^{2}(\mathbb{C})$, we have by (1.7)

$$
M_{z}(\xi, \eta)=|\xi(z)|^{2} e^{|b|^{2}-|z|^{2}}=e^{|b|^{2}}|\xi(z)|^{2} e^{-|z|^{2}} \leq e^{|b|^{2}}\|\xi\|^{2}, \quad \forall z \in \mathbb{C} .
$$

Then taking the supremum by $z$ over $\mathbb{C}$ yields the desired conclusion. 
Proposition 2.1.9. Let $\eta \not \equiv$ const, $\xi \not \equiv \mathbf{0}$ be two entire functions. If $M(\xi, \eta)<\infty$, then $\xi \in \mathcal{F}^{2}(\mathbb{C})$.

Proof. Suppose that $M(\xi, \eta)<\infty$. By Lemma 1.4.2, $\eta(z)=a z+b$ with $|a| \leq 1$, and furthermore, $0<|a| \leq 1$ (since $\eta$ is a non-constant function).

Since $M_{z}(\xi, \eta) \leq M(\xi, \eta)$ for every $z \in \mathbb{C}$, we have

$$
\begin{aligned}
\|\xi\|^{2} & =\frac{1}{\pi} \int_{\mathbb{C}} M_{z}(\xi, \eta) e^{-|a z+b|^{2}} d V(z) \\
& \leq \frac{M(\xi, \eta)}{\pi} \int_{\mathbb{C}} e^{-|a z+b|^{2}} d V(z) .
\end{aligned}
$$

Changing the variables $u=a z+b$ gives

$$
\begin{aligned}
\|\xi\|^{2} & \leq \frac{M(\xi, \eta)}{|a|^{2} \pi} \int_{\mathbb{C}} e^{-|u|^{2}} d V(u) \\
& =\frac{M(\xi, \eta)}{|a|^{2} \pi} \lim _{R \rightarrow \infty} \int_{|u| \leq R} e^{-|u|^{2}} d V(u) .
\end{aligned}
$$

Using polar coordinates $\left(u=r e^{i \theta}\right)$, we see that

$$
\begin{aligned}
\|\xi\|^{2} & \leq \frac{M(\xi, \eta)}{|a|^{2} \pi} \lim _{R \rightarrow \infty} \int_{0}^{2 \pi} d \theta \int_{0}^{R} r e^{-r^{2}} d r \\
& =\frac{2 M(\xi, \eta)}{|a|^{2}} \lim _{R \rightarrow \infty} \int_{0}^{R} r e^{-r^{2}} d r \\
& =\frac{M(\xi, \eta)}{|a|^{2}} \lim _{R \rightarrow \infty} \int_{0}^{R^{2}} e^{-t} d t=\frac{M(\xi, \eta)}{|a|^{2}}<\infty .
\end{aligned}
$$

Note that Propositions 2.1.5 2.1.6 and Propositions 2.1.8 2.1.9 can offer a direct proof for the equivalence $(2) \Longleftrightarrow(3)$ of Theorem 2.1.7.

Next, we give two examples in order to show that the inverse directions of Proposition 2.1.8 as well as Proposition 2.1.9 are not true. 
Example 2.1.10. The function $\xi(z)=e^{z^{2} / 2}$ does not belong to $\mathcal{F}^{2}(\mathbb{C})$ by $[13$, Theorem 1.1], but

$$
\begin{aligned}
M_{z}(\xi, \mathbf{0}) & =|\xi(z)|^{2} e^{-|z|^{2}}=e^{\operatorname{Re} z^{2}-\left|z^{2}\right|} \\
& =e^{\operatorname{Re} z^{2}-\sqrt{\left(\operatorname{Re} z^{2}\right)^{2}+\left(\operatorname{Im} z^{2}\right)^{2}}} \leq 1, \quad \forall z \in \mathbb{C} .
\end{aligned}
$$

Example 2.1.11. Let $\xi(z)=e^{z} \in \mathcal{F}^{2}(\mathbb{C}), \eta(z)=z$. Then

$$
M_{z}(\xi, \eta)=|\xi(z)|^{2} e^{|z|^{2}-|z|^{2}}=|\xi(z)|^{2}=e^{2 \operatorname{Re} z}
$$

is unbounded.

As it will be seen later for complex symmetric weighted composition operators, the symbol $\xi$ has an exponential form, while $\eta$ is affine. Thus, it is essential to precise conditions in Proposition 1.4.1. It will be important steps toward studying a complex symmetry of bounded linear weighted composition operators on the Fock space $\mathcal{F}^{2}(\mathbb{C})$.

The following lemma shows that the expression $M_{z}(\xi, \eta)$ can be indicated explicitly in the case when the symbol $\xi$ has an exponential form and $\eta$ is affine.

Lemma 2.1.12. Let $\eta(z)=a z+b$ and $\xi(z)=c e^{d z}$.

1. There is the identity

$$
M_{z}(\xi, \eta)=|c|^{2} e^{|b|^{2}} e^{-\left(1-|a|^{2}\right) x^{2}+2 x \operatorname{Re}(d+a \bar{b})-\left(1-|a|^{2}\right) y^{2}-2 y \operatorname{Im}(d+a \bar{b})},
$$

where $x=\operatorname{Re} z$ and $y=\operatorname{Im} z$.

2. The following inequality holds.

$$
M_{z}(\xi, \eta) \leq|c|^{2} e^{|b|^{2}} e^{-\left(1-|a|^{2}\right)|z|^{2}+2(|a b|+|d|)|z|}, \forall z \in \mathbb{C} .
$$


Proof. (1) We have

$$
\begin{aligned}
M_{z}(\xi, \eta) & =\left|c e^{d z}\right|^{2} e^{|a z+b|^{2}-|z|^{2}}=|c|^{2} e^{2 \operatorname{Re}(d z)} e^{|a z+b|^{2}-|z|^{2}} \\
& =|c|^{2} e^{2 \operatorname{Re}(d z)+|a z|^{2}+|b|^{2}+2 \operatorname{Re}(a \bar{b} z)-|z|^{2}} \\
& =|c|^{2} e^{|a z|^{2}+|b|^{2}+2 \operatorname{Re}(z(d+a \bar{b}))-|z|^{2}} \\
& =|c|^{2} e^{|b|^{2}} e^{-\left(1-|a|^{2}\right) x^{2}+2 x \operatorname{Re}(d+a \bar{b})-\left(1-|a|^{2}\right) y^{2}-2 y \operatorname{Im}(d+a \bar{b})} .
\end{aligned}
$$

(2) Applying the Cauchy-Schwarz inequality, we have

$$
\begin{aligned}
x \operatorname{Re}(d+a \bar{b})-y \operatorname{Im}(d+a \bar{b}) & \leq \sqrt{x^{2}+y^{2}} \cdot \sqrt{[\operatorname{Re}(d+a \bar{b})]^{2}+[\operatorname{Im}(d+a \bar{b})]^{2}} \\
& =|z| \cdot|d+a \bar{b}| \leq|z|(|d|+|a b|),
\end{aligned}
$$

which gives 2.4.

In the case when $\eta(z)=a z+b$ and $\xi(z)=c e^{d z}$, characterizations for boundedness and compactness are simplified as follows.

Proposition 2.1.13. Let $\eta(z)=a z+b, \xi(z)=c e^{d z}$, where $a, b, c$, and $d$ are complex constants with $c \neq 0$. The following statements are equivalent:

1. The linear weighted composition operator $W_{\xi, \eta}$ is bounded on $\mathcal{F}^{2}(\mathbb{C})$.

2. The anti-linear weighted composition operator $\mathcal{A}_{\xi, \eta}$ is bounded on $\mathcal{F}^{2}(\mathbb{C})$.

3. One of the following conditions holds.

(a) either $|a|<1$

(b) or $|a|=1, d+a \bar{b}=0$.

In the case (a),

$$
M(\xi, \eta)=|c|^{2} e^{|b|^{2}+\frac{\left(|a b|+\left.|a|\right|^{2}\right.}{1-|a|^{2}}}
$$

In the case (b),

$$
M_{z}(\xi, \eta)=|c|^{2} e^{|b|^{2}}, \forall z \in \mathbb{C}
$$


Proof. The equivalence $(1) \Longleftrightarrow(2)$ holds by Theorem 2.1.4. Now we prove that (1) $\Longleftrightarrow(3)$.

Clearly, $\xi \in \mathcal{F}^{2}(\mathbb{C})$. Note that if $a=0$, then by Proposition 2.1.6. $W_{\xi, \eta}$ is always bounded on $\mathcal{F}^{2}(\mathbb{C})$. Suppose $a \neq 0$. In this case, by Proposition 2.1.5, $W_{\xi, \eta}$ is bounded if and only if

$$
M(\xi, \eta)<\infty
$$

The expression $M(\xi, \eta)$ is always finite in the case that $|a|<1$, because

$$
\begin{aligned}
M(\xi, \eta) & =\sup _{z \in \mathbb{C}} M_{z}(\xi, \eta)=|c|^{2} e^{|b|^{2}} \sup _{x, y \in \mathbb{R}} e^{-\left(1-|a|^{2}\right) x^{2}+2 x \operatorname{Re}(d+a \bar{b})-\left(1-|a|^{2}\right) y^{2}-2 y \operatorname{Im}(d+a \bar{b})} \\
& =|c|^{2} e^{|b|^{2}} \sup _{x \in \mathbb{R}} e^{-\left(1-|a|^{2}\right) x^{2}+2 x \operatorname{Re}(d+a \bar{b})} \cdot \sup _{y \in \mathbb{R}} e^{-\left(1-|a|^{2}\right) y^{2}-2 y \operatorname{Im}(d+a \bar{b})} \\
& =|c|^{2} e^{|b|^{2}} e^{\frac{(\operatorname{Re}(d+a \bar{b}))^{2}+(\operatorname{Im}(d+a \bar{b}))^{2}}{1-|a|^{2}}}=|c|^{2} e^{|b|^{2}} e^{\frac{|d+a \bar{b}|^{2}}{1-|a|^{2}}} .
\end{aligned}
$$

If $|a|=1$, then the supremum of $(2.5)$ is finite if and only if $\operatorname{Re}(d+a \bar{b})=\operatorname{Im}(d+$ $a \bar{b})=0$, or equivalently if and only if $d+a \bar{b}=0$. Hence,

$$
M_{z}(\xi, \eta)=|c|^{2} e^{|b|^{2}}, \forall z \in \mathbb{C} \quad \Longrightarrow \quad M(\xi, \eta)=|c|^{2} e^{|b|^{2}}
$$

In what follows, we always assume that $\xi$ and $\eta$ are entire functions with $\eta \not \equiv$ const and $\xi \not \equiv \mathbf{0}$, for which $M(\xi, \eta)<\infty$, that is, $\mathcal{A}_{\xi, \eta}$ is bounded on $\mathcal{F}^{2}(\mathbb{C})$.

We study an isometry of anti-linear weighted composition operators.

Proposition 2.1.14. The following assertions are equivalent:

1. The linear weighted composition operator $W_{\xi, \eta}$ is isometric on $\mathcal{F}^{2}(\mathbb{C})$.

2. The anti-linear weighted composition operator $\mathcal{A}_{\xi, \eta}$ is isometric on $\mathcal{F}^{2}(\mathbb{C})$.

3. The symbols satisfy

$$
\eta(z)=a z+b, \quad \xi(z)=c e^{-a \bar{b} z}
$$


where $a, b$, and $c$ are complex constants satisfying

$$
|a|=1, \quad|c|^{2} e^{|b|^{2}}=1 .
$$

The first proof. The equivalence $(1) \Longleftrightarrow(2)$ holds true by Theorem 2.1.4, while the equivalence of (1) and (3) was proved in Proposition 1.4.7.

For the equivalence of (2) and (3), we can give a direct proof without using Theorem 2.1.4. This proof needs the following lemma.

Lemma 2.1.15. For every $b \in \mathbb{C}$, the anti-linear operator $V_{b}: \mathcal{F}^{2}(\mathbb{C}) \rightarrow \mathcal{F}^{2}(\mathbb{C})$, defined by

$$
V_{b} f(z)=k_{-b}(z) \overline{f(\overline{z+b})},
$$

is isometric on $\mathcal{F}^{2}(\mathbb{C})$.

Proof. We note the following identity

$$
\left|k_{-\beta}(\alpha-\beta)\right|^{2} e^{-|\alpha-\beta|^{2}}=e^{-|\alpha|^{2}}, \quad \forall \alpha, \beta \in \mathbb{C} .
$$

Indeed, we have

$$
\begin{aligned}
\left|k_{-\beta}(\alpha-\beta)\right|^{2} e^{-|\alpha-\beta|^{2}} & =\left|k_{-\beta}(\alpha-\beta)\right|^{2} \cdot e^{-|\alpha|^{2}-|\beta|^{2}+2 \operatorname{Re}(\bar{\beta} \alpha)} \quad(\text { by }(2.1)) \\
& =\left|e^{-2 \bar{\beta} \alpha+|\beta|^{2}}\right| \cdot e^{-|\alpha|^{2}-|\beta|^{2}+2 \operatorname{Re}(\bar{\beta} \alpha)}=e^{-|\alpha|^{2}} .
\end{aligned}
$$

For every $f \in \mathcal{F}^{2}(\mathbb{C})$, by changing the variables $u=z+b$, we have

$$
\begin{aligned}
\left\|V_{b} f\right\|^{2} & =\frac{1}{\pi} \int_{\mathbb{C}}\left|k_{-b}(z)\right|^{2} \cdot|\mathcal{J} f(z+b)|^{2} e^{-|z|^{2}} d V(z) \\
& =\frac{1}{\pi} \int_{\mathbb{C}}\left|k_{-b}(u-b)\right|^{2}|\mathcal{J} f(u)|^{2} e^{-|u-b|^{2}} d V(u) \\
& =\frac{1}{\pi} \int_{\mathbb{C}}|\mathcal{J} f(u)|^{2} e^{-|u|^{2}} d V(u) \quad(\text { by }(2.9)) \\
& =\|\mathcal{J} f\|^{2}=\|f\|^{2} .
\end{aligned}
$$


Thanks to Lemma 2.1.15, we give the second proof for the equivalence of (2) and (3).

The second proof. • Suppose that $\mathcal{A}_{\xi, \eta}$ is isometric on $\mathcal{F}^{2}(\mathbb{C})$. By Proposition 1.4.1. $\eta(z)=a z+b$ for some constants $a$ and $b$ with $|a| \leq 1$. For every $f \in \mathcal{F}^{2}(\mathbb{C})$, we have

$$
V_{-\bar{b}} f(z)=k_{\bar{b}}(z) \overline{f(\bar{z}-b)},
$$

and hence $V_{-\bar{b}} f(\overline{\eta(z)})=k_{\bar{b}}(\overline{a z+b}) \overline{f(a z)}$. From this, it follows that

$$
\begin{aligned}
\mathcal{A}_{\xi, \eta} V_{-\bar{b}} f(z) & =\xi(z) \overline{V_{-\bar{b}} f(\overline{\eta(z)})}=\xi(z) e^{a \bar{b} z+\frac{|b|^{2}}{2}} f(a z) \\
& =\xi(z) k_{b}(a z+b) f(a z), \quad z \in \mathbb{C} .
\end{aligned}
$$

Setting $\zeta(z)=\xi(z) k_{b}(a z+b)$ and $\chi(z)=a z$, we see that

$$
\mathcal{A}_{\xi, \eta} V_{-\bar{b}}=W_{\zeta, \chi}
$$

which is the linear weighted composition operator on $\mathcal{F}^{2}(\mathbb{C})$, induced by symbols $\zeta$ and $\chi$.

Since $\mathcal{A}_{\xi, \eta}$ is isometric, by Lemma 2.1.15, we have

$$
\left\|\mathcal{A}_{\xi, \eta} V_{-\bar{b}} f\right\|=\left\|V_{-\bar{b}} f\right\|=\|f\|, \quad \forall f \in \mathcal{F}^{2}(\mathbb{C}),
$$

and so, $\mathcal{A}_{\xi, \eta} V_{-\bar{b}}$ is isometric.

Furthermore, since $W_{\zeta, \chi}$ is isometric on $\mathcal{F}^{2}(\mathbb{C})$, by Proposition $1.4 .7,|a|=1$ and $\zeta$ must be a constant of modulus 1. Consequently, denoting $\theta=\xi(z) k_{b}(a z+b)$, we have $|\theta|=1$ and $\xi(z)=\theta e^{-|b|^{2} / 2} e^{-a \bar{b} z}$. Then for $c=\theta e^{-|b|^{2} / 2}$, we get $\theta=c e^{|b|^{2} / 2}$. Since $|\theta|=1$, we have $\left|c e^{|b|^{2} / 2}\right|=1$. Thus, (2.7) and (2.8) hold.

- Conversely, suppose that $\eta$, $\xi$ satisfy 2.7 and (2.8). For every $f \in \mathcal{F}^{2}(\mathbb{C})$, by (2.7), we have

$$
\left\|\mathcal{A}_{\xi, \eta} f\right\|^{2}=\frac{1}{\pi} \int_{\mathbb{C}}\left|c e^{-a \bar{b} z}\right|^{2}|\mathcal{J} f(a z+b)|^{2} e^{-|z|^{2}} d V(z) .
$$


Furthermore, since $|a|=1$, the changing the variables $u=a z$ yields

$$
\begin{aligned}
\left\|\mathcal{A}_{\xi, \eta} f\right\|^{2} & =\frac{1}{\pi} \int_{\mathbb{C}}\left|c e^{-\bar{b} u}\right|^{2}|\mathcal{J} f(u+b)|^{2} e^{-|u|^{2}} d V(u) \\
& =\frac{1}{\pi} \int_{\mathbb{C}}|c|^{2} e^{|b|^{2}}\left|e^{-\bar{b} u-|b|^{2} / 2}\right|^{2}|\mathcal{J} f(u+b)|^{2} e^{-|u|^{2}} d V(u) \\
& =\frac{1}{\pi} \int_{\mathbb{C}}\left|k_{-b}(u)\right|^{2}|\mathcal{J} f(u+b)|^{2} e^{-|u|^{2}} d V(u) \\
& =\left\|V_{b} f\right\|^{2}=\|f\|^{2} .
\end{aligned}
$$

The proof is complete.

\subsubsection{Involution}

In this subsection, we characterize bounded anti-linear weighted composition operators, which are involutive on the Fock space $\mathcal{F}^{2}(\mathbb{C})$.

Proposition 2.1.16. Suppose that the anti-linear weighted composition operator $\mathcal{A}_{\xi, \eta}: \mathcal{F}^{2}(\mathbb{C}) \rightarrow \mathcal{F}^{2}(\mathbb{C})$ is bounded. Then $\mathcal{A}_{\xi, \eta}$ is involutive on $\mathcal{F}^{2}(\mathbb{C})$ if and only if the symbols are of forms (2.7), i.e.

$$
\eta(z)=a z+b, \quad \xi(z)=c e^{-a \bar{b} z}
$$

where $a, b$, and $c$ are complex constants satisfying condition (2.8) with $\bar{a} b+\bar{b}=0$, i.e.

$$
|a|=1, \quad \bar{a} b+\bar{b}=0, \quad|c|^{2} e^{|b|^{2}}=1 .
$$

Proof. Since $\mathcal{A}_{\xi, \eta}$ is bounded on $\mathcal{F}^{2}(\mathbb{C})$, by Theorem 2.1.7, $\eta(z)=a z+b$ with $|a| \leq 1$, and hence,

$$
\begin{aligned}
\overline{\eta(\overline{\eta(z)})} & =\overline{a \overline{\eta(z)}+b}=\bar{a} \eta(z)+\bar{b} \\
& =\bar{a}(a z+b)+\bar{b} \\
& =|a|^{2} z+\bar{a} b+\bar{b}, \quad \forall z \in \mathbb{C} .
\end{aligned}
$$


Clearly, $\mathcal{A}_{\xi, \eta}$ is involutive if and only if

$$
\mathcal{A}_{\xi, \eta}^{2} f(z)=\xi(z) \overline{\xi(\overline{\eta(z)})} f(\overline{\eta(\overline{\eta(z)})})=f(z), \forall f \in \mathcal{F}^{2}(\mathbb{C})
$$

- Suppose that $\mathcal{A}_{\xi, \eta}$ is involutive, that is 2.12 holds. In particular, with $f \equiv \mathbf{1}$, we obtain

$$
\xi(z) \overline{\xi(\overline{\eta(z)})}=1
$$

and hence, equation 2.12 is reduced to

$$
f(\overline{\eta(\overline{\eta(z)})})=f(z), \forall f \in \mathcal{F}^{2}(\mathbb{C}) .
$$

Now taking $f(z) \equiv z$ in 2.14 , we get

$$
\overline{\eta(\overline{\eta(z)})}=z, \forall z \in \mathbb{C}
$$

which implies, by (2.11), that $|a|=1$ and $\bar{a} b+\bar{b}=0$.

Since $|a|=1$, by Lemma 1.4 .2 , we have $\xi(z)=c e^{-a \bar{b} z}, c \in \mathbb{C}$, which gives

$$
\begin{aligned}
\xi(z) \overline{\xi(\overline{\eta(z)})} & =c e^{-a \bar{b} z} \bar{c} e^{-\bar{a} b \eta(z)}=|c|^{2} e^{-a \bar{b} z-\bar{a} b(a z+b)} \\
& =|c|^{2} e^{-\bar{a} b^{2}} e^{-\left(a \bar{b}+b|a|^{2}\right) z} \\
& =|c|^{2} e^{-\bar{a} b^{2}} e^{-(a \bar{b}+b) z}, \quad(\text { because }|a|=1) \\
& =|c|^{2} e^{-\bar{a} b^{2}}, \quad(\text { because } a \bar{b}+b=0) \\
& =|c|^{2} e^{|b|^{2}}, \quad(\text { because } \bar{a} b=-\bar{b})
\end{aligned}
$$

Then equation 2.13 gives $|c|^{2} e^{|b|^{2}}=1$.

- Conversely, suppose that the symbols $\xi, \eta$ are of forms (2.7), with conditions (2.10). Then we have

$$
\begin{aligned}
\xi(z) \overline{\xi(\overline{\eta(z)})} & =|c|^{2} e^{-\bar{a} b^{2}} e^{-\left(a \bar{b}+b|a|^{2}\right) z}=|c|^{2} e^{-\bar{a} b^{2}} e^{-(a \bar{b}+b) z} \\
& \left.=|c|^{2} e^{-\bar{a} b^{2}}, \quad \text { (because } a \bar{b}+b=0\right), \\
& =|c|^{2} e^{|b|^{2}}, \quad(\text { because } \bar{a} b=-\bar{b}), \\
& =1,
\end{aligned}
$$


and

$$
\begin{aligned}
\overline{\eta(\overline{\eta(z)})} & =|a|^{2} z+\bar{a} b+\bar{b} \quad(\text { by }(2.11)) \\
& =z \quad(\text { by } 2.10) .
\end{aligned}
$$

From these, we have 2.12, which implies that $\mathcal{A}_{\xi, \eta}$ is involutive.

Remark 2.1.17. By Propositions 2.1.14 2.1.16, an involutive anti-linear weighted composition operator $\mathcal{A}_{\xi, \eta}$ must necessarily be isometric, but the inverse direction fails to hold. Indeed, $(a, b, c)=\left(1,1+i, e^{-1}\right)$ satisfy (2.8), but 2.10) is not satisfied $(a \bar{b}+b \neq 0)$.

\subsubsection{Conjugation}

Combining Propositions 2.1.14-2.1.16 yields the following important criterion.

Theorem 2.1.18. Let $\mathcal{A}_{\xi, \eta}$ be a bounded anti-linear weighted composition operator, induced by two entire functions $\xi, \eta$ with $\xi \not \equiv \mathbf{0}$. The following assertions hold:

1. The anti-linear weighted composition operator $\mathcal{A}_{\xi, \eta}$ is a conjugation on $\mathcal{F}^{2}(\mathbb{C})$.

2. The anti-linear weighted composition operator $\mathcal{A}_{\xi, \eta}$ is involutive on $\mathcal{F}^{2}(\mathbb{C})$.

3. The symbols are of forms

$$
\eta(z)=a z+b, \quad \xi(z)=c e^{-a \bar{b} z}
$$

where $a, b$, and $c$ are complex constants satisfying conditions 2.10, i.e.

$$
|a|=1, \quad \bar{a} b+\bar{b}=0, \quad|c|^{2} e^{|b|^{2}}=1
$$

Proof. Clearly, (1) $\Longrightarrow(2)$ by definition of a conjugation.

The equivalence $(2) \Longleftrightarrow(3)$ holds by Proposition 2.1.16, while the implication $(2) \Longrightarrow(1)$ holds by Remark 2.1 .17 . 
We call operators, obtained in Theorem 2.1.18, as the weighted composition conjugations and denote by $\mathcal{C}_{a, b, c}$, that is

$$
\mathcal{C}_{a, b, c} f(z):=c e^{b z} \overline{f(\overline{a z+b})}, \quad \text { where }|a|=1, \bar{a} b+\bar{b}=0,|c|^{2} e^{|b|^{2}}=1
$$

Clearly, $\mathcal{C}_{1,0,1}=\mathcal{J}$ is the standard conjugation studied on Hardy spaces.

\subsection{Linear weighted composition operators}

In this section, we consider a bounded linear weighted composition operator $W_{\psi, \varphi}$ on $\mathcal{F}^{2}(\mathbb{C})$, and study spectral properties as well as a complex symmetry of this operator. Note that by Proposition 1.4.1 a boundedness of the operator $W_{\psi, \varphi}$ always gives $\varphi(z)=A z+B$, where $A, B$ are complex constants with $|A| \leq 1$. In this section, notations $A, B$ are used to indicate these values.

\subsubsection{Some spectral properties}

In this subsection, we give some spectral properties of $W_{\psi, \varphi}$ and $W_{\psi, \varphi}^{*}$ on the Fock space $\mathcal{F}^{2}(\mathbb{C})$. Note that the arguments of the results here, in general, are similar to those of classical Hardy spaces (see [7, Proposition 2.6]).

Identity 1.8 tells us the action of the adjoint operator $W_{\psi, \varphi}^{*}$ on kernel functions. Now we investigate this action on the $m$-th derivative evaluation kernel defined by $K_{z}^{[m]}(u)=u^{m} K_{z}(u)$. Note that these kernels satisfy the following equality

$$
f^{(m)}(u)=\left\langle f, K_{u}^{[m]}\right\rangle, \quad \forall m \in \mathbb{N}, \forall f \in \mathcal{F}^{2}(\mathbb{C}), \forall u \in \mathbb{C}
$$

Proposition 2.2.1. Let $W_{\psi, \varphi}$ be a bounded linear weighted composition operator on $\mathcal{F}^{2}(\mathbb{C})$, induced by two entire functions $\psi, \varphi$ with $\psi \not \equiv \mathbf{0}$. Then for each $m \geq 1$,

$$
W_{\psi, \varphi}^{*} K_{z}^{[m]}=\overline{\psi(z) A^{m}} K_{\varphi(z)}^{[m]}+\sum_{j=0}^{m-1} \overline{h_{j, m}(z)} K_{\varphi(z)}^{[j]}, \quad m \geq 1,
$$


where $h_{j, m}(m \geq 1,0 \leq j \leq m-1)$ are defined recursively as follows

$$
h_{j, m}(z)=\left\{\begin{array}{l}
h_{0, m-1}^{\prime}(z), \quad j=0, \\
A^{m} \psi(z), \quad j=m, \\
A h_{j-1, m-1}(z)+h_{j, m-1}^{\prime}(z), \quad 1 \leq j \leq m-1,
\end{array} \quad h_{0,1}(z)=\psi^{\prime}(z) .\right.
$$

Proof. We prove (2.16) by induction along $m$. Take arbitrarily $f \in \mathcal{F}^{2}(\mathbb{C})$.

For $m=1$, we have

$$
\begin{aligned}
\left\langle W_{\psi, \varphi} f, K_{z}^{[1]}\right\rangle & =\left(W_{\psi, \varphi} f\right)^{\prime}(z)=(\psi \cdot f \circ \varphi)^{\prime}(z) \\
& =\psi(z) \varphi^{\prime}(z) f^{\prime}(\varphi(z))+\psi^{\prime}(z) f(\varphi(z)) \\
& =\left\langle f, \overline{\psi(z) \varphi^{\prime}(z)} K_{\varphi(z)}^{[1]}+\overline{\psi^{\prime}(z)} K_{\varphi(z)}\right\rangle,
\end{aligned}
$$

which implies, due to the arbitrariness of $f$, that

$$
\begin{aligned}
W_{\psi, \varphi}^{*} K_{z}^{[1]} & =\overline{\psi(z) \varphi^{\prime}(z)} K_{\varphi(z)}^{[1]}+\overline{\psi^{\prime}(z)} K_{\varphi(z)} \\
& \left.=\overline{A \psi(z)} K_{\varphi(z)}^{[1]}+\overline{\psi^{\prime}(z)} K_{\varphi(z)} \quad \text { (because } \varphi(z)=A z+B\right)
\end{aligned}
$$

Thus, 2.16 holds for $m=1$. Suppose that 2.16 holds for $m=k$, i.e.

$$
W_{\psi, \varphi}^{*} K_{z}^{[k]}=\sum_{j=0}^{k} \overline{h_{j, k}(z)} K_{\varphi(z)}^{[j]}
$$

We prove that 2.16 is valid for $m=k+1$. Indeed, note that

$$
\begin{aligned}
\left(W_{\psi, \varphi} f\right)^{(k)}(z) & =\left\langle W_{\psi, \varphi} f, K_{z}^{[k]}\right\rangle=\left\langle f, W_{\psi, \varphi}^{*} K_{z}^{[k]}\right\rangle \\
& =\left\langle f, \sum_{j=0}^{k} \overline{h_{j, k}(z)} K_{\varphi(z)}^{[j]}\right\rangle=\sum_{j=0}^{k} h_{j, k}(z)\left\langle f, K_{\varphi(z)}^{[j]}\right\rangle \\
& =\sum_{j=0}^{k} h_{j, k}(z) f^{(j)}(\varphi(z)),
\end{aligned}
$$


which implies, as $\left(W_{\psi, \varphi} f\right)^{(k+1)}=\left(\left(W_{\psi, \varphi} f\right)^{(k)}\right)^{\prime}$, that

$$
\begin{aligned}
\left(W_{\psi, \varphi} f\right)^{(k+1)}(z)= & \sum_{j=0}^{k} h_{j, k}^{\prime}(z) f^{(j)}(\varphi(z))+\sum_{j=0}^{k} h_{j, k}(z) \varphi^{\prime}(z) f^{(j+1)}(\varphi(z)) \\
= & h_{k, k}(z) \varphi^{\prime}(z) f^{(k+1)}(\varphi(z))+h_{0, k}^{\prime}(z) f(\varphi(z)) \\
& +\sum_{j=1}^{k}\left(h_{j-1, k}(z) \varphi^{\prime}(z)+h_{j, k}^{\prime}(z)\right) f^{(j)}(\varphi(z)) .
\end{aligned}
$$

Setting

$$
h_{j, k+1}(z)=\left\{\begin{array}{l}
h_{0, k}^{\prime}(z), \quad j=0, \\
h_{k, k}(z) \varphi^{\prime}(z)=A^{k+1} \psi(z), \quad j=k+1, \\
h_{j-1, k}(z) \varphi^{\prime}(z)+h_{j, k}^{\prime}(z), \quad 1 \leq j \leq k,
\end{array}\right.
$$

we can rewrite

$$
\begin{aligned}
\left(W_{\psi, \varphi} f\right)^{(k+1)}(z) & =h_{k, k}(z) \varphi^{\prime}(z) f^{(k+1)}(\varphi(z))+\sum_{j=0}^{k} h_{j, k+1}(z) f^{(j)}(\varphi(z)) \\
& =\psi(z) A^{k+1}\left\langle f, K_{\varphi(z)}^{[k+1]}\right\rangle+\sum_{j=0}^{k} h_{j, k+1}(z)\left\langle f, K_{\varphi(z)}^{[j]}\right\rangle \\
& =\left\langle f, \overline{\psi(z) A^{k+1}} K_{\varphi(z)}^{[k+1]}+\sum_{j=0}^{k} \overline{h_{j, k+1}(z)} K_{\varphi(z)}^{[j]}\right\rangle .
\end{aligned}
$$

Note that the second equality holds because $\varphi(z)=A z+B$.

The last equality gives

$$
\left\langle W_{\psi, \varphi} f, K_{z}^{[k+1]}\right\rangle=\left(W_{\psi, \varphi} f\right)^{(k+1)}(z)=\left\langle f, \overline{\psi(z) A^{k+1}} K_{\varphi(z)}^{[k+1]}+\sum_{j=0}^{k} \overline{h_{j, k+1}(z)} K_{\varphi(z)}^{[j]}\right\rangle,
$$

which implies, due to the arbitrariness of $f$, that

$$
W_{\psi, \varphi}^{*} K_{z}^{[k+1]}=\overline{\psi(z) A^{k+1}} K_{\varphi(z)}^{[k+1]}+\sum_{j=0}^{k} \overline{h_{j, k+1}(z)} K_{\varphi(z)}^{[j]} .
$$

The proof is complete. 
Identity (1.8) also tells us another interesting fact: if $\varphi$ has a fixed point $d$ (that is $A \neq 1$, and $\left.d=\frac{B}{1-A}\right)$, then $\overline{\psi(d)}$ is an eigenvalue of $W_{\psi, \varphi}^{*}$ corresponding to the eigenvector $K_{d}$. With the help of expression (2.16), we prove a more general result: $\overline{\psi(d) A^{k}}(k \in \mathbb{N})$ are eigenvalues of $W_{\psi, \varphi}^{*}$.

Proposition 2.2.2. Let $W_{\psi, \varphi}$ be a bounded linear weighted composition operator on $\mathcal{F}^{2}(\mathbb{C})$, induced by two entire functions $\psi, \varphi$ with $\psi \not \equiv \mathbf{0}$ and $\varphi \not \equiv$ const (that is $A \neq 0)$. Suppose that $\varphi$ has a fixed point $d$ with $\psi(d) \neq 0$.

1. If $A$ is not a root of unity, then each element $K_{d}^{[m]}$ is a linear combination of elements $v_{0}, \ldots, v_{m}$, where $v_{j}$ are eigenvectors of $W_{\psi, \varphi}^{*}$ corresponding to eigenvalues $\overline{\psi(d) A^{j}}$ whenever $m \in \mathbb{N}$.

2. If $A$ is an $n$-th root of unity (here $n \geq 1$ a positive integer), then each element $K_{d}^{[m]}$ is a linear combination of elements $v_{0}, \ldots, v_{m}$, where $v_{j}$ are eigenvectors of $W_{\psi, \varphi}^{*}$ corresponding to eigenvalues $\overline{\psi(d) A^{j}}$ whenever $0 \leq m \leq n-1$.

Proof. 1) Suppose that $A$ is not a root of unity. We prove (1) by induction. As discussed previously, by (1.8), assertion (1) is true when $m=0$. Suppose that assertion (1) is true whenever $m \leq k$. This means that for every $m \leq k, K_{d}^{[m]}$ is a linear combination of eigenvectors $v_{0}, \ldots, v_{m}$. Note that $\varphi(d)=d$, and by (2.16), $W_{\psi, \varphi}^{*} K_{d}^{[k+1]}$ equals $\overline{\psi(d) A^{k+1}} K_{d}^{[k+1]}$ plus a linear combination of eigenvectors $v_{0}, \ldots, v_{k}$. Thus, we can write

$$
W_{\psi, \varphi}^{*} K_{d}^{[k+1]}=\overline{\psi(d) A^{k+1}} K_{d}^{[k+1]}+\sum_{j=0}^{k} \beta_{j} v_{j}
$$

where $\beta_{j}=\beta_{j}(d, k), j \in\{0, \ldots, k\}$ are complex constants. Putting

$$
v_{k+1}=K_{d}^{[k+1]}+\sum_{j=0}^{k} \frac{\beta_{j}}{\overline{\psi(d)}\left(\overline{A^{k+1}}-\overline{A^{j}}\right)} v_{j} \quad(\text { as } \psi(d) \neq 0, A \neq 0),
$$


we have

$$
\begin{aligned}
W_{\psi, \varphi}^{*} v_{k+1} & =W_{\psi, \varphi}^{*} K_{d}^{[k+1]}+\sum_{j=0}^{k} \frac{\beta_{j}}{\overline{\psi(d)}\left(\overline{A^{k+1}}-\overline{A^{j}}\right)} W_{\psi, \varphi}^{*} v_{j} \\
& =W_{\psi, \varphi}^{*} K_{d}^{[k+1]}+\sum_{j=0}^{k} \overline{\overline{\psi(d)}\left(\overline{A^{k+1}}-\overline{A^{j}}\right)} \overline{\psi(d) A^{j}} v_{j} \quad\left(v_{j} \text { is an eigenvector }\right) .
\end{aligned}
$$

By 2.17), we see

$$
\begin{aligned}
W_{\psi, \varphi}^{*} v_{k+1} & =\overline{\psi(d) A^{k+1}} K_{d}^{[k+1]}+\sum_{j=0}^{k} \beta_{j} v_{j}+\sum_{j=0}^{k} \frac{\beta_{j} \overline{A^{j}}}{\overline{A^{k+1}}-\overline{A^{j}}} v_{j} \\
& =\overline{\psi(d) A^{k+1}} K_{d}^{[k+1]}+\sum_{j=0}^{k} \frac{\beta_{j} \overline{A^{k+1}}}{\overline{A^{k+1}}-\overline{A^{j}}} v_{j} \\
& =\overline{\psi(d) A^{k+1}} v_{k+1} .
\end{aligned}
$$

2) The case when $A$ is an $n$-th root of unity is proved similarly.

Having discussed the point spectrum of $W_{\psi, \varphi}^{*}$, we pass to studying the point spectrum of $W_{\psi, \varphi}$ on the Fock space $\mathcal{F}^{2}(\mathbb{C})$.

Proposition 2.2.3. Let $W_{\psi, \varphi}$ be a bounded linear weighted composition operator on $\mathcal{F}^{2}(\mathbb{C})$, induced by two entire functions $\psi, \varphi$ with $\psi \not \equiv \mathbf{0}$. If $\varphi$ has a fixed point $d$ (that is $\varphi(d)=d$ ), then we have $\sigma_{p}\left(W_{\psi, \varphi}\right) \subseteq\left\{A^{k} \psi(d): k \in \mathbb{N}\right\}$.

In particular, with the case when $\varphi$ is a constant function (that is $A=0$, and so $d=B)$, the set inclusion admits the equality. Namely, $\sigma_{p}\left(W_{\psi, \varphi}\right)=\{\psi(B), 0\}$.

Proof. Take $\mu \in \sigma_{p}\left(W_{\psi, \varphi}\right)$. Then there exists $f \in \mathcal{F}^{2}(\mathbb{C}) \backslash\{\mathbf{0}\}$ such that

$$
W_{\psi, \varphi} f=\mu f .
$$

- If $f(d) \neq 0$, then

$$
\psi(d) f(d)=\psi(d) f(\varphi(d))=W_{\psi, \varphi} f(d)=\mu f(d)
$$


which gives $\mu=\psi(d)$.

- If $f$ has $d$ as a zero with multiplicity $q \geq 1$, then

$$
f^{(q)}(d) \neq 0, \quad f^{(j)}(d)=0, \forall j \in\{0,1, \cdots q-1\} .
$$

By 2.16), we have

$$
W_{\psi, \varphi}^{*} K_{d}^{[q]}=\overline{\psi(d) A^{q}} K_{d}^{[q]}+\sum_{j=0}^{q-1} \overline{h_{j, q}(d)} K_{d}^{[j]}
$$

which gives

$$
\begin{aligned}
\left\langle W_{\psi, \varphi} f, K_{d}^{[q]}\right\rangle & =\left\langle f, W_{\psi, \varphi}^{*} K_{d}^{[q]}\right\rangle=\left\langle f, \overline{\psi(d) A^{q}} K_{d}^{[q]}+\sum_{j=0}^{q-1} \overline{h_{j, q}(d)} K_{d}^{[j]}\right\rangle \\
& =\psi(d) A^{q} f^{(q)}(d)+\sum_{j=0}^{q-1} h_{j, q}(d) f^{(j)}(d)=\psi(d) A^{q} f^{(q)}(d)
\end{aligned}
$$

On the other hand, since $W_{\psi, \varphi} f=\mu f$, we have

$$
\left\langle W_{\psi, \varphi} f, K_{d}^{[q]}\right\rangle=\left\langle\mu f, K_{d}^{[q]}\right\rangle=\mu f^{(q)}(d) .
$$

Consequently, $\mu=\psi(d) A^{q}$.

For the second conclusion, suppose that $A=0$, and then $d=B$. By the first conclusion, $\sigma_{p}\left(W_{\psi, \varphi}\right) \subseteq\{\psi(B), 0\}$. To prove the reverse inclusion, we attempt to find eigenvectors.

Note that $\psi \in \mathcal{F}^{2}(\mathbb{C}) \backslash\{\mathbf{0}\}$, and

$$
W_{\psi, \varphi} \psi=\psi \cdot \psi \circ \varphi=\psi(B) \psi
$$

which gives $\psi(B) \in \sigma_{p}\left(W_{\psi, \varphi}\right)$.

On the other hand, the function $f(z)=z-B \in \mathcal{F}^{2}(\mathbb{C}) \backslash\{\mathbf{0}\}$ satisfies

$$
W_{\psi, \varphi} f=\psi \cdot f \circ \varphi=\psi \cdot f(B)=\mathbf{0},
$$

and hence, $0 \in \sigma_{p}\left(W_{\psi, \varphi}\right)$. 


\subsubsection{Complex symmetry with an arbitrary conjugation}

In this subsection, we investigate a complex symmetry in the Fock space $\mathcal{F}^{2}(\mathbb{C})$. We start with proving some properties of $\psi$ and $\varphi$ when $W_{\psi, \varphi}$ is complex symmetric on the Fock space $\mathcal{F}^{2}(\mathbb{C})$ with a arbitrary conjugation. Note that the arguments of the below result are, in general, similar to those of classical Hardy spaces (see [7], [15]).

Proposition 2.2.4. Let $\mathcal{C}$ a conjugation on $\mathcal{F}^{2}(\mathbb{C})$, and $W_{\psi, \varphi}$ a $\mathcal{C}$-symmetric bounded weighted composition operator on $\mathcal{F}^{2}(\mathbb{C})$ induced by two entire functions $\varphi \not \equiv$ const,$\psi \not \equiv \mathbf{0}$. Then the following statements hold:

1. The function $\psi$ is of the form $\psi(z)=\psi(0) e^{\alpha z^{2}+\beta z}$, where $\alpha, \beta$ are complex constants satisfying the conditions $|\alpha|<\frac{1}{2}$ and $\psi(0) \neq 0$.

2. There is the identity

$$
\psi(u) \mathcal{C} K_{\varphi(u)}(z)=\psi(z) \cdot\left(\mathcal{C} K_{u}\right) \circ \varphi(z), \quad \forall z, u \in \mathbb{C}
$$

3. $\operatorname{ker}\left(W_{\psi, \varphi}\right)=\{\mathbf{0}\}$, while $\operatorname{Im}\left(W_{\psi, \varphi}\right)$ is dense in $\mathcal{F}^{2}(\mathbb{C})$.

Proof. 1) By [13, Theorem 1.1], it is enough to show that $\psi$ is nowhere vanished on $\mathbb{C}$. Assume in contrary that $\psi(\alpha)=0$ for some $\alpha \in \mathbb{C}$. Then by $[23$, Conclusion 1.2.12], there is a neighborhood $V$ of $\alpha$ such that $\psi(z) \neq 0$ for every $z \in V \backslash\{\alpha\}$. By (1.8),

$$
W_{\psi, \varphi}^{*} K_{\alpha}=\overline{\psi(\alpha)} K_{\varphi(\alpha)}=\mathbf{0} .
$$

But $W_{\psi, \varphi}$ is $\mathcal{C}$-symmetric, hence

$$
W_{\psi, \varphi} \mathcal{C} K_{\alpha}=\mathcal{C} W_{\psi, \varphi}^{*} K_{\alpha}=\mathcal{C} \mathbf{0}=\mathbf{0}
$$

The last equation implies that for every $z \in \mathbb{C}$

$$
\psi(z) \mathcal{C} K_{\alpha}(\varphi(z))=W_{\psi, \varphi} \mathcal{C} K_{\alpha}(z)=0
$$


which shows that $\mathcal{C} K_{\alpha} \circ \varphi=\mathbf{0}$ on $V \backslash\{\alpha\}$. Consequently, $\mathcal{C} K_{\alpha}=\mathbf{0}$, which implies, by the involutivity of $\mathcal{C}$, that $K_{\alpha}=\mathbf{0}$ : a contradiction.

2) For $z, u \in \mathbb{C}$, we have

$$
\begin{aligned}
\psi(u) \mathcal{C} K_{\varphi(u)}(z) & =\mathcal{C}\left(\overline{\psi(u)} K_{\varphi(u)}\right)(z) \quad \text { (because } \mathcal{C} \text { is anti-linear) } \\
& =\mathcal{C}\left(W_{\psi, \varphi}^{*} K_{u}\right)(z) \quad(\text { by }(1.8)) \\
& =W_{\psi, \varphi} \mathcal{C} K_{u}(z) \quad \text { (because } W_{\psi, \varphi} \text { is } \mathcal{C} \text {-symmetric) } \\
& =\psi(z) \cdot\left(\mathcal{C} K_{u}\right) \circ \varphi(z) .
\end{aligned}
$$

3) Let $g \in \operatorname{ker}\left(W_{\psi, \varphi}\right)$. For every $z \in \mathbb{C}$, we have

$$
\psi(z) g(\varphi(z))=W_{\psi, \varphi} g(z)=0,
$$

which implies, as $\psi$ is nowhere vanished, that $g(\varphi(z))=0$. This implies, as $\varphi$ is non-constant, that $g \equiv \mathbf{0}$. Thus, $\operatorname{ker}\left(W_{\psi, \varphi}\right)=\{\mathbf{0}\}$.

To prove $\operatorname{Im}\left(W_{\psi, \varphi}\right)$ is dense in $\mathcal{F}^{2}(\mathbb{C})$, since $\mathcal{F}^{2}(\mathbb{C})=\overline{\operatorname{Im}\left(W_{\psi, \varphi}\right)} \oplus \operatorname{ker}\left(W_{\psi, \varphi}^{*}\right)$, it suffices to show that $\operatorname{ker}\left(W_{\psi, \varphi}^{*}\right)=\{\mathbf{0}\}$. Indeed, if $f \in \operatorname{ker}\left(W_{\psi, \varphi}^{*}\right)$, then

$$
\psi \cdot(\mathcal{C} f) \circ \varphi=W_{\psi, \varphi} \mathcal{C} f=\mathcal{C} W_{\psi, \varphi}^{*} f=\mathcal{C} \mathbf{0}=\mathbf{0},
$$

which implies, as $\psi$ is nowhere vanished, that $(\mathcal{C} f) \circ \varphi=0$. This leads, since $\varphi$ is non-constant and $\mathcal{C}$ is involutive, to $f \equiv \mathbf{0}$. Thus, $\operatorname{ker}\left(W_{\psi, \varphi}^{*}\right)=\{\mathbf{0}\}$.

Note that $\lambda$ is the eigenvalue of a complex symmetric bounded operator $T$ if and only if $\bar{\lambda}$ is the eigenvalue of $T^{*}$. This fact, together with Proposition 2.2.2, shows that $A^{k} \psi(d), k \in \mathbb{N}$ are eigenvalues of $W_{\psi, \varphi}$. By Proposition 2.2.3, we observe that eigenvalues of $W_{\psi, \varphi}$ are only these values.

Theorem 2.2.5. Let $\mathcal{C}$ a conjugation on $\mathcal{F}^{2}(\mathbb{C})$, and $W_{\psi, \varphi}$ a $\mathcal{C}$-symmetric bounded weighted composition operator on $\mathcal{F}^{2}(\mathbb{C})$ induced by two entire functions $\varphi, \psi$ with $\psi \not \equiv 0$. If $\varphi$ has a fixed point d (that is $A \neq 1$ ), then

$$
\sigma_{p}\left(W_{\psi, \varphi}\right)=\left\{A^{k} \psi(d): k \in \mathbb{N}\right\}
$$


Remark 2.2.6. We note that in Theorem 2.2.5, the eigenvectors corresponding to the eigenvalues $\psi(d) A^{n}$, in general, can not be indicated explicitly. However, as it is seen below in next section, in the case of weighted composition conjugations, we can provide an explicit formula.

\subsubsection{Complex symmetry with weighted composition con- jugations}

In this subsection, we consider a bounded linear weighted composition operator $W_{\psi, \varphi}$ and study its $\mathcal{C}_{a, b, c}$-symmetry.

To investigate the necessity for the $\mathcal{C}_{a, b, c^{-}}$-symmetry, we apply the symmetric condition $\mathcal{C}_{a, b, c} W_{\psi, \varphi}^{*}=W_{\psi, \varphi} \mathcal{C}_{a, b, c}$ to kernel functions. It turns out that the action on only $K_{0}$ can already give the forms for the symbols, which are also the sufficient condition.

Proposition 2.2.7. Let $\mathcal{C}_{a, b, c}$ be a weighted composition conjugation on $\mathcal{F}^{2}(\mathbb{C})$ defined by 2.15), and $W_{\psi, \varphi}$ a bounded linear weighted composition operator on $\mathcal{F}^{2}(\mathbb{C})$ induced by two entire functions $\psi, \varphi$ with $\psi \not \equiv \mathbf{0}$. If the operator $W_{\psi, \varphi}$ satisfies

$$
W_{\psi, \varphi} \mathcal{C}_{a, b, c} K_{0}=\mathcal{C}_{a, b, c} W_{\psi, \varphi}^{*} K_{0}
$$

then

$$
\varphi(z)=A z+B, \quad \psi(z)=C e^{D z}, \quad \text { with } C \neq 0, D=a B-b A+b .
$$

Proof. Since $W_{\psi, \varphi}$ is bounded on $\mathcal{F}^{2}(\mathbb{C})$, by Proposition 1.4.1, we have $\varphi(z)=$ $A z+B$ with $|A| \leq 1$.

Since $K_{0}(z) \equiv 1$, we have $\mathcal{C}_{a, b, c} K_{0}(z)=c e^{b z}$ and hence,

$$
W_{\psi, \varphi} \mathcal{C}_{a, b, c} K_{0}(z)=\psi(z) c e^{b \varphi(z)}=\psi(z) c e^{b(A z+B)}, z \in \mathbb{C}
$$


On the other hand, by (1.8), $W_{\psi, \varphi}^{*} K_{0}=\overline{\psi(0)} K_{\varphi(0)}$, which implies that

$$
\begin{aligned}
\mathcal{C}_{a, b, c} W_{\psi, \varphi}^{*} K_{0}(z) & =\mathcal{C}_{a, b, c}\left(\overline{\psi(0)} K_{\varphi(0)}\right)(z)=\psi(0) \mathcal{C}_{a, b, c}\left(K_{\varphi(0)}\right)(z) \\
& =c e^{b z} \psi(0) e^{\varphi(0)(a z+b)}=c \psi(0) e^{(a B+b) z+b B}
\end{aligned}
$$

Thus, the equality $W_{\psi, \varphi} \mathcal{C}_{a, b, c} K_{0}=\mathcal{C}_{a, b, c} W_{\psi, \varphi}^{*} K_{0}$ is reduced to

$$
\psi(z)=\psi(0) e^{(a B-b A+b) z}=C e^{D z},
$$

where $C=\psi(0) \neq 0($ since $\psi \not \equiv \mathbf{0})$ and $D=a B-b A+b$.

Now with the help of Proposition 2.1.13, we can establish the following criterion.

Theorem 2.2.8. Let $\mathcal{C}_{a, b, c}$ be a weighted composition conjugation defined by 2.15), and $W_{\psi, \varphi}$ a bounded linear weighted composition operator on $\mathcal{F}^{2}(\mathbb{C})$ induced by two entire functions $\psi, \varphi$ with $\psi \neq \equiv \mathbf{0}$. Then $W_{\psi, \varphi}$ is $\mathcal{C}_{a, b, c}$-symmetric if and only if the symbols are of the following forms:

$$
\varphi(z)=A z+B, \psi(z)=C e^{D z}, \text { with } C \neq 0, D=a B-b A+b,
$$

where $A, B$ are complex constants satisfying

$$
\left\{\begin{array}{l}
\text { either }|A|<1 \\
\text { or }|A|=1, D+A \bar{B}=0 .
\end{array}\right.
$$

Proof. • Suppose $W_{\psi, \varphi}$ is $\mathcal{C}_{a, b, c}$-symmetric on $\mathcal{F}^{2}(\mathbb{C})$. By Proposition 2.2.7, the symbols must be of forms 2.18.

Furthermore, since $W_{\psi, \varphi}$ is bounded and $\psi(z)=C e^{D z}$, by Proposition 2.1.13. we get 2.19.

- Conversely, suppose $\psi$ and $\varphi$ satisfy conditions (2.18)-2.19). For every $z, u \in$ $\mathbb{C}$, by 1.8 , we have

$$
\begin{aligned}
\left(\mathcal{C}_{a, b, c} W_{\psi, \varphi}^{*} K_{z}\right)(u) & =c e^{b u} \overline{\left(W_{\psi, \varphi}^{*} K_{z}\right)(\overline{a u+b})}=c e^{b u} \overline{\overline{\psi(z)} K_{\varphi(z)}(\overline{a u+b})} \\
& =c e^{b u} \psi(z) e^{\varphi(z)(a u+b)}=c e^{b u} C e^{D z} e^{(A z+B)(a u+b)} \\
& =c C e^{a A u z+(A b+D) z+(a B+b) u+b B}
\end{aligned}
$$


On the other hand,

$$
\begin{aligned}
\left(W_{\psi, \varphi} \mathcal{C}_{a, b, c} K_{z}\right)(u) & =\psi(u)\left(\mathcal{C}_{a, b, c} K_{z}\right)(\varphi(u))=\psi(u) c e^{b \varphi(u)} \overline{K_{z}(\overline{a \varphi(u)+b})} \\
& =\psi(u) c e^{b \varphi(u)} e^{z(a \varphi(u)+b)}=C e^{D u} c e^{b(A u+B)} e^{z(a(A u+B)+b)} \\
& =c C e^{a A u z+(a B+b) z+(A b+D) u+b B}
\end{aligned}
$$

Since $D=a B-b A+b$, by 2.18 , we can write

$$
\begin{aligned}
\left(W_{\psi, \varphi} \mathcal{C}_{a, b, c} K_{z}\right)(u) & =c C e^{a A u z+(A b+D) z+(a B+b) u+b B} \\
& =\left(\mathcal{C}_{a, b, c} W_{\psi, \varphi}^{*} K_{z}\right)(u)
\end{aligned}
$$

which gives

$$
W_{\psi, \varphi} \mathcal{C}_{a, b, c} K_{z}=\mathcal{C}_{a, b, c} W_{\psi, \varphi}^{*} K_{z}, \quad \forall z \in \mathbb{C}
$$

Since the span of the kernel functions is dense in $\mathcal{F}^{2}(\mathbb{C})$, and $W_{\psi, \varphi}$ is bounded, by Lemma 1.1.7,

$$
W_{\psi, \varphi} \mathcal{C}_{a, b, c} f=\mathcal{C}_{a, b, c} W_{\psi, \varphi}^{*} f, \quad \forall f \in \mathcal{F}^{2}(\mathbb{C})
$$

which means that $W_{\psi, \varphi}$ is $\mathcal{C}_{a, b, c}$-symmetric.

The lemma below is useful in studying eigenvectors of a $\mathcal{C}_{a, b, c}$-symmetric bounded weighted composition operator $W_{\psi, \varphi}$ on the Fock space $\mathcal{F}^{2}(\mathbb{C})$.

Lemma 2.2.9. For every $n \in \mathbb{N}, s \in \mathbb{R}$, we always have

$$
\int_{\mathbb{C}}|z|^{n} e^{2 s|z|-|z|^{2}} d V(z)<\infty .
$$

Proof. For each $n \in \mathbb{N}$, the integral

$$
I_{n}(R)=\int_{|z|<R}|z|^{n} e^{2 s|z|-|z|^{2}} d V(z), \quad R>s+1,
$$


by changing the variables $z=r e^{i \theta}$, is written as

$$
\begin{aligned}
I_{n}(R) & =\int_{0}^{2 \pi} d \theta \int_{0}^{R} r^{n+1} e^{2 s r-r^{2}} d r=2 \pi \int_{0}^{R} r^{n+1} e^{2 s r-r^{2}} d r \\
& =2 \pi\left(\int_{0}^{s+1}+\int_{s+1}^{R}\right) r^{n+1} e^{2 s r-r^{2}} d r \\
& =2 \pi \int_{0}^{s+1} r^{n+1} e^{2 s r-r^{2}} d r+2 \pi e^{s^{2}} \int_{s+1}^{R} r^{n+1} e^{-(r-s)^{2}} d r .
\end{aligned}
$$

Since $e^{t} \geq \frac{t^{n+2}}{(n+2) !}$, we estimate

$$
\begin{aligned}
I_{n}(R) & \leq 2 \pi \int_{0}^{s+1} r^{n+1} e^{2 s r-r^{2}} d r+2 \pi e^{s^{2}}(n+2) ! \int_{s+1}^{R} \frac{r^{n+1}}{(r-s)^{2 n+4}} d r \\
& =2 \pi \int_{0}^{s+1} r^{n+1} e^{2 s r-r^{2}} d r+2 \pi e^{s^{2}}(n+2) ! \int_{1}^{R-s} \frac{(r+s)^{n+1}}{r^{2 n+4}} d r .
\end{aligned}
$$

From this, it follows that $I_{n}=\lim _{R \rightarrow \infty} I_{n}(R)<\infty$.

Our next task is to give the explicit formula for eigenvectors of a $\mathcal{C}_{a, b, c}$-symmetric bounded weighted composition operator $W_{\psi, \varphi}$ in the case when $\varphi$ has one fixed point (that is $A \neq 1$, and the fixed point is $d=\frac{B}{1-A}$ ).

Proposition 2.2.10. Let $\mathcal{C}_{a, b, c}$ be a weighted composition conjugation defined by 2.15, and $W_{\psi, \varphi}$ a $\mathcal{C}_{a, b, c}$-symmetric bounded weighted composition operator on $\mathcal{F}^{2}(\mathbb{C})$ induced by two entire functions $\psi, \varphi$ with $\psi \neq \equiv \mathbf{0}$ (that is Theorem 2.2.8 works). If $\varphi$ has a fixed point $d$ (that is $A \neq 1$, and furthermore, $d=\frac{B}{1-A}$ ), then

$$
g_{k}(z)=(z-d)^{k} e^{D z /(1-A)}, \quad k \in \mathbb{N}
$$

are eigenvectors of $W_{\psi, \varphi}$ corresponding to the eigenvalues $A^{k} \psi(d)$.

Proof. By Proposition 2.2.3 and Theorem 2.2.5, the point spectrum of $W_{\psi, \varphi}$ is

$$
\sigma_{p}\left(W_{\psi, \varphi}\right)=\left\{A^{k} \psi(d): k \in \mathbb{N}\right\}
$$


For each $k \geq 0$, we have

$$
\begin{aligned}
& \left|g_{k}(z)\right|^{2} \leq(|z|+|d|)^{2 k} e^{2 \operatorname{Re}(z \alpha)} \quad\left(\text { where } \alpha=D(1-A)^{-1}\right) \\
& \left.\leq(|z|+|d|)^{2 k} e^{2|z \alpha|} \quad \text { (because } \operatorname{Re}(z \alpha) \leq|z \alpha|\right) \\
& =\sum_{j=0}^{2 k}\left(\begin{array}{c}
2 k \\
j
\end{array}\right)|d|^{j}|z|^{2 k-j} e^{2|z \alpha|} \text {, }
\end{aligned}
$$

which gives

$$
\left\|g_{k}\right\|^{2} \leq \frac{1}{\pi} \sum_{j=0}^{2 k}\left(\begin{array}{c}
2 k \\
j
\end{array}\right)|d|^{j} \int_{\mathbb{C}}|z|^{2 k-j} e^{2|z \alpha|-|z|^{2}} d V(z)<\infty \quad \text { (by Lemma 2.2.9. } .
$$

The last inequality shows $g_{k} \in \mathcal{F}^{2}(\mathbb{C})$. Then by Theorem 2.2.8, we have

$$
\begin{aligned}
W_{\psi, \varphi} g_{k}(z) & =C e^{D z} \cdot(A z+B-d)^{k} e^{\frac{D(A z+B)}{1-A}} \\
& =\psi(d) \cdot(A z+B-d)^{k} e^{\frac{D z}{1-A}} \\
& =\psi(d) \cdot A^{k}(z-d)^{k} e^{\frac{D z}{1-A}}=A^{k} \psi(d) g_{k}(z) .
\end{aligned}
$$

Having discussed the point spectrum, we pass to finding the spectrum of $\mathcal{C}_{a, b, c^{-}}$ symmetric bounded weighted composition operators on the Fock space $\mathcal{F}^{2}(\mathbb{C})$. The following result shows the spectrum of a complex symmetric operator may be complex.

Proposition 2.2.11. Let $\mathcal{C}_{a, b, c}$ be a weighted composition conjugation defined by 2.15 , and $W_{\psi, \varphi}$ a $\mathcal{C}_{a, b, c}$ symmetric bounded weighted composition operator on $\mathcal{F}^{2}(\mathbb{C})$ induced by two entire functions $\psi, \varphi$ with $\psi \not \equiv \mathbf{0}$. Then

$\sigma\left(W_{\psi, \varphi}\right)=\left\{\begin{array}{l}C e^{|B|^{2} / 2} \mathbb{T}, \quad \text { if } A=1 \text { and } D+\bar{B}=0, \\ \psi(d) \cdot \operatorname{Cl}\left(\left\{A^{k}: k \in \mathbb{N}\right\}\right) \text {, if either } A \in \mathbb{T} \backslash\{1\}, D+A \bar{B}=0 \text { or }|A|<1 .\end{array}\right.$

Here, $d=\frac{B}{1-A}$ when $A \neq 1$. 
Proof. - If $|A|<1$ then by (2.4), we have

$$
\lim _{|z| \rightarrow \infty} M_{z}(\psi, \varphi)=0
$$

By Proposition 1.4.1, $W_{\psi, \varphi}$ is compact, and so by the Riesz-Schauder theorem (see [22, Theorem VI.15]),

$$
\sigma\left(W_{\psi, \varphi}\right)=\{0\} \cup \sigma_{p}\left(W_{\psi, \varphi}\right) .
$$

On the other hand, by Proposition 2.2 .3 and Theorem 2.2.5.

$$
\sigma_{p}\left(W_{\psi, \varphi}\right)=\left\{A^{k} \psi(d): k \in \mathbb{N}\right\} .
$$

Thus, the conclusion is true in the case when $|A|<1$ (since $\lim _{k \rightarrow \infty} A^{k}=0$ ).

- Now suppose that $A \in \mathbb{T}$. Since $|A|=1$, from 2.19 it follows that $D=-A \bar{B}$. Then,

$$
W_{\psi, \varphi} f(z)=C e^{-A \bar{B} z} f(A z+B), f \in \mathcal{F}^{2}(\mathbb{C}) .
$$

Set $\phi(z)=e^{-A \bar{B} z-\frac{|B|^{2}}{2}}$. Then $W_{\psi, \varphi}=C e^{\frac{|B|^{2}}{2}} W_{\phi, \varphi}$, and this implies that

$$
\sigma\left(W_{\psi, \varphi}\right)=C e^{\frac{|B|^{2}}{2}} \sigma\left(W_{\phi, \varphi}\right)
$$

By [28, Corollary 1.4], $W_{\phi, \varphi}$ is unitary and

$$
\sigma\left(W_{\phi, \varphi}\right)= \begin{cases}\operatorname{Cl}\left(\left\{A^{k} e^{\frac{|B|^{2}}{2} \cdot \frac{A+1}{A-1}}: k \in \mathbb{N}\right\}\right), & A \neq 1 \\ \mathbb{T}, & A=1\end{cases}
$$

Note that

$$
e^{\frac{|B|^{2}}{2}} e^{\frac{|B|^{2}}{2} \cdot \frac{A+1}{A-1}}=e^{\frac{|B|^{2} A}{A-1}}=e^{\frac{A \bar{B} B}{A-1}}=e^{-A \bar{B} d}=e^{D d} .
$$

If $A=1$, then

$$
\sigma\left(W_{\phi, \varphi}\right)=C e^{|B|^{2} / 2} \mathbb{T}
$$

while if $A \neq 1$, then

$$
\begin{aligned}
\sigma\left(W_{\phi, \varphi}\right) & =C e^{\frac{|B|^{2}}{2}} \cdot \mathrm{Cl}\left(\left\{A^{k} e^{\frac{|B|^{2}}{2} \cdot \frac{A+1}{A-1}}: k \in \mathbb{N}\right\}\right) \\
& =\psi(d) \cdot \mathrm{Cl}\left(\left\{A^{k}: k \in \mathbb{N}\right\}\right) .
\end{aligned}
$$




\subsubsection{Normality}

As mentioned in the Introduction, the class of complex symmetric operators is large enough to cover many well-known operators such as normal operators, unitary operators, etc. So, the question we study in this subsection is: how big is the class of $\mathcal{C}_{a, b, c^{-}}$-symmetric weighted composition operators?.

It turns out that the class of $\mathcal{C}_{a, b, c}$-symmetric weighted composition operators contains properly all normal weighted composition operators.

Theorem 2.2.12. Let $W_{\psi, \varphi}$ be a bounded linear weighted composition operator on $\mathcal{F}^{2}(\mathbb{C})$, induced by entire functions $\varphi, \psi$ with $\psi \not \equiv \mathbf{0}$. If the operator $W_{\psi, \varphi}$ is normal, then it is complex symmetric with respect to some weighted composition conjugation. Moreover, this conjugation can be given explicitly.

Proof. Recall that by Proposition 1.4.3, the operator $W_{\psi, \varphi}$ is normal on the Fock space $\mathcal{F}^{2}(\mathbb{C})$ if and only if the symbols are of the following forms:

1. $\varphi(z)=A z+B$ with $|A|<1$ and $\psi(z)=C e^{D z}$, where $D=\frac{\bar{B}(1-A)}{1-\bar{A}}$.

2. $\varphi(z)=A z+B$ with $|A|=1$ and $\psi(z)=C e^{D z}$, where $D=-A \bar{B}$.

If $A \neq 1$, then we choose

$$
b=0, c=1, a=\frac{\bar{B}(1-A)}{B(1-\bar{A})} .
$$

If $A=1$, then we choose

$$
b=0, c=1, a=\left\{\begin{array}{l}
-\frac{\bar{B}}{B}, \quad B \neq 0, \\
1, \quad B=0 .
\end{array}\right.
$$

With this choice, condition 2.10 holds, and hence, $\mathcal{C}_{a, b, c}$ is a conjugation on $\mathcal{F}^{2}(\mathbb{C})$. By Theorem 2.2.8, the operator $W_{\psi, \varphi}$ is $\mathcal{C}_{a, b, c^{-}}$symmetric on $\mathcal{F}^{2}(\mathbb{C})$. 
As a consequence, Hermitian weighted composition operators, as well as unitary ones, are complex symmetric with respect to some weighted composition conjugation on the Fock space $\mathcal{F}^{2}(\mathbb{C})$.

The below example gives us a $\mathcal{C}_{a, b, c}$-symmetric weighted composition operator, which is not normal.

Example 2.2.13. We consider entire functions $\varphi, \psi$ given by

$$
\varphi(z)=\frac{z}{2}, \quad \psi(z)=e^{\frac{i z}{2}}
$$

In this case,

$$
A=\frac{1}{2}, B=0, C=1, D=\frac{i}{2} .
$$

Clearly, $W_{\psi, \varphi}$ is bounded on $\mathcal{F}^{2}(\mathbb{C})$ by Proposition 2.1.13.

For choosing $a=1, b=i, c=e^{-1 / 2}$, by Theorem 2.1.18, the operator $\mathcal{C}_{a, b, c}$ is a conjugation on $\mathcal{F}^{2}(\mathbb{C})$.

The operator $W_{\psi, \varphi}$ is $\mathcal{C}_{a, b, c}$-symmetric, because

$$
|A|<1, \quad D=\frac{i}{2}=a B-b A+b
$$

and is not normal, because

$$
|A|<1, \quad D=\frac{i}{2} \neq 0=\frac{\bar{B}(1-A)}{1-\bar{A}} .
$$




\section{Chapter 3}

\section{Complex symmetric $C_{0}$-semigroups}

We prove a new version of Stone's theorem. Let $\mathcal{C}$ be a conjugation. If each element of a $C_{0}$-semigroup is also $\mathcal{C}$-symmetric with respect to $\mathcal{C}$, then the generator is $\mathcal{C}$-selfadjoint as an unbounded operator, and vice-versa, if the generator is $\mathcal{C}$ selfadjoint, then this $C_{0}$-semigroup is complex symmetric with respect to $\mathcal{C}$.

These results allow us to introduce a new concept in dynamical systems: complex symmetric $C_{0}$-semigroups $\left(C_{0}\right.$-groups), which contain unitary groups as a particular case. Using the results obtained in Chapter 1, we characterize the structure of a family of weighted composition operators, when it is a $\mathcal{C}_{a, b, c}$-symmetric $C_{0^{-}}$ semigroups on the Fock space $\mathcal{F}^{2}(\mathbb{C})$. In addition, we construct a $\mathcal{C}_{a, b, c}$-symmetric $C_{0}$-semigroup $\left(C_{0}\right.$-groups), which is not unitary. Thus, the concept of complex symmetric $C_{0}$-semigroups $\left(C_{0}\right.$-group) is really a generalization. 


\subsection{Motivation}

In functional analysis, Stone's theorem on $C_{0}$-groups of unitary operators (unitary group for short) is a basic theorem that establishes a one-to-one correspondence between dynamical systems and selfadjoint operators. We recall here this popular result, whose proof can be found in the monographs on operator theory (see e.g. [24]).

Theorem 3.1.1 (Stone's theorem). If $(U(t))_{t \in \mathbb{R}}$ is a unitary group, then there is a unique selfadjoint operator $A$ such that $i A$ generates $(U(t))_{t \in \mathbb{R}}$.

Conversely, we can always define a unitary group from a given selfadjoint operator by functional calculus

$$
e^{i t A}=\int_{\mathbb{R}} e^{i t \lambda} d E_{A}(\lambda), t \in \mathbb{R},
$$

where $A$ is a selfadjoint operator with the spectral measure $E_{A}$.

Motivated by Stone's theorem, we discuss in this chapter the following two questions.

B1. For given a $C_{0}$-semigroup, suppose that the generator is $\mathcal{C}$-selfadjoint. Is this $C_{0}$-semigroup itself complex symmetric?

B2. Conversely, suppose that each element of a $C_{0}$-semigroup is complex symmetric with respect to a fixed conjugation. Is its generator complex symmetric?

On the other hand, it is well known that every unitary operator is complex symmetric. This allows us to choose a conjugation $\mathcal{C}(t)$ such that each element $U(t)$ of a unitary group $\left(U(t)_{t \in \mathbb{R}}\right.$ is $\mathcal{C}(t)$-complex symmetric. Clearly, these conjugations form an operator-valued function from $\mathbb{R}$ into the set of all conjugations on $\mathcal{H}$. So, the next question we are interested in is:

B3. Is there a conjugation, independent of the parameter $t$, that works for all $U(t), t \in \mathbb{R}$ ? 


\subsection{Stone-type theorem}

\subsubsection{Solution to the first question}

In this subsection, we give the answer to B1. The following result is necessary in the proof.

Lemma 3.2.1. If $\mathcal{C}$ is a conjugation on a Hilbert space $\mathcal{H}$, and $f: U \subseteq \mathbb{R} \rightarrow \mathcal{H}$ is a differentiable function, then $(\mathcal{C} f(t))^{\prime}=\mathcal{C} f^{\prime}(t)$, for every $t \in U$.

Proof. Take and fix $t \in U$. Let $\varepsilon>0$ be given. Since $f$ is differentiable on $U$, there exists $\delta=\delta(\varepsilon, t)>0$ such that

$$
\left\|\frac{f(s)-f(t)}{s-t}-f^{\prime}(t)\right\|<\varepsilon
$$

whenever $s \in(t-\delta, t+\delta) \cap U$. Since $\mathcal{C}$ is isometric, we have

$$
\begin{aligned}
& \left\|\frac{\mathcal{C} f(s)-\mathcal{C} f(t)}{s-t}-\mathcal{C} f^{\prime}(t)\right\|=\left\|\mathcal{C}\left(\frac{f(s)-f(t)}{s-t}-f^{\prime}(t)\right)\right\| \\
& =\left\|\frac{f(s)-f(t)}{s-t}-f^{\prime}(t)\right\|<\varepsilon, \quad \forall s \in(t-\delta, t+\delta) \cap U,
\end{aligned}
$$

which gives $(\mathcal{C} f(t))^{\prime}=\mathcal{C} f^{\prime}(t)$.

The following theorem gives the answer for question B1.

Theorem 3.2.2. Let $(T(s))_{s \geq 0}$ be a $C_{0}$-semigroup on a Hilbert space $\mathcal{H}$ with the generator $A$, and $\mathcal{C}: \mathcal{H} \rightarrow \mathcal{H}$ a conjugation. If $A$ is $\mathcal{C}$-selfadjoint, then the operator $T(s)$ is $\mathcal{C}$-symmetric, for every $s \geq 0$.

Proof. Recall that $\left(T(t)^{*}\right)_{t \geq 0}$ is a $C_{0}$-semigroup with the generator $A^{*}$, and hence by [4, Lemma II 1.3],

$$
T(t)^{*} z \in \operatorname{dom}\left(A^{*}\right),\left(T(t)^{*} z\right)^{\prime}=A^{*} T(t)^{*} z=T(t)^{*} A^{*} z, \quad \forall t \geq 0, \forall z \in \operatorname{dom}\left(A^{*}\right) .
$$


Since $A$ is $\mathcal{C}$-selfadjoint, we have

$$
A=\mathcal{C} A^{*} \mathcal{C}, \quad \operatorname{dom}\left(A^{*}\right)=\mathcal{C} \operatorname{dom}(A) .
$$

Let $x$ be an arbitrary element belonging to $\operatorname{dom}(A)$. Then $\mathcal{C} x \in \operatorname{dom}\left(A^{*}\right)$, and hence,

$$
\mathcal{C} T(t)^{*} \mathcal{C} x \in \mathcal{C} T(t)^{*} \operatorname{dom}\left(A^{*}\right) \subseteq \mathcal{C} \operatorname{dom}\left(A^{*}\right)=\mathcal{C} \cdot \mathcal{C} \operatorname{dom}(A)=\operatorname{dom}(A), \quad \forall t \geq 0
$$

We define the function $v: \mathbb{R}_{+} \rightarrow \mathcal{H}$ by

$$
v(t)=\mathcal{C} T(t)^{*} \mathcal{C} x
$$

Clearly, $v(t) \in \operatorname{dom}(A)$, for every $t \geq 0$, and

$$
\begin{aligned}
v^{\prime}(t) & =\left(\mathcal{C} T(t)^{*} \mathcal{C} x\right)^{\prime}=\mathcal{C}\left(T(t)^{*} \mathcal{C} x\right)^{\prime} \quad \text { (by Lemma 3.2.1) } \\
& =\mathcal{C} A^{*} T(t)^{*} \mathcal{C} x \quad(\text { by [4, Lemma II 1.3]) } \\
& \left.=\left(\mathcal{C} A^{*} \mathcal{C}\right)\left(\mathcal{C} T(t)^{*} \mathcal{C}\right) x \quad \text { (by the involutivity of } \mathcal{C}\right) \\
& =A v(t) .
\end{aligned}
$$

These show that $v$ is a classical solution of abstract Cauchy problem (1.6). On the other hand, as mentioned in Section 1.3, abstract Cauchy problem (1.6) has a unique classical solution $u: \mathbb{R}_{+} \rightarrow \mathcal{H}$ given by

$$
u: t \mapsto u(t):=T(t) x
$$

By the uniqueness, $u \equiv v$, and consequently,

$$
T(t) x=\mathcal{C} T(t)^{*} \mathcal{C} x, \quad \forall t \geq 0, \forall x \in \operatorname{dom}(A) .
$$

Since $\operatorname{dom}(A)$ is dense in $\mathcal{H}$, by Lemma 1.1.7, $T(s)$ is $\mathcal{C}$-symmetric, for every $s \geq 0$.

Theorem 3.2 .2 can be extended in the case of groups as follows. 
Corollary 3.2.3. Let $(V(s))_{s \in \mathbb{R}}$ a $C_{0}$-group on a Hilbert space $\mathcal{H}$, with the generator $A$, and $\mathcal{C}: \mathcal{H} \rightarrow \mathcal{H}$ a conjugation. If $A$ is $\mathcal{C}$-selfadjoint, then the operator $V(s)$ is $\mathcal{C}$-symmetric, for every $s \in \mathbb{R}$.

Proof. Clearly, $-A$ is $\mathcal{C}$-selfadjoint, since

$$
(-A)^{*}=-A^{*}=-\mathcal{C} A \mathcal{C}=\mathcal{C}(-A) \mathcal{C} .
$$

As introduced in Section $1.3,\left(V_{+}(s)\right)_{s \geq 0}$ and $\left(V_{-}(s)\right)_{s \geq 0}$ are $C_{0}$-semigroups. Now by Theorem 3.2.2, we have

$$
V(s)^{*}=V_{+}(s)^{*}=\mathcal{C} V_{+}(s) \mathcal{C}=\mathcal{C} V(s) \mathcal{C}, \quad \forall s \geq 0
$$

and

$$
V(-s)^{*}=V_{-}(s)^{*}=\mathcal{C} V_{-}(s) \mathcal{C}=\mathcal{C} V(-s) \mathcal{C}, \quad \forall s \geq 0 .
$$

Thus, $V(s)$ is $\mathcal{C}$-symmetric, for every $s \in \mathbb{R}$.

\subsubsection{Solution to the second question}

The following theorem gives the answer for Question B2.

Theorem 3.2.4 (Stone-type theorem). Let $(T(s))_{s \geq 0}$ be a $C_{0}$-semigroup on a Hilbert space $\mathcal{H}$, with the generator $A$, and $\mathcal{C}: \mathcal{H} \rightarrow \mathcal{H}$ a conjugation. If $T(s)$ is $\mathcal{C}$-symmetric, for every $s \geq 0$, then $A$ is $\mathcal{C}$-selfadjoint.

Proof. We first show that $A$ is $\mathcal{C}$-symmetric. Namely,

$$
\operatorname{dom}(A) \subseteq \operatorname{dom}\left(\mathcal{C} A^{*} \mathcal{C}\right), \quad A y=\mathcal{C} A^{*} \mathcal{C} y, \quad \forall y \in \operatorname{dom}(A)
$$

We take arbitrarily $x, y \in \operatorname{dom}(A)$, and observe that

$$
\begin{aligned}
\left\langle\frac{T(s) x-x}{s}, \mathcal{C} y\right\rangle & =\left\langle\left(\frac{T(s)-I}{s}\right) x, \mathcal{C} y\right\rangle=\left\langle x,\left(\frac{T(s)-I}{s}\right)^{*} \mathcal{C} y\right\rangle \\
& =\left\langle x, \frac{T(s)^{*}-I}{s} \mathcal{C} y\right\rangle=\left\langle x, \frac{T(s)^{*} \mathcal{C} y-\mathcal{C} y}{s}\right\rangle \\
& =\left\langle x, \frac{\mathcal{C} T(s) y-\mathcal{C} y}{s}\right\rangle=\left\langle x, \mathcal{C} \frac{T(s) y-y}{s}\right\rangle .
\end{aligned}
$$


Let $s \rightarrow 0^{+}$in the above identity to get

$$
\langle A x, \mathcal{C} y\rangle=\langle x, \mathcal{C} A y\rangle, \quad \forall x, y \in \operatorname{dom}(A)
$$

By definition of the adjoint operator (see [22, Chapter VIII]), equation (3.1) gives $\mathcal{C} y \in \operatorname{dom}\left(A^{*}\right)$ and $A^{*}(\mathcal{C} y)=\mathcal{C} A y$. So, we have

$$
y \in \operatorname{dom}\left(\mathcal{C} A^{*} \mathcal{C}\right), \mathcal{C} A^{*} \mathcal{C} y=\mathcal{C}^{2} A y=A y
$$

which imply, due to the arbitrariness of $y$, that

$$
A \preceq \mathcal{C} A^{*} \mathcal{C}
$$

For the reverse inclusion, since $T(s)$ is bounded, $T(s)^{* *}=T(s)$ for every $s \geq 0$. Applying the conjugation $\mathcal{C}$ to both sides, we get

$$
\mathcal{C} T(s)^{* *} \mathcal{C}=\mathcal{C} T(s) \mathcal{C}=T(s)^{*}, \quad \forall s \geq 0
$$

The last equality holds because $T(s)$ is $\mathcal{C}$-symmetric. By arguments similar to those used in the first step but now applied to $\left(T(s)^{*}\right)_{s \geq 0}$, we get

$$
A^{*} \preceq \mathcal{C} A^{* *} \mathcal{C}
$$

As mentioned in Section 1.3, the generator of a $C_{0}$-semigroup is always closed and densely defined, and consequently, $A=A^{* *}$ by [22, Theorem VIII.1]. Hence, inclusion 3.2 becomes

$$
A^{*} \preceq \mathcal{C} A^{* *} \mathcal{C}=\mathcal{C} A \mathcal{C}
$$

which implies, by the boundedness of $\mathcal{C}$, that

$$
\mathcal{C} A^{*} \mathcal{C} \preceq \mathcal{C C} A \mathcal{C C}=A \quad(\text { by the involutivity of } \mathcal{C})
$$

As for B1, we can extend Theorem 3.2 .4 to the case of $C_{0}$-groups. 
Corollary 3.2.5. Let $(V(s))_{s \in \mathbb{R}}$ a $C_{0}$-group on a Hilbert space $\mathcal{H}$, with the generator $A$, and $\mathcal{C}: \mathcal{H} \rightarrow \mathcal{H}$ a conjugation. If $V(s)$ is $\mathcal{C}$-symmetric, for every $s \in \mathbb{R}$, then $A$ is $\mathcal{C}$-selfadjoint.

Proof. Since each operator $V(s)$ is $\mathcal{C}$-symmetric, so is $V_{+}(s)$. As mentioned in the Introduction, the operator $A$ generates the $C_{0}$-semigroup $\left(V_{+}(s)\right)_{s \geq 0}$. Now applying Theorem 3.2 .4 to the $C_{0}$-semigroup $\left(V_{+}(s)\right)_{s \geq 0}$, we get the desired result.

\subsection{A new object: $\mathrm{CSO} C_{0}$-semigroups}

Before giving the answer for question B3, we need the results.

Proposition 3.3.1. Let $\mu$ be a finite measure, $(M, \mu)$ a measure space, and $f$ a real-valued function defined on $M$. Let $L^{2}(M, d \mu)$ be the set of equivalence classes of measurable functions $g: M \rightarrow \mathbb{C}$ for which

$$
\|g\|_{L^{2}}=\left(\int_{M}|g(x)|^{2} d \mu(x)\right)^{1 / 2}<\infty .
$$

The following statements hold:

1. The operator $\mathcal{J}$, defined by $\mathcal{J} g(x)=\overline{g(x)}$, is a conjugation on $L^{2}(M, d \mu)$.

2. The operator $M_{f}$, defined by

$$
M_{f}(g)=f g, \quad \operatorname{dom}\left(M_{f}\right)=\left\{g \in L^{2}(M, d \mu): f g \in L^{2}(M, d \mu)\right\},
$$

is $\mathcal{J}$-selfadjoint.

3. Suppose that $\mathcal{H}$ is a Hilbert space. If $U: \mathcal{H} \rightarrow L^{2}(M, d \mu)$ is a unitary linear operator (that is $U^{*} U$ is the identity operator on $L^{2}(M, d \mu)$, while $U U^{*}$ is the identity operator on $\mathcal{H}$ ), then $\mathcal{C}=U^{*} \mathcal{J} U$ is a conjugation on $\mathcal{H}$. 
Proof. Note that $L^{2}(M, d \mu)$ is a Hilbert space with the inner product given by

$$
\langle f, g\rangle_{L^{2}}=\int_{M} f(x) \overline{g(x)} d \mu(x) .
$$

(1) Arbitrarily take $g, h \in L^{2}(M, d \mu)$, and $\alpha, \beta \in \mathbb{C}$. We have

$$
\mathcal{J}(\alpha g+\beta h)(x)=\overline{(\alpha g+\beta h)(x)}=\bar{\alpha} \mathcal{J} g(x)+\bar{\beta} \mathcal{J} h(x),
$$

which implies that $\mathcal{J}$ is anti-linear. Furthermore, the operator $\mathcal{J}$ is involutive, because

$$
\mathcal{J}^{2} g(x)=\overline{\mathcal{J} g(x)}=\overline{\overline{g(x)}}=g(x)
$$

and is isometric, because

$$
\|\mathcal{J} g\|_{L^{2}}^{2}=\int_{\mathbb{R}}|\overline{g(x)}|^{2} d \mu(x)=\int_{\mathbb{R}}|g(x)|^{2} d \mu(x)=\|g\|_{L^{2}}^{2} .
$$

All these arguments show that $\mathcal{J}$ is a conjugation.

(2) We have $\operatorname{dom}\left(\mathcal{J} M_{f} \mathcal{J}\right)=\operatorname{dom}\left(M_{f}\right)$. Indeed,

$$
\begin{aligned}
g \in \operatorname{dom}\left(\mathcal{J} M_{f} \mathcal{J}\right) & \Longleftrightarrow \mathcal{J} g \in \operatorname{dom}\left(M_{f}\right) \Longleftrightarrow \bar{g} \in L^{2}(M, d \mu), f \bar{g} \in L^{2}(M, d \mu) \\
& \Longleftrightarrow g \in L^{2}(M, d \mu), f g \in L^{2}(M, d \mu) \Longleftrightarrow g \in \operatorname{dom}\left(M_{f}\right) .
\end{aligned}
$$

Note that the third equivalence holds, because

$$
\int_{\mathbb{R}}|\overline{g(x)}|^{2} d \mu(x)=\int_{\mathbb{R}}|g(x)|^{2} d \mu(x),
$$

and

$$
\int_{\mathbb{R}}|f(x) \overline{g(x)}|^{2} d \mu(x)=\int_{\mathbb{R}}|f(x) g(x)|^{2} d \mu(x) .
$$

Moreover, we see that

$$
\begin{aligned}
\mathcal{J} M_{f} \mathcal{J} g & =\mathcal{J} M_{f}(\bar{g})=\mathcal{J}(f \bar{g})=\overline{f \bar{g}} \\
& =f g \quad(\text { since } f: M \rightarrow \mathbb{R}) \\
& =M_{f} g, \quad \forall g \in \operatorname{dom}\left(M_{f}\right)
\end{aligned}
$$


All these arguments show that

$$
\mathcal{J} M_{f} \mathcal{J}=M_{f}
$$

Note that by [22, Proposition 1, p. 259], $M_{f}$ is selfadjoint. These give

$$
M_{f}^{*}=M_{f}=\mathcal{J} M_{f} \mathcal{J} .
$$

(3) Clearly, $\mathcal{C}$ is anti-linear. Furthermore, the operator $\mathcal{C}$ is involutive, since

$$
\begin{aligned}
\mathcal{C}^{2} & =U^{*} \mathcal{J} U U^{*} \mathcal{J} U=U^{*} \mathcal{J} \mathcal{J} U \quad \text { (since } U \text { is unitary) } \\
& =U^{*} U \quad \text { (since } \mathcal{J} \text { is a conjugation) } \\
& =I,
\end{aligned}
$$

and is isometric, because

$$
\|\mathcal{C} f\|=\left\|U^{*} \mathcal{J} U f\right\|=\|\mathcal{J} U f\|=\|U f\|=\|f\| .
$$

Proposition 3.3.2. If the operator $A: \operatorname{dom}(A) \subseteq \mathcal{H} \rightarrow \mathcal{H}$ is selfadjoint, then there always exists a conjugation $\mathcal{C}$ such that $A$ is $\mathcal{C}$-selfadjoint.

Proof. Since the operator $A$ is selfadjoint, by [22, Theorem VIII.4], there exist a finite measure $\mu$, a measure space $(M, \mu)$, a unitary operator $U: \mathcal{H} \rightarrow$ $L^{2}(M, d \mu)$, and a function $f: M \rightarrow \mathbb{R}$, such that $U A=M_{f} U$, where the operator $M_{f}: L^{2}(M, d \mu) \rightarrow L^{2}(M, d \mu)$ is defined by

$$
M_{f}(g)=f g,
$$

with the domain

$$
\operatorname{dom}\left(M_{f}\right)=\left\{g \in L^{2}(M, d \mu): f g \in L^{2}(M, d \mu)\right\}
$$


By Proposition 3.3.1, $M_{f}$ is $\mathcal{J}$-selfadjoint. Since $U A=M_{f} U$, taking the adjoint operation both sides, we have

$$
U^{*} M_{f}^{*} \preceq\left(M_{f} U\right)^{*}=(U A)^{*}=A^{*} U^{*} \quad \text { (by [24, Proposition 1.7]). }
$$

Due to the $\mathcal{J}$-selfadjointness of $M_{f}$, we get

$$
U^{*} \mathcal{J} M_{f} \mathcal{J}=U^{*} M_{f}^{*} \preceq A^{*} U^{*}
$$

Again since $M_{f}=U A U^{*}$, we have

$$
U^{*} \mathcal{J} U A U^{*} \mathcal{J} \preceq A^{*} U^{*},
$$

which implies, due to the boundedness of $U$, that

$$
U^{*} \mathcal{J} U A U^{*} \mathcal{J} U \preceq A^{*} U^{*} U=A^{*} .
$$

Setting $\mathcal{C}:=U^{*} \mathcal{J} U$, we obtain $\mathcal{C} A \mathcal{C} \preceq A^{*}$.

For obtaining the reverse inclusion, as $A$ is selfadjoint, we can write

$$
\mathcal{C} A^{*} \mathcal{C}=\mathcal{C} A \mathcal{C} \preceq A^{*}=A,
$$

which gives

$$
A^{*}=\mathcal{C C} A^{*} \mathcal{C C} \preceq \mathcal{C} A \mathcal{C}
$$

By Proposition 3.3.1, $\mathcal{C}$ is a conjugation, and hence, $A$ is $\mathcal{C}$-selfadjoint.

Now we can give an answer for Question B3.

Theorem 3.3.3. Let $(U(t))_{t \in \mathbb{R}}$ be a unitary group. Then we can find a conjugation $\mathcal{C}$ (independent of $t$ ) such that $U(t)^{*}=\mathcal{C} U(t) \mathcal{C}, \forall t \in \mathbb{R}$.

Proof. By Stone's theorem, there exists a unique selfadjoint operator $A$ such that the operator $i A$ generates $(U(t))_{t \in \mathbb{R}}$. By Proposition 3.3.2, there exists a conjugation $\mathcal{C}$ such that $A=\mathcal{C} A^{*} \mathcal{C}$. 
Multiply both sides by $i$ to get

$$
\begin{aligned}
i A & =i \mathcal{C} A^{*} \mathcal{C}=\mathcal{C}\left(-i A^{*}\right) \mathcal{C} \quad(\text { since } \mathcal{C} \text { is anti-linear }) \\
& =\mathcal{C}(i A)^{*} \mathcal{C} \quad(\text { since } \bar{i}=-i)
\end{aligned}
$$

The last equality shows that $i A$ is $\mathcal{C}$-selfadjoint. By Corollary 3.2 .3 , the operators $U(s), s \geq 0$ are $\mathcal{C}$-symmetric.

Inspired by Theorem 3.3.3, we introduce a new concept in dynamical systems, which is related closely to complex symmetric operators.

Definition 3.3.4. Let $(T(s))_{s \geq 0}$ be a $C_{0}$-semigroup on a Hilbert space $\mathcal{H}$, and $\mathcal{C}: \mathcal{H} \rightarrow \mathcal{H}$ a conjugation (independent of $s$ ). We say that $(T(s))_{s \geq 0}$ is a $\mathcal{C}$ symmetric $C_{0}$-semigroup if

$$
T(t)=\mathcal{C} T(t)^{*} \mathcal{C}, \quad \forall t \geq 0
$$

In this case, $(T(s))_{s \geq 0}$ is called a complex symmetric $C_{0}$-semigroup. Sometimes, we write briefly as $C S O C_{0}$-semigroup.

Likewise, extending this definition to $t \in \mathbb{R}$, we get a $\mathcal{C}$-symmetric $C_{0}$-group .

Note that if an operator $A$ is bounded and complex symmetric, then the corresponding semigroup is defined by $e^{t A}$.

\subsection{Semigroup of weighted composition opera- tors}

To construct complex symmetric $C_{0}$-semigroups on a Hilbert space of holomorphic functions, we make use of semigroups of weighted composition operators. The concepts of semiflows and semicocycles are among the most important features in defining these semigroups. 
Definition 3.4.1. A family $\left(\phi_{t}\right)_{t \geq 0}$ of non-constant holomorphic functions on a domain $G$ is called a semiflow if

1. $\phi_{t}: G \rightarrow G, \forall t \geq 0$;

2. $\phi_{0}(z)=z, \forall z \in G$;

3. $\phi_{t+s}(z)=\phi_{t}\left(\phi_{s}(z)\right), \forall t, s \geq 0, \forall z \in G$.

Likewise, if $t, s \in \mathbb{R}$, then it is called a flow.

A trivial example of a semiflow is $\phi_{t}(z)=z, \forall t \geq 0$. In the case that $G$ is the whole complex plane, the structure of semiflows can be given as follows.

Proposition 3.4.2 ([14]). If $\left(\phi_{t}\right)_{t \geq 0}$ is a non-trivial semiflow on $\mathbb{C}$, then it has at most one fixed point and one of the following statements holds:

1. If it has no fixed point in $\mathbb{C}$, then there exists a complex constant $K \neq 0$ such that $\phi_{t}(z)=z+K t$.

2. If it has one fixed point in $\mathbb{C}$, then there exist complex constants $K$ and $\alpha \neq 0$ such that $\phi_{t}(z)=e^{t \alpha} z+K\left(1-e^{t \alpha}\right)$.

Definition 3.4.3. Let $\left(\phi_{t}\right)_{t \geq 0}$ be a semiflow. A family $\left(h_{t}\right)_{t \geq 0}$ of holomorphic functions on a domain $G$ is called a semicocycle for $\left(\phi_{t}\right)_{t \geq 0}$ if

1. For every $z \in G$, the mapping $t \mapsto h_{t}(z)$ is differentiable;

2. $h_{t+s}=h_{t} \cdot\left(h_{s} \circ \phi_{t}\right), \forall t, s \geq 0$;

3. $h_{0}(z)=1, \forall z \in G$.

Let $\left(\phi_{t}\right)_{t \geq 0}$ be a semiflow, $\left(h_{t}\right)_{t \geq 0}$ its corresponding semicocycle, and let furthermore $\mathcal{H}$ be a Hilbert space of holomorphic functions on a domain $G$. We consider the family $(\mathcal{T}(t))_{t \geq 0}$ of weighted composition operators defined by

$$
(\mathcal{T}(t) f)(z):=h_{t}(z) f\left(\phi_{t}(z)\right), z \in \mathbb{C}
$$


We prove the following observation:

Proposition 3.4.4. The family $(\mathcal{T}(t))_{t \geq 0}$ satisfies axioms (1)-(2) of Definition 1.3.1. Namely,

$$
\mathcal{T}(0)=I, \quad \mathcal{T}(t) \mathcal{T}(s)=\mathcal{T}(t+s), \forall t, s \geq 0
$$

Proof. Indeed, we have

$$
\mathcal{T}(0) f(z)=h_{0}(z) f\left(\phi_{0}(z)\right)=f(z), \quad \forall z \in G, \forall f \in \mathcal{H}
$$

Furthermore, by (3.3),

$$
\begin{aligned}
\mathcal{T}(t) \mathcal{T}(s) f(z) & =\mathcal{T}(t)(\mathcal{T}(s) f)(z)=h_{t}(z) h_{s}\left(\phi_{t}(z)\right) f\left(\phi_{s}\left(\phi_{t}(z)\right)\right) \\
& =h_{t+s}(z) f\left(\phi_{t+s}(z)\right), \quad \forall z \in G, \forall f \in \mathcal{H} .
\end{aligned}
$$

Although the family $(\mathcal{T}(t))_{t \geq 0}$ satisfies axioms (1)-(2), in general, it is not a semigroup under terms of Definition 1.3.1 (because it may be unbounded). But the term "semigroup of weighted composition operators" is used in this thesis to indicate the family defined by (3.3). The survey [25] is a good source providing more information on these semigroups when $G=\mathbb{D}$, the unit disk.

To find conditions under which $(\mathcal{T}(t))_{t \geq 0}$ is a complex symmetric $C_{0}$-semigroup, we take turns answering the following questions:

C1. When are the operators $\mathcal{T}(t), t \geq 0$, bounded on $\mathcal{H}$ ?

C2. When are the operators $\mathcal{T}(t), t \geq 0$, complex symmetric on $\mathcal{H}$ ?

C3. When is the family $(\mathcal{T}(t))_{t \geq 0}$ strongly continuous on $\mathcal{H}$ ?

Note that for each $t \geq 0$, the operator $\mathcal{T}(t)$ is a weighted composition operator, which is induced by $h_{t}($.$) and \phi_{t}($.$) . This means that question \mathbf{C 1}$ is reduced to 
studying the boundedness of weighted composition operators on function spaces, while question $\mathbf{C 2}$ is simplified to investigating question $\mathbf{A}$.

Note that under additional assumption of the density of polynomials, we can reduce question $\mathbf{C 3}$ to the problem of checking the following limit

$$
\lim _{t \rightarrow 0^{+}}\|\mathcal{T}(t) P-P\|=0
$$

where $P$ is an arbitrary polynomial.

\section{$3.5 \mathcal{C}_{a, b, c^{-}}$-symmetric $C_{0}$-semigroups on $\mathcal{F}^{2}(\mathbb{C})$}

In this section, we characterize the structure of the family $(\mathcal{T}(t))_{t \geq 0}$, when it is a $\mathcal{C}_{a, b, c}$-symmetric $C_{0}$-semigroup on $\mathcal{F}^{2}(\mathbb{C})$.

Recall that $(\mathcal{T}(t))_{t \geq 0}$ is a semigroup of weighted composition operators on $\mathcal{F}^{2}(\mathbb{C})$ defined by (3.3), that is

$$
(\mathcal{T}(t) f)(z)=h_{t}(z) f\left(\phi_{t}(z)\right), f \in \mathcal{F}^{2}(\mathbb{C}), z \in \mathbb{C}
$$

where $\left(\phi_{t}\right)_{t \geq 0}$ is a semiflow with the corresponding semicocycle $\left(h_{t}\right)_{t \geq 0}$.

The lemma below is useful for obtaining the explicit formula of $(\mathcal{T}(t))_{t \geq 0}$.

Lemma 3.5.1. Suppose that the differentiable function $E: \mathbb{R}_{+} \rightarrow \mathbb{C}$ satisfy

$$
\left\{\begin{array}{l}
E(t+s)=E(t) E(s) e^{F(t, s)}, \forall t, s \geq 0, \\
E(0)=1, F(t, 0)=0
\end{array}\right.
$$

where $F: \mathbb{R}_{+} \times \mathbb{R}_{+} \rightarrow \mathbb{C}$ is a differentiable function of two variables. Then the function $E(t)$ must be of the form

$$
E(t)=\exp \left(t G+\int_{0}^{t} \frac{\partial F}{\partial s}(\tau, 0) d \tau\right)
$$

where $G=E^{\prime}(0)$. In particular, 
1. If $F(t, s)=\theta t s$, then $E(t)=e^{t G+\theta t^{2} / 2}$.

2. If $F(t, s)=\theta\left(1-e^{\alpha s}\right)\left(1-e^{\alpha t}\right)$, then $E(t)=e^{G t+\theta\left(e^{\alpha t}-\alpha t-1\right)}$.

3. If $F(t, s)=0$, then $E(t)=e^{t G}$.

4. If $F(t, s)=\theta(\sin (t+s) \lambda-\sin t \lambda-\sin s \lambda)$, then $E(t)=e^{t G+\theta(\sin t \lambda-t \lambda)}$.

Proof. We can rewrite

$$
\frac{E(t+s)-E(t)}{s}=E(t)\left(\frac{E(s)-1}{s}\right) e^{F(t, s)}+E(t)\left(\frac{e^{F(t, s)}-e^{F(t, 0)}}{s}\right) .
$$

Letting $s \rightarrow 0$ gives

$$
\begin{aligned}
E^{\prime}(t) & =E(t) E^{\prime}(0) e^{F(t, 0)}+E(t) \frac{\partial F}{\partial s}(t, 0) e^{F(t, 0)} \\
& =E(t)\left(E^{\prime}(0)+\frac{\partial F}{\partial s}(t, 0)\right) \quad(\text { since } F(t, 0)=0)
\end{aligned}
$$

Putting

$$
G(t)=E(t) \exp \left(-t E^{\prime}(0)-\int_{0}^{t} \frac{\partial F}{\partial s}(\tau, 0) d \tau\right)
$$

we have $G(0)=E(0)=1$, and furthermore,

$$
\begin{aligned}
E^{\prime}(t)= & \left(G(t) \exp \left(t E^{\prime}(0)+\int_{0}^{t} \frac{\partial F}{\partial s}(\tau, 0) d \tau\right)\right)^{\prime} \\
= & G^{\prime}(t) \exp \left(t E^{\prime}(0)+\int_{0}^{t} \frac{\partial F}{\partial s}(\tau, 0) d \tau\right) \\
& +G(t)\left(E^{\prime}(0)+\frac{\partial F}{\partial s}(t, 0)\right) \exp \left(t E^{\prime}(0)+\int_{0}^{t} \frac{\partial F}{\partial s}(\tau, 0) d \tau\right) \\
= & G^{\prime}(t) \exp \left(t E^{\prime}(0)+\int_{0}^{t} \frac{\partial F}{\partial s}(\tau, 0) d \tau\right)+E(t)\left(E^{\prime}(0)+\frac{\partial F}{\partial s}(t, 0)\right) .
\end{aligned}
$$

Hence, equation (3.4) is reduced to

$$
G^{\prime}(t) \exp \left(t E^{\prime}(0)+\int_{0}^{t} \frac{\partial F}{\partial s}(\tau, 0) d \tau\right)=0 \Longleftrightarrow G(t)=G(0)=1 .
$$

Substituting $G$ back into $F$, we get the desired form. 
(1) If $F(t, s)=\theta t s$, then $\frac{\partial F}{\partial s}(t, s)=\theta t$ and hence,

$$
\int_{0}^{t} \frac{\partial F}{\partial s}(\tau, 0) d \tau=\frac{\theta t^{2}}{2}
$$

(2) If $F(t, s)=\theta\left(1-e^{\alpha s}\right)\left(1-e^{\alpha t}\right)$, then

$$
\frac{\partial F}{\partial s}(t, s)=-\alpha \theta e^{\alpha s}\left(1-e^{\alpha t}\right)
$$

Therefore,

$$
\int_{0}^{t} \frac{\partial F}{\partial s}(\tau, 0) d \tau=-\alpha \theta \int_{0}^{t}\left(1-e^{\alpha \tau}\right) d \tau=-\theta\left(1+\alpha t-e^{\alpha t}\right) .
$$

(3) This is an immediate consequence of conclusion (2) when $\theta=0$.

(4) If $F(t, s)=\theta(\sin (t+s) \lambda-\sin t \lambda-\sin s \lambda)$, then

$$
\frac{\partial F}{\partial s}(t, s)=\theta \lambda(\cos (t+s) \lambda-\cos s \lambda)
$$

Thus,

$$
\int_{0}^{t} \frac{\partial F}{\partial s}(\tau, 0) d \tau=\int_{0}^{t} \theta \lambda(\cos \tau \lambda-1) d \tau=\theta(\sin t \lambda-t \lambda)
$$

\subsubsection{A strong continuity of semigroups of weighted com- position operators}

By Theorem 2.2.8, for each $t \geq 0, h_{t}(z)$ must be an exponential function $C_{t} e^{D_{t} z}$ whenever $\mathcal{T}(t)$ is $\mathcal{C}_{a, b, c}$-symmetric. Thus, it is essential to study a strong continuity of the family $(\mathcal{T}(t))_{t \geq 0}$ with such functions $h_{t}(),. t \geq 0$.

Proposition 3.5.2. Let $\left(\phi_{t}\right)_{t \geq 0}$ be a semiflow on $\mathbb{C}$, such that its corresponding semicocycle has the form $h_{t}(z)=C_{t} e^{D_{t} z}$, with conditions

$$
\lim _{t \rightarrow 0^{+}} C_{t}=1, \quad \lim _{t \rightarrow 0^{+}} D_{t}=0 .
$$

Suppose that the operator $\mathcal{T}(t)$ is bounded, for each $t \geq 0$. Then 
1. $(\mathcal{T}(t))_{t \geq 0}$ is strongly continuous with respect to polynomials, i.e.

$$
\lim _{t \rightarrow 0^{+}}\|\mathcal{T}(t) P-P\|=0, \quad \text { for every polynomial } P
$$

2. $(\mathcal{T}(t))_{t \geq 0}$ is a $C_{0}$-semigroup if and only if there exist $\delta>0$ and $\Delta=\Delta(\delta)>0$, such that for every $t \in[0, \delta]$ we always have $\|\mathcal{T}(t)\|<\Delta$.

Proof. Clearly, conclusion (2) can be obtained from conclusion (1) by applying [4, Proposition I.5.3].

We prove $(1)$. As $\left(z^{m}\right)$ is a basis for $\mathcal{F}^{2}(\mathbb{C})$, it suffices to prove conclusion (1) for each element $P_{m}(z)=z^{m}$. Indeed, for each $P_{m}$, we can write

$$
\begin{aligned}
\mathcal{T}(t) P_{m}(z)-P_{m}(z)= & \left(C_{t}-1\right) P_{m}(z)+C_{t}\left(e^{D_{t} z}-1\right) P_{m}(z) \\
& +h_{t}(z)\left(\phi_{t}(z)^{m}-P_{m}(z)\right) .
\end{aligned}
$$

It makes sense to consider the functions

$$
R_{t}(z)=C_{t}\left(e^{D_{t} z}-1\right) P_{m}(z), \quad Q_{t}(z)=h_{t}(z)\left(\phi_{t}(z)^{m}-P_{m}(z)\right) .
$$

- Note that the first term tends to 0 in $\mathcal{F}^{2}(\mathbb{C})$ as $t \rightarrow 0^{+}$because

$$
\lim _{t \rightarrow 0^{+}}\left\|\left(C_{t}-1\right) P_{m}\right\|=\sqrt{m !} \lim _{t \rightarrow 0^{+}}\left|C_{t}-1\right|=0 .
$$

By (3.5), we can find $\delta \in(0,1)$ such that

$$
\sup _{0 \leq t \leq \delta}\left|C_{t}\right| \leq 2, \quad \sup _{0 \leq t \leq \delta}\left|D_{t}\right| \leq 1 .
$$

In the rest of the proof, we always consider $t \in[0, \delta]$.

- Clearly, $R_{t}$ is an entire function, whose Taylor series is

$$
R_{t}(z)=C_{t} \sum_{k \geq 1} \frac{\left(D_{t} z\right)^{k}}{k !} P_{m}(z)=C_{t} \sum_{k \geq 1} \frac{D_{t}^{k}}{k !} P_{m+k}(z), z \in \mathbb{C} .
$$


The above expansion allows us to show that $R_{t} \in \mathcal{F}^{2}(\mathbb{C})$. Indeed, since $\left|C_{t}\right| \leq 2$ and $\left|D_{t}\right| \leq 1$, we have

$$
\begin{aligned}
\left|C_{t}\right|^{2} \sum_{k \geq 1} \frac{\left|D_{t}\right|^{2 k}}{(k !)^{2}}\left\|P_{m+k}\right\|^{2} & =\left|C_{t}\right|^{2} \sum_{k \geq 1} \frac{\left|D_{t}\right|^{2 k}(m+k) !}{(k !)^{2}} \leq 4 \sum_{k \geq 1} \frac{\left|D_{t}\right|^{2 k}(m+k) !}{(k !)^{2}} \\
& =4\left|D_{t}\right| \sum_{k \geq 1} \frac{\left|D_{t}\right|^{2 k-1}(m+k) !}{(k !)^{2}} \leq 4\left|D_{t}\right| \sum_{k \geq 1} \frac{(m+k) !}{(k !)^{2}} .
\end{aligned}
$$

By the D'Alembert test, the right-hand side is always finite. Hence, $R_{t} \in \mathcal{F}^{2}(\mathbb{C})$, and moreover, its norm satisfies

$$
\left\|R_{t}\right\|^{2}=\left|C_{t}\right|^{2} \sum_{k \geq 1} \frac{\left|D_{t}\right|^{2 k}}{(k !)^{2}}\left\|P_{m+k}\right\|^{2} \leq 4\left|D_{t}\right| \sum_{k \geq 1} \frac{(m+k) !}{(k !)^{2}} .
$$

Again by 3.5$), R_{t}$ converges to 0 in the $\mathcal{F}^{2}(\mathbb{C})$-norm as $t \rightarrow 0^{+}$.

- Finally, we prove that $\lim _{t \rightarrow 0^{+}} Q_{t}=0$ in the $\mathcal{F}^{2}(\mathbb{C})$-norm.

Note that

$$
\begin{aligned}
\left\|Q_{t}\right\|^{2} & =\frac{1}{\pi} \int_{\mathbb{C}}\left|h_{t}(z)\left(\phi_{t}(z)^{m}-P_{m}(z)\right)\right|^{2} e^{-|z|^{2}} d V(z) \\
& =\frac{1}{\pi} \int_{\mathbb{C}}\left|C_{t}\right|^{2}\left|\phi_{t}(z)^{m}-P_{m}(z)\right|^{2} e^{2 \operatorname{Re}\left(D_{t} z\right)-|z|^{2}} d V(z) .
\end{aligned}
$$

Since $\left|C_{t}\right| \leq 2$, we see

$$
\begin{aligned}
\left\|Q_{t}\right\|^{2} & \leq \frac{4}{\pi} \int_{\mathbb{C}}\left|\phi_{t}(z)^{m}-z^{m}\right|^{2} e^{2 \operatorname{Re}\left(D_{t} z\right)-|z|^{2}} d V(z) \\
& \leq \frac{4}{\pi} \int_{\mathbb{C}}\left|\phi_{t}(z)^{m}-z^{m}\right|^{2} e^{2\left|D_{t} z\right|-|z|^{2}} d V(z) \\
& \leq \frac{4}{\pi} \int_{\mathbb{C}}\left|\phi_{t}(z)^{m}-z^{m}\right|^{2} e^{2|z|-|z|^{2}} d V(z) .
\end{aligned}
$$

In view of Proposition 3.4.2, we consider two cases of the semiflow $\left(\phi_{t}\right)$ to estimate the expression $\left|\phi_{t}(z)^{m}-z^{m}\right|$.

Case 1: $\left(\phi_{t}\right)_{t \geq 0}$ has no fixed point in $\mathbb{C}$. In this case, $\phi_{t}(z)=z+K t$ for some $K \in \mathbb{C} \backslash\{0\}$. Then we have

$$
\left|\phi_{t}(z)^{m}-z^{m}\right|=\left|\sum_{k=1}^{m}\left(\begin{array}{c}
m \\
k
\end{array}\right) z^{m-k}(K t)^{k}\right| \leq L_{1} \sum_{k=1}^{m}|z|^{m-k} t^{k},
$$


where

$$
L_{1}:=\max _{1 \leq k \leq m}\left(\begin{array}{c}
m \\
k
\end{array}\right)|K|^{k} .
$$

Hence, by the Cauchy-Schwarz inequality, we have

$$
\left|\phi_{t}(z)^{m}-z^{m}\right|^{2} \leq\left(L_{1}\right)^{2} m \sum_{k=1}^{m}|z|^{2(m-k)} t^{2 k}
$$

which gives

$$
\begin{aligned}
\left\|Q_{t}\right\|^{2} & \leq \frac{4\left(L_{1}\right)^{2} m}{\pi} \sum_{k=1}^{m} t^{2 k} \int_{\mathbb{C}}|z|^{2(m-k)} e^{2|z|-|z|^{2}} d V(z) \\
& <\infty \quad \text { (by Lemma 2.2.9. }
\end{aligned}
$$

This shows that $Q_{t} \in \mathcal{F}^{2}(\mathbb{C})$, and moreover, its norm satisfies (3.7). Clearly, $Q_{t}$ must converge to 0 in $\mathcal{F}^{2}(\mathbb{C})$ as $t \rightarrow 0^{+}$.

Case 2: $\left(\phi_{t}\right)_{t \geq 0}$ has a fixed point in $\mathbb{C}$. In this case, $\phi_{t}(z)=e^{t \alpha} z+K\left(1-e^{t \alpha}\right)$ for some $K \in \mathbb{C}$ and $\alpha \in \mathbb{C} \backslash\{0\}$. Then

$$
\phi_{t}(z)^{m}-z^{m}=\left(e^{t m \alpha}-1\right) z^{m}+\sum_{k=1}^{m}\left(\begin{array}{c}
m \\
k
\end{array}\right) e^{t(m-k) \alpha} z^{m-k} K^{k}\left(1-e^{t \alpha}\right)^{k} .
$$

Since

$$
\left|e^{t(m-k) \alpha}\right| \leq e^{(m-k)|\operatorname{Re} \alpha|},
$$

we estimate

$$
\left|\phi_{t}(z)^{m}-z^{m}\right| \leq L_{2}\left|e^{t m \alpha}-1\right||z|^{m}+L_{2} \sum_{k=1}^{m}\left|e^{t \alpha}-1\right|^{k}|z|^{m-k},
$$

where

$$
L_{2}=\max _{1 \leq k \leq m}\left\{1,\left(\begin{array}{c}
m \\
k
\end{array}\right) e^{(m-k)|\operatorname{Re} \alpha|}|K|^{k}\right\} .
$$

Again by the Cauchy-Schwarz inequality, we have

$$
\left|\phi_{t}(z)^{m}-z^{m}\right|^{2} \leq\left(L_{2}\right)^{2}(m+1)\left(\left|e^{t m \alpha}-1\right|^{2}|z|^{2 m}+\sum_{k=1}^{m}\left|e^{t \alpha}-1\right|^{2 k}|z|^{2(m-k)}\right),
$$


which gives

$$
\begin{aligned}
\left\|Q_{t}\right\|^{2} \leq & \frac{4}{\pi}\left(L_{2}\right)^{2}(m+1)\left|e^{t m \alpha}-1\right|^{2} \int_{\mathbb{C}}|z|^{2 m} e^{2|z|-|z|^{2}} d V(z) \\
& +\frac{4}{\pi}\left(L_{2}\right)^{2}(m+1) \sum_{k=1}^{m}\left|e^{t \alpha}-1\right|^{2 k} \int_{\mathbb{C}}|z|^{2(m-k)} e^{2|z|-|z|^{2}} d V(z) \\
< & \infty \quad \text { by Lemma } 2.2 .9 .
\end{aligned}
$$

This shows that $Q_{t} \in \mathcal{F}^{2}(\mathbb{C})$, and moreover, its norm satisfies (3.8). Clearly, $Q_{t}$ must converge to 0 in $\mathcal{F}^{2}(\mathbb{C})$ as $t \rightarrow 0^{+}$. The proof is complete.

Recall that if the operators of the family $(\mathcal{T}(t))_{t \geq 0}$ are $\mathcal{C}_{a, b, c}$-symmetric, then by Theorem 2.2.8, $h_{t}(z)=C_{t} e^{D_{t} z}$, where functions $C_{t}$ and $D_{t}$ satisfy condition (3.5). This note allows us to give the structure of the family $(\mathcal{T}(t))_{t \geq 0}$, when it is a $\mathcal{C}_{a, b, c}$-symmetric $C_{0}$-semigroup on the Fock space $\mathcal{F}^{2}(\mathbb{C})$.

Proposition 3.5.3. Let $(\mathcal{T}(t))_{t \geq 0}$ be a semigroup of weighted composition operators induced by the semiflow $\left(\phi_{t}\right)_{t \geq 0}$ and the corresponding semicocycle $\left(h_{t}\right)_{t \geq 0}$. Suppose that $\mathcal{T}(t)$ is $\mathcal{C}_{a, b, c}$-symmetric and bounded on $\mathcal{F}^{2}(\mathbb{C})$, for every $t \geq 0$. The following statements hold.

1. If $\left(\phi_{t}\right)_{t \geq 0}$ has no fixed point, then there exist complex constants $K \neq 0, G$, such that

$$
\left\{\begin{array}{l}
\bar{K}+a K=0 \\
\phi_{t}(z)=z+K t, h_{t}(z)=e^{G t+a K^{2} t^{2} / 2+a K t z} .
\end{array}\right.
$$

2. If $\left(\phi_{t}\right)_{t \geq 0}$ has one fixed point, then there exist complex constants $G, K$, and $\alpha \neq 0$, such that

$$
\left\{\begin{array}{l}
\text { either } \operatorname{Re} \alpha<0, \text { or } \operatorname{Re} \alpha=0, a K+b-\bar{K}=0 \\
\phi_{t}(z)=e^{t \alpha} z+K\left(1-e^{t \alpha}\right) \\
h_{t}(z)=e^{G t+K(a K+b)\left(e^{\alpha t}-\alpha t-1\right)+(a K+b)\left(1-e^{\alpha t}\right) z}
\end{array}\right.
$$




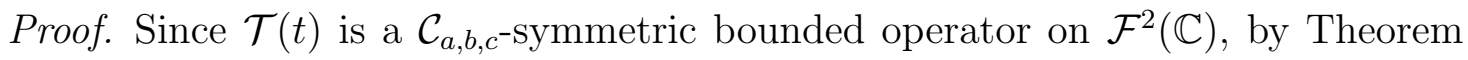
2.2 .8

$$
\phi_{t}(z)=A_{t} z+B_{t}, \quad h_{t}(z)=C_{t} e^{D_{t} z}
$$

where $C_{t} \neq 0, D_{t}=a B_{t}+b\left(1-A_{t}\right)$, and $\left|A_{t}\right| \leq 1$. Note that coefficients $A_{t}, B_{t}, C_{t}$ are complex-valued functions of one variable $t$. We have

$$
\begin{aligned}
h_{t}(z) h_{s}\left(\phi_{t}(z)\right) & =C_{t} e^{z D_{t}} C_{s} e^{\phi_{t}(z) D_{s}}=C_{t} e^{z D_{t}} C_{s} e^{\left(z A_{t}+B_{t}\right) D_{s}} \\
& =C_{t} C_{s} e^{B_{t} D_{s}+z\left(D_{t}+A_{t} D_{s}\right)},
\end{aligned}
$$

which implies, as $h_{t+s}=h_{t} \cdot h_{s} \circ \phi_{t}$, that

$$
C_{t+s} e^{z D_{t+s}}=C_{t} C_{s} e^{B_{t} D_{s}+z\left(D_{t}+A_{t} D_{s}\right)}, \quad \forall z \in \mathbb{C}, \forall t, s \geq 0
$$

In particular, for $z=0$, we get

$$
C_{t+s}=C_{t} C_{s} e^{B_{t} D_{s}}, \quad \forall t, s \geq 0
$$

(1) If $\left(\phi_{t}\right)_{t \geq 0}$ has no fixed point, then $\phi_{t}(z)=z+K t$ for some $K \in \mathbb{C} \backslash\{0\}$. In this case,

$$
A_{t} \equiv 1, \quad B_{t}=K t
$$

and hence, $D_{t}=a K t$. Now we apply Lemma 3.5.1(1) to equation (3.11) to get

$$
C_{t}=e^{G t+a K^{2} t^{2} / 2}
$$

Furthermore, since the coefficient of $z$ in $\phi_{t}(z)$ is $1, B_{t}$ and $D_{t}$ must satisfy condition (3b) of Proposition 2.1.13, which gives $\bar{K}+a K=0$.

(2) If $\left(\phi_{t}\right)_{t \geq 0}$ has one fixed point, then we have $\phi_{t}(z)=e^{t \alpha} z+K\left(1-e^{t \alpha}\right)$, for some $K \in \mathbb{C}$ and $\alpha \in \mathbb{C} \backslash\{0\}$. In this case,

$$
A_{t}=e^{t \alpha}, \quad B_{t}=K\left(1-e^{t \alpha}\right)
$$


and hence, $D_{t}=(a K+b)\left(1-e^{t \alpha}\right)$. We again apply Lemma 3.5.1 (1) to equation (3.11) to get

$$
C_{t}=e^{G t+K(a K+b)\left(e^{\alpha t}-\alpha t-1\right)} .
$$

Furthermore, since $\left|A_{t}\right| \leq 1, \operatorname{Re} \alpha \leq 0$. In case $\operatorname{Re} \alpha=0$, we have $\left|A_{t}\right|=1$ and hence, condition (3b) of Proposition 2.1 .13 is reduced to $a K+b-\bar{K}=0$.

It turns out that the $\mathcal{C}_{a, b, c}$-symmetry of the family $(\mathcal{T}(t))_{t \geq 0}$ is a sufficient condition for the strong continuity.

Proposition 3.5.4. Every semigroup $(\mathcal{T}(t))_{t \geq 0}$ of $\mathcal{C}_{a, b, c}$-symmetric bounded weighted composition operators on $\mathcal{F}^{2}(\mathbb{C})$ is strongly continuous.

Proof. Before proving, we mention a useful remark, which is obtained from 2.2 : If the coefficient of $z$ in the form of $\phi_{t}$ has modulus 1 (that is $\phi_{t}(z)=A_{t} z+B_{t}$ with $\left.\left|A_{t}\right|=1\right)$, then $\|\mathcal{T}(t)\|=\sqrt{M\left(h_{t}, \phi_{t}\right)}$. By Proposition 3.5.3, we have to consider two cases for $\left(\phi_{t}\right)_{t \geq 0}$.

- If $\left(\phi_{t}\right)_{t \geq 0}$ has no fixed point, then by Proposition 3.5.3 (1), we have (3.9). In this case,

$$
A_{t} \equiv 1, B_{t}=K t, C_{t}=e^{t G+a K^{2} t^{2} / 2}, D_{t}=a K t
$$

Since $A_{t}=1$ for each $t \geq 0,\|\mathcal{T}(t)\|=\sqrt{M\left(h_{t}, \phi_{t}\right)}$. Moreover, by (2.6), we have

$$
\begin{aligned}
M\left(h_{t}, \phi_{t}\right) & =\left|C_{t}\right|^{2} e^{\left|B_{t}\right|^{2}}=e^{2 t \operatorname{Re} G+t^{2} \operatorname{Re}\left(a K^{2}\right)+|K|^{2} t^{2}} \\
& =e^{2 t \operatorname{Re} G+t^{2} \operatorname{Re}\left(a K^{2}+|K|^{2}\right)}=e^{2 t \operatorname{Re} G+t^{2} \operatorname{Re}[K(a K+\bar{K})]} \\
& \left.=e^{2 t \operatorname{Re} G} \quad \text { (because } \bar{K}+a K=0\right) .
\end{aligned}
$$

Consequently,

$$
\sup _{0 \leq t \leq 1}\|\mathcal{T}(t)\| \leq e^{|\operatorname{Re} G|}
$$

and hence, since $C_{t}=e^{G t+a K^{2} t^{2} / 2}$ and $D_{t}=a K t$ satisfy condition (3.5), by Proposition $3.5 .2(2),(\mathcal{T}(t))_{t \geq 0}$ is a $C_{0}$-semigroup. 
- If $\left(\phi_{t}\right)_{t \geq 0}$ has a fixed point, then by Proposition 3.5.3 (2), we have 3.10). In this case,

$$
A_{t}=e^{t \alpha}, B_{t}=K\left(1-e^{t \alpha}\right), C_{t}=e^{G t+K(a K+b)\left(e^{\alpha t}-\alpha t-1\right)}, D_{t}=(a K+b)\left(1-e^{t \alpha}\right) .
$$

Now take $\delta \in(0,1)$ such that

$$
\sup _{0 \leq t \leq \delta}\left|C_{t}\right|<2, \quad \sup _{0 \leq t \leq \delta}\left|B_{t}\right|<1
$$

and

$$
\sup _{0 \leq t \leq \delta}\left|\frac{\left(e^{t \alpha}-1\right)^{2}}{1-e^{2 t \operatorname{Re} \alpha}}\right| \leq 1
$$

Since $C_{t}, D_{t}$ satisfy condition 3.5 , to get a strong continuity of $(\mathcal{T}(t))_{t \geq 0}$, we show that $\sup _{0 \leq t \leq \delta}\|\mathcal{T}(t)\|$ is finite.

$0 \leq t \leq \delta$

In the rest of the proof, we always consider $t \in[0, \delta]$, and then $\left|C_{t}\right| \leq 2,\left|B_{t}\right| \leq 1$.

For $\alpha$ satisfying conditions 3.10 , if $\operatorname{Re} \alpha=0$ and $a K+b-\bar{K}=0$, then the coefficient $A_{t}$ of $z$ in $\phi_{t}$ has modulus 1 , and so

$$
\|\mathcal{T}(t)\|=\sqrt{M\left(h_{t}, \phi_{t}\right)}=\sqrt{\left|C_{t}\right|^{2} e^{\left|B_{t}\right|^{2}}} \leq 2 e .
$$

If $\operatorname{Re} \alpha<0$, then $\left|A_{t}\right|<1$, and it follows from $(2.5)$, that

$$
\begin{aligned}
M\left(h_{t}, \phi_{t}\right) & \left.\leq 4 \exp \left(\left|B_{t}\right|^{2}+\frac{\left(\left|A_{t} B_{t}\right|+\left|D_{t}\right|\right)^{2}}{1-\left|A_{t}\right|^{2}}\right) \quad \quad \quad \quad \text { since }\left|C_{t}\right|<2\right) \\
& \left.\leq 4 \exp \left(\left|B_{t}\right|^{2}+\frac{\left(\left|B_{t}\right|+\left|D_{t}\right|\right)^{2}}{1-\left|A_{t}\right|^{2}}\right) \quad \quad \quad \text { (since }\left|A_{t}\right|<1\right) \\
& \left.\leq 4 \exp \left(1+\frac{\left(\left|B_{t}\right|+\left|D_{t}\right|\right)^{2}}{1-\left|A_{t}\right|^{2}}\right) \quad \quad \text { (since }\left|B_{t}\right|<1\right) \\
& =4 \exp \left(1+(|K|+|a K+b|)^{2} \cdot \frac{\left|e^{\alpha t}-1\right|^{2}}{1-e^{2 t \operatorname{Re} \alpha}}\right) \\
& \leq 4 e^{1+(|K|+|a K+b|)^{2}} \quad(\text { by }(3.12)) .
\end{aligned}
$$

Consequently, by 2.2 , we have

$$
\begin{aligned}
\|\mathcal{T}(t)\| & \leq \frac{\sqrt{M\left(h_{t}, \phi_{t}\right)}}{\left|A_{t}\right|} \leq \frac{\sqrt{4 e^{1+(|K|+|a K+b|)^{2}}}}{\left|e^{t \alpha}\right|} \\
& =\frac{\sqrt{4 e^{1+(|K|+|a K+b|)^{2}}}}{e^{t \operatorname{Re} \alpha}} .
\end{aligned}
$$


Since $\operatorname{Re} \alpha<0$ and $t \in[0, \delta]$, we estimate

$$
\|\mathcal{T}(t)\| \leq \frac{2 \sqrt{e^{1+(|K|+|a K+b|)^{2}}}}{e^{\delta \operatorname{Re} \alpha}}=2 e^{\frac{1}{2}\left[1+(|K|+|a K+b|)^{2}\right]-\delta \operatorname{Re} \alpha},
$$

as desired.

\subsubsection{A characterization of $\mathrm{CSO} C_{0}$-semigroups}

The following result is crucial in this section.

Theorem 3.5.5. Let $(\mathcal{T}(t))_{t \geq 0}$ be a semigroup of weighted composition operators on $\mathcal{F}^{2}(\mathbb{C})$ defined by

$$
(\mathcal{T}(t) f)(z)=h_{t}(z) f\left(\phi_{t}(z)\right), f \in \mathcal{F}^{2}(\mathbb{C}), z \in \mathbb{C},
$$

where $\left(\phi_{t}\right)_{t \geq 0}$ is a semiflow with the corresponding semicocycle $\left(h_{t}\right)_{t \geq 0}$. Then $(\mathcal{T}(t))_{t \geq 0}$ is a $\mathcal{C}_{a, b, c}$-symmetric $C_{0}$-semigroup on $\mathcal{F}^{2}(\mathbb{C})$ if and only if $\left(\phi_{t}\right)_{t \geq 0}$ and $\left(h_{t}\right)_{t \geq 0}$ satisfy either condition (3.9) or condition 3.10.

Proof. - Suppose that $(\mathcal{T}(t))_{t \geq 0}$ is a $\mathcal{C}_{a, b, c}$-symmetric $C_{0}$-semigroup. Then each $\mathcal{T}(t)$ is a $\mathcal{C}_{a, b, c}$-symmetric bounded weighted composition operator on $\mathcal{F}^{2}(\mathbb{C})$. By Proposition 3.5.3, $\left(\phi_{t}\right)$ and $\left(h_{t}\right)$ satisfy either condition 3.9 or condition 3.10 .

- Conversely, suppose that the semiflow and its semicocycle satisfy either condition (3.9) or condition 3.10). By Theorem 2.2.8, $(\mathcal{T}(t))_{t \geq 0}$ is a semigroup of $\mathcal{C}_{a, b, c^{-}}$symmetric bounded weighted composition operators on $\mathcal{F}^{2}(\mathbb{C})$, while Proposition 3.5 .4 asserts that this family must be strongly continuous.

\subsubsection{Generators of CSO $C_{0}$-semigroups}

Having discussed the structure of $\mathcal{C}_{a, b, c}$-symmetric $C_{0}$-semigroups on the Fock space $\mathcal{F}^{2}(\mathbb{C})$, the next task is to find their generators.

The lemma below is useful for obtaining explicit forms of generators. 
Lemma 3.5.6. Let $T: \operatorname{dom}(T) \rightarrow \mathbb{X}, S: \operatorname{dom}(S) \rightarrow \mathbb{X}$ be two linear operators on a Banach space $\mathbb{X}$. Suppose $T \preceq S, T$ is onto, and $S$ is one-to-one. Then $T=S$.

Proof. It suffices to prove that $\operatorname{dom}(S) \subseteq \operatorname{dom}(T)$. Indeed, let $g \in \operatorname{dom}(S)$. Putting $f=S g \in \mathbb{X}$, since $T$ is onto, there exists $h \in \operatorname{dom}(T)$ such that $f=T h$; also since $T \preceq S$, we have $T h=S h$. Thus, $S g=S h$, which implies, by the injectivity of $S$, that $g=h$, that is $g \in \operatorname{dom}(T)$.

In view of Theorem 3.5.5, we consider two cases.

- For the case when $\left(\phi_{t}\right)_{t \geq 0}$ has no fixed point, we have the following result.

Proposition 3.5.7. Let $(\mathcal{T}(t))_{t \geq 0}$ be a $\mathcal{C}_{a, b, c}$-symmetric $C_{0}$-semigroup, which is of form (3.9). Then the following statements hold:

1. Its generator $\Gamma_{1}$ is given by

$$
\Gamma_{1} f(z)=(G+a K z) f(z)+K f^{\prime}(z), f \in \operatorname{dom}\left(\Gamma_{1}\right)
$$

where

$$
\operatorname{dom}\left(\Gamma_{1}\right)=\left\{f \in \mathcal{F}^{2}(\mathbb{C}):(G+a K z) f(z)+K f^{\prime}(z) \in \mathcal{F}^{2}(\mathbb{C})\right\}
$$

2. The generator $\Gamma_{1}$ is unbounded and $\mathcal{C}_{a, b, c}$-selfadjoint.

Proof. (1) By a direct computation, for every $m \in \mathbb{N}$, we have

$$
(G+a K z) P_{m}(z)+K P_{m}^{\prime}(z)=\left\{\begin{array}{l}
G+a K z, \quad m=0 \\
m K z^{m-1}+G z^{m}+a K z^{m+1}, \quad m \geq 1,
\end{array}\right.
$$

which imply that

$$
\left\{f \in \mathcal{F}^{2}(\mathbb{C}):(G+a K z) f(z)+K f^{\prime}(z) \in \mathcal{F}^{2}(\mathbb{C})\right\} \neq \varnothing .
$$

It makes sense to consider the operator $\mathcal{Q}: \operatorname{dom}(\mathcal{Q}) \subseteq \mathcal{F}^{2}(\mathbb{C}) \rightarrow \mathcal{F}^{2}(\mathbb{C})$ given by

$$
\mathcal{Q} f(z)=(G+a K z) f(z)+K f^{\prime}(z)
$$


with

$$
\operatorname{dom}(\mathcal{Q})=\left\{f \in \mathcal{F}^{2}(\mathbb{C}):(G+a K z) f(z)+K f^{\prime}(z) \in \mathcal{F}^{2}(\mathbb{C})\right\}
$$

For an arbitrary $f \in \operatorname{dom}\left(\Gamma_{1}\right)$, by definition of a generator, we have

$$
\lim _{t \rightarrow 0^{+}}\left\|\frac{\mathcal{T}(t) f-f}{t}-\Gamma_{1} f\right\|=0,
$$

which implies, since convergence in $\mathcal{F}^{2}(\mathbb{C})$-norm implies a point convergence, that

$$
\lim _{t \rightarrow 0^{+}}\left(\frac{(\mathcal{T}(t) f)(z)-f(z)}{t}-\Gamma_{1} f(z)\right)=0, \quad \forall z \in \mathbb{C} .
$$

Consequently, taking into account of forms of $\phi_{t}$ and $h_{t}$ in 3.9 , we have

$$
\left(\Gamma_{1} f\right)(z)=\left.((\mathcal{T}(t) f)(z))^{\prime}\right|_{t=0}=(G+a K z) f(z)+K f^{\prime}(z), \quad \forall z \in \mathbb{C},
$$

which gives $\Gamma_{1} \preceq \mathcal{Q}$.

For the reverse inclusion, we make use of Lemma 3.5.6. Recall that by [4, Proposition I.5.5, Generation Theorem II.3.8]), there are constants $\omega \in \mathbb{R}, M \geq 1$ such that

$$
\|\mathcal{T}(t)\| \leq M e^{\omega t}, \quad \forall t \geq 0
$$

and moreover, $(\omega, \infty) \subseteq \rho\left(\Gamma_{1}\right)$.

Let $\ell \in(\omega, \infty)$. Then the operator $\Gamma_{1}-\ell I: \operatorname{dom}\left(\Gamma_{1}\right) \rightarrow \mathcal{F}^{2}(\mathbb{C})$ is onto. Our task is to show that $\mathcal{Q}-\ell I$ is one-to-one. Indeed, let $f \in \operatorname{ker}(\mathcal{Q}-\ell I)$, and consider the equation

$$
(\mathcal{Q}-\ell I) f(z)=(G+a K z) f(z)+K f^{\prime}(z)-\ell f(z)=0, \quad f \in \mathcal{F}^{2}(\mathbb{C})
$$

which can be rewritten as follows

$$
f^{\prime}(z)=f(z)\left(\frac{\ell-G}{K}-a z\right)
$$


Putting $g(z)=f(z) e^{\frac{G-\ell}{K} z+\frac{a z^{2}}{2}}$, we have $f(0)=g(0)$, and furthermore,

$$
\begin{aligned}
f^{\prime}(z) & =\left(g(z) e^{\frac{-G+\ell}{K} z-\frac{a z^{2}}{2}}\right)^{\prime} \\
& =g^{\prime}(z) e^{\frac{\ell-G}{K} z-\frac{a z^{2}}{2}}+g(z)\left(e^{\frac{\ell-G}{K} z-\frac{a z^{2}}{2}}\right)^{\prime} \\
& =g^{\prime}(z) e^{\frac{\ell-G}{K} z-\frac{a z^{2}}{2}}+f(z)\left(\frac{\ell-G}{K}-a z\right) .
\end{aligned}
$$

Hence, equation (3.14) is reduced to

$$
g^{\prime}(z) e^{\frac{\ell-G}{K} z-\frac{a z^{2}}{2}}=0 \Longleftrightarrow g(z)=g(0), \quad \forall z \in \mathbb{C} .
$$

So, equation (3.14) has the general solution

$$
f(z)=f(0) e^{\frac{\ell-G}{K} z-\frac{a z^{2}}{2}} .
$$

Note that $|a|=1$ by $(2.10$. Thus, by [13, Theorem 1.1],

$$
f \in \mathcal{F}^{2}(\mathbb{C}) \Longleftrightarrow f(0)=0
$$

Thus, $f=\mathbf{0}$, and $\mathcal{Q}-\ell I$ is one-to-one. We can conclude, by Lemma 3.5.6, that $\Gamma_{1}=\mathcal{Q}$.

(2) By Theorem 3.2.4, $\Gamma_{1}$ is $\mathcal{C}_{a, b, c^{-}}$selfadjoint, while its unboundedness is obtained by estimating the norm of (3.13). Indeed, for every $m \geq 2$, we have

$$
\begin{aligned}
\Gamma_{1} P_{m}(z) & =(G+a K z) z^{m}+K m z^{m-1} \\
& =G P_{m}(z)+a K P_{m+1}(z)+m K P_{m-1}(z)
\end{aligned}
$$

which implies, since $\left(P_{m}\right)$ is an orthogonal basis, that

$$
\begin{aligned}
\left\|\Gamma_{1} P_{m}\right\|^{2} & =|G|^{2}\left\|P_{m}\right\|^{2}+|a K|^{2}\left\|P_{m+1}\right\|^{2}+|m K|^{2}\left\|P_{m-1}\right\|^{2} \\
& =\left\|P_{m}\right\|^{2}\left[\left(1+|a|^{2}\right)|K|^{2} m+|G|^{2}+|a|^{2}|K|^{2}\right] .
\end{aligned}
$$

Note that the last equality holds because

$$
\left\|P_{m+1}\right\|^{2}=(m+1)\left\|P_{m}\right\|^{2}, \quad m^{2}\left\|P_{m-1}\right\|^{2}=m\left\|P_{m}\right\|^{2} .
$$


Setting $Q_{m}=\frac{P_{m}}{\left\|P_{m}\right\|}$, we have

$$
\left\|\Gamma_{1} Q_{m}\right\|^{2}=\left(1+|a|^{2}\right)|K|^{2} m+|G|^{2}+|a|^{2}|K|^{2} \geq|K|^{2} m .
$$

Clearly, the right-hand side of the above inequality converges to $\infty$ as $m \rightarrow \infty$. Thus, $\Gamma_{1}$ is unbounded.

- For the case when $\left(\phi_{t}\right)_{t \geq 0}$ has one fixed point, the generator of the $\mathcal{C}_{a, b, c^{-}}$ symmetric $C_{0}$-semigroup $(\mathcal{T}(t))_{t \geq 0}$ is given by the following proposition.

Proposition 3.5.8. Let $(\mathcal{T}(t))_{t \geq 0}$ be a $\mathcal{C}_{a, b, c}$-symmetric $C_{0}$-semigroup, which is of form (3.10). Then the following statements hold:

1. Its generator $\Gamma_{2}$ is given by

$$
\Gamma_{2} f(z)=(G-\alpha(a K+b) z) f(z)+\alpha(z-K) f^{\prime}(z)
$$

where

$$
\operatorname{dom}\left(\Gamma_{2}\right)=\left\{f \in \mathcal{F}^{2}(\mathbb{C}):(G-\alpha(a K+b) z) f(z)+\alpha(z-K) f^{\prime}(z) \in \mathcal{F}^{2}(\mathbb{C})\right\}
$$

2. The generator $\Gamma_{2}$ is unbounded and $\mathcal{C}_{a, b, c}$-selfadjoint.

Proof. It is similar to Proposition 3.5.7.

(1) By a direct computation, for every $m \in \mathbb{N}$, we have

$$
\begin{aligned}
& (G-\alpha(a K+b) z) P_{m}(z)+\alpha(z-K) P_{m}^{\prime}(z) \\
& =\left\{\begin{array}{l}
G-\alpha(a K+b) z, \quad m=0, \\
-m K \alpha z^{m-1}+(G+m \alpha) z^{m}-\alpha(a K+b) z^{m+1}, \quad m \geq 1,
\end{array}\right.
\end{aligned}
$$

which imply that

$$
\left\{f \in \mathcal{F}^{2}(\mathbb{C}):(G-\alpha(a K+b) z) f(z)+\alpha(z-K) f^{\prime}(z) \in \mathcal{F}^{2}(\mathbb{C})\right\} \neq \varnothing
$$


It makes sense to consider the operator $\mathcal{Q}: \operatorname{dom}(\mathcal{Q}) \subseteq \mathcal{F}^{2}(\mathbb{C}) \rightarrow \mathcal{F}^{2}(\mathbb{C})$ given by

$$
\mathcal{Q} f(z)=(G-\alpha(a K+b) z) f(z)+\alpha(z-K) f^{\prime}(z)
$$

with

$\operatorname{dom}(\mathcal{Q})=\left\{f \in \mathcal{F}^{2}(\mathbb{C}):(G-\alpha(a K+b) z) f(z)+\alpha(z-K) f^{\prime}(z) \in \mathcal{F}^{2}(\mathbb{C})\right\}$

For an arbitrary element $f \in \operatorname{dom}\left(\Gamma_{2}\right)$, by definition of a generator, we have

$$
\lim _{t \rightarrow 0^{+}}\left\|\frac{\mathcal{T}(t) f-f}{t}-\Gamma_{2} f\right\|=0,
$$

which implies, since convergence in the $\mathcal{F}^{2}(\mathbb{C})$-norm implies a point convergence, that

$$
\lim _{t \rightarrow 0^{+}}\left(\frac{(\mathcal{T}(t) f)(z)-f(z)}{t}-\Gamma_{2} f(z)\right)=0, \quad \forall z \in \mathbb{C} .
$$

Consequently, taking into account of forms of $\phi_{t}$ and $h_{t}$ in 3.10, we have

$$
\left(\Gamma_{2} f\right)(z)=\left.((\mathcal{T}(t) f)(z))^{\prime}\right|_{t=0}=(G-\alpha(a K+b) z) f(z)+\alpha(z-K) f^{\prime}(z), z \in \mathbb{C},
$$

which gives $\Gamma_{2} \preceq \mathcal{Q}$.

For the reverse inclusion, we make use of Lemma 3.5.6. Recall that by [4, Proposition I.5.5, Generation Theorem II.3.8]), there are constants $\omega \in \mathbb{R}, M \geq 1$, such that

$$
\|\mathcal{T}(t)\| \leq M e^{\omega t}, \quad \forall t \geq 0
$$

and moreover, $(\omega, \infty) \subseteq \rho\left(\Gamma_{2}\right)$.

Let $\ell \in(\omega, \infty)$. Then the operator $\Gamma_{2}-\ell I: \operatorname{dom}\left(\Gamma_{2}\right) \rightarrow \mathcal{F}^{2}(\mathbb{C})$ is onto. Our task is to show that $\mathcal{Q}-\ell I$ is one-to-one. Indeed, let $f \in \operatorname{ker}(\mathcal{Q}-\ell I)$, and consider the equation

$$
(\mathcal{Q}-\ell I) f(z)=(G-\ell-\alpha(a K+b) z) f(z)+\alpha(z-K) f^{\prime}(z)=0, \quad f \in \mathcal{F}^{2}(\mathbb{C}) .
$$


Letting $z=\phi_{t}(w)$, we get

$$
\left(G-\ell-\alpha(a K+b) \phi_{t}(w)\right) f\left(\phi_{t}(w)\right)+\alpha(z-K) f^{\prime}\left(\phi_{t}(w)\right)=0
$$

Since

$$
\frac{\partial \phi_{t}(w)}{\partial t}=\left(e^{t \alpha} w+K\left(1-e^{t \alpha}\right)\right)^{\prime}=\alpha e^{t \alpha}(w-K)=\alpha(z-K),
$$

the above equation is rewritten as follows

$$
\left(G-\ell-\alpha(a K+b) \phi_{t}(w)\right) f\left(\phi_{t}(w)\right)+\frac{\partial \phi_{t}(w)}{\partial t} f^{\prime}\left(\phi_{t}(w)\right)=0,
$$

which is equivalent to

$$
\left(G-\ell-\alpha(a K+b) \phi_{t}(w)\right) v(t)+v^{\prime}(t)=0
$$

where $v(t)=f\left(\phi_{t}(w)\right)$.

Setting

$$
u(t)=v(t) e^{-(a K+b) K\left(1+\alpha t-e^{\alpha t}\right)-t(\ell-G)-\left(e^{\alpha t}-1\right)(a K+b) w},
$$

by the product rule for derivatives, we have

$$
\begin{aligned}
v^{\prime}(t)= & \left(u(t) e^{(a K+b) K\left(1+\alpha t-e^{\alpha t}\right)+t(\ell-G)+\left(e^{\alpha t}-1\right)(a K+b) w}\right)^{\prime} \\
= & u^{\prime}(t) e^{(a K+b) K\left(1+\alpha t-e^{\alpha t}\right)+t(\ell-G)+\left(e^{\alpha t}-1\right)(a K+b) w} \\
& +\left[(a K+b) \alpha K\left(1-e^{\alpha t}\right)+\ell-G+\alpha e^{\alpha t}(a K+b) w\right] v(t) \\
= & u^{\prime}(t) e^{(a K+b) K\left(1+\alpha t-e^{\alpha t}\right)+t(\ell-G)+\left(e^{\alpha t}-1\right)(a K+b) w} \\
& +\left(-G+\ell+\alpha(a K+b) \phi_{t}(w)\right) v(t)
\end{aligned}
$$

which implies, by 3.16$)$, that $u^{\prime}(t)=0$. Hence,

$$
u(t)=u(0), \quad \forall t \geq 0
$$

Taking into account of the form of $u(t)$, we have

$$
f\left(\phi_{t}(w)\right)=e^{(a K+b) K\left(1+\alpha t-e^{\alpha t}\right)+t(\ell-G)+\left(e^{\alpha t}-1\right)(a K+b) w} f(w), \quad \forall w \in \mathbb{C},
$$


and so,

$$
\begin{aligned}
\mathcal{T}(t) f(w) & =h_{t}(w) f\left(\phi_{t}(w)\right)=h_{t}(w) e^{(a K+b) K\left(1+\alpha t-e^{\alpha t}\right)+t(\ell-G)+\left(e^{\alpha t}-1\right)(a K+b) w} f(w) \\
& =e^{\ell t} f(w)
\end{aligned}
$$

Thus,

$$
e^{t \ell}\|f\|=\|\mathcal{T}(t) f\| \leq M e^{\omega t}\|f\|, \quad \forall t \geq 0 .
$$

Since $\ell>\omega, f$ must be $\mathbf{0}$. Thus, $\mathcal{Q}-\ell I$ is one-to-one. By Lemma 3.5.6, we conclude that $\Gamma_{2}=\mathcal{Q}$.

(2) By Theorem 3.2.4, $\Gamma_{2}$ is $\mathcal{C}_{a, b, c}$-selfadjoint, while its unboundedness is obtained by estimating the norm of (3.15). Indeed, for every $m \geq 2$, we have

$$
\Gamma_{2} P_{m}=-m K \alpha P_{m-1}+(G+m \alpha) P_{m}-\alpha(a K+b) P_{m+1},
$$

which implies, since $\left(P_{m}\right)$ is an orthogonal basis, that

$$
\begin{aligned}
\left\|\Gamma_{2} P_{m}\right\|^{2} & =|\alpha K m|^{2}\left\|P_{m-1}\right\|^{2}+|G+\alpha m|^{2}\left\|P_{m}\right\|^{2}+|\alpha(a K+b)|^{2}\left\|P_{m+1}\right\|^{2} \\
& =\left\|P_{m}\right\|^{2}\left[|\alpha K|^{2} m+|G+\alpha m|^{2}+|\alpha(a K+b)|^{2}(m+1)\right] .
\end{aligned}
$$

Setting $f_{m}=\frac{P_{m}}{\left\|P_{m}\right\|}$, we obtain

$$
\begin{aligned}
\left\|\Gamma_{2} f_{m}\right\|^{2} & =|\alpha K|^{2} m+|G+\alpha m|^{2}+|\alpha(a K+b)|^{2}(m+1) \\
& \geq|G+\alpha m|^{2}
\end{aligned}
$$

Clearly, the right-hand side of the above inequality converges to $\infty$ as $m \rightarrow \infty$. Thus, $\Gamma_{2}$ is unbounded.

\subsection{Unitary $C_{0}$-semigroups on $\mathcal{F}^{2}(\mathbb{C})$}

Theorem 3.3.3 shows that every unitary group is complex symmetric. A natural question to ask is whether $\mathcal{C}_{a, b, c}$-symmetric $C_{0}$-semigroups obtained in Theorem 3.5 .5 are unitary. In this section, we characterize the structure of the family 
$(\mathcal{T}(t))_{t \geq 0}$, when it is a unitary $C_{0}$-semigroup on $\mathcal{F}^{2}(\mathbb{C})$. This allows us to construct a $\mathcal{C}_{a, b, c}$-symmetric $C_{0}$-semigroup, which is not unitary. Hence, the concept of complex symmetric $C_{0}$-semigroups is really a generalization.

\subsubsection{A characterization of unitary $C_{0}$-semigroups}

In the result below, we give the structure of the family $(\mathcal{T}(t))_{t \geq 0}$ when it is a semigroup of unitary weighted composition operators on the Fock space $\mathcal{F}^{2}(\mathbb{C})$.

Proposition 3.6.1. Let $(\mathcal{T}(t))_{t \geq 0}$ be a semigroup of weighted composition operators induced by the semiflow $\left(\phi_{t}\right)_{t \geq 0}$ and the corresponding semicocycle $\left(h_{t}\right)_{t \geq 0}$. Suppose that $\mathcal{T}(t)$ is unitary on $\mathcal{F}^{2}(\mathbb{C})$, for every $t \geq 0$. Then

1. If $\left(\phi_{t}\right)_{t \geq 0}$ has no fixed point, then there exist complex constants $K \neq 0$ and $G$ with $\operatorname{Re} G=0$, such that

$$
\left\{\begin{array}{l}
\phi_{t}(z)=z+K t \\
h_{t}(z)=e^{t G-z t \bar{K}-t^{2}|K|^{2} / 2} .
\end{array}\right.
$$

2. If $\left(\phi_{t}\right)_{t \geq 0}$ has one fixed point, then there exist constants $d \in \mathbb{R} \backslash\{0\}, \beta \in \mathbb{R}$, and $K \in \mathbb{C}$, such that

$$
\left\{\begin{array}{l}
\phi_{t}(z)=z A_{t}+B_{t} \\
h_{t}(z)=E_{t} e^{-z A_{t} \overline{B_{t}}-\left|B_{t}\right|^{2} / 2}
\end{array}\right.
$$

where $A_{t}=e^{i t d}, B_{t}=K\left(1-A_{t}\right), E_{t}=e^{i\left(\beta t+|K|^{2}(\sin t d-t d)\right)}$.

Proof. Since $\mathcal{T}(t)$ is unitary on $\mathcal{F}^{2}(\mathbb{C})$, by Proposition 1.4 .7 ,

$$
\phi_{t}(z)=A_{t} z+B_{t}, \quad h_{t}(z)=E_{t} e^{-z A_{t} \overline{B_{t}}-\left|B_{t}\right|^{2} / 2},
$$

where $A_{t}, B_{t}, E_{t}$ are functions of one variable $t$ with $\left|A_{t}\right|=\left|E_{t}\right|=1$. 
We have

$$
\begin{aligned}
h_{t}(z) h_{s}\left(\phi_{t}(z)\right) & =E_{t} e^{-z A_{t} \overline{B_{t}}-\left|B_{t}\right|^{2} / 2} E_{s} e^{-\left(A_{t} z+B_{t}\right) A_{s} \overline{B_{s}}-\left|B_{s}\right|^{2} / 2} \\
& =E_{t} E_{s} e^{-z A_{t}\left(\overline{B_{t}}+A_{s} \overline{B_{s}}\right)-A_{s} B_{t} \overline{B_{s}}-\frac{\left|B_{t}\right|^{2}+\left|B_{s}\right|^{2}}{2}}
\end{aligned}
$$

which implies, as $h_{t+s}=h_{t} \cdot h_{s} \circ \phi_{t}$, that

$E_{t+s} e^{-z A_{t+s} \overline{B_{t+s}}-\left|B_{t+s}\right|^{2} / 2}=E_{t} E_{s} e^{-z A_{t}\left(\overline{B_{t}}+A_{s} \overline{B_{s}}\right)-A_{s} B_{t} \overline{B_{s}}-\frac{\left|B_{t}\right|^{2}+\left|B_{s}\right|^{2}}{2}}, \quad \forall z \in \mathbb{C}, \forall t, s \geq 0$.

In particular, for $z=0$, we get

$$
E_{t+s}=E_{t} E_{s} e^{\frac{\left|B_{t+s}\right|^{2}-\left|B_{t}\right|^{2}-\left|B_{s}\right|^{2}-2 A_{s} B_{t} \overline{B_{s}}}{2}}, \quad \forall t, s \geq 0
$$

1) Suppose that $\left(\phi_{t}\right)_{t \geq 0}$ has no fixed point. By Proposition 3.4.2(1),

$$
A_{t} \equiv 1, \quad B_{t}=t K
$$

and so,

$$
\left|B_{t+s}\right|^{2}-\left|B_{t}\right|^{2}-\left|B_{s}\right|^{2}-2 A_{s} B_{t} \overline{B_{s}}=(t+s)^{2}|K|^{2}-t^{2}|K|^{2}-s^{2}|K|^{2}-2 t K \overline{s K}=0 .
$$

Now we apply Lemma 3.5.1 to 3.19 to get $E_{t}=e^{t G}$ with some constant $G$. Since $\left|E_{t}\right|=1$, we must have $\operatorname{Re} G=0$. Then $h_{t}(z)=e^{t G-t^{2}|K|^{2} / 2-z t \bar{K}}$, with $\operatorname{Re} G=0$.

2) For the case when $\left(\phi_{t}\right)_{t \geq 0}$ has one fixed point, it is similar. By Proposition $3.4 .2(2)$

$$
A_{t}=e^{t \alpha}, \quad B_{t}=K\left(1-e^{t \alpha}\right)=K\left(1-A_{t}\right)
$$

Furthermore, since $\left|A_{t}\right|=1, \alpha=i d$, for some $d \in \mathbb{R} \backslash\{0\}$. We compute some following quantities:

$$
\begin{aligned}
\left|B_{t}\right|^{2} & =|K|^{2} \cdot\left|1-A_{t}\right|^{2}=\left(1+\left|A_{t}\right|^{2}-A_{t}-\overline{A_{t}}\right)|K|^{2} \\
& =\left(2-A_{t}-\overline{A_{t}}\right)|K|^{2} \quad\left(\text { since }\left|A_{t}\right|=1\right),
\end{aligned}
$$


and

$$
\begin{aligned}
A_{s} B_{t} \overline{B_{s}} & =|K|^{2} A_{s}\left(1-A_{t}\right)\left(1-\overline{A_{s}}\right)=|K|^{2}\left(1-A_{t}\right)\left(A_{s}-\left|A_{s}\right|^{2}\right) \\
& =|K|^{2}\left(1-A_{t}\right)\left(A_{s}-1\right)=|K|^{2}\left(A_{s}-1-A_{t+s}+A_{t}\right) .
\end{aligned}
$$

From these,

$$
\begin{aligned}
& \left|B_{t+s}\right|^{2}-\left|B_{t}\right|^{2}-\left|B_{s}\right|^{2}-2 A_{s} B_{t} \overline{B_{s}} \\
& =|K|^{2}\left(2-A_{t+s}-\overline{A_{t+s}}-2+A_{t}+\overline{A_{t}}-2+A_{s}+\overline{A_{s}}-2 A_{s}+2+2 A_{t+s}-2 A_{t}\right) \\
& =2 i|K|^{2}\left(\operatorname{Im} A_{t+s}-\operatorname{Im} A_{t}-\operatorname{Im} A_{s}\right) \\
& =2 i|K|^{2}(\sin (t+s) d-\sin t d-\sin s d) .
\end{aligned}
$$

Then equation (3.19) is reduced to

$$
E_{t+s}=E_{t} E_{s} e^{i|K|^{2}(\sin (t+s) d-\sin t d-\sin s d)}, \quad E_{0}=1 .
$$

Now by Lemma 3.5.1(4), we get

$$
E_{t}=e^{t G+i|K|^{2}(\sin t d-t d)},
$$

which implies that $\left|E_{t}\right|=e^{t \operatorname{Re} G}$. Since $\left|E_{t}\right|=1, G=i \beta$, for some $\beta \in \mathbb{R}$.

The proof is complete.

Theorem 3.6.2. Let $(\mathcal{T}(t))_{t \geq 0}$ be a semigroup of weighted composition operators on $\mathcal{F}^{2}(\mathbb{C})$ defined by

$$
(\mathcal{T}(t) f)(z)=h_{t}(z) f\left(\phi_{t}(z)\right), f \in \mathcal{F}^{2}(\mathbb{C}), z \in \mathbb{C},
$$

where $\left(\phi_{t}\right)_{t \geq 0}$ is a semiflow with the corresponding semicocycle $\left(h_{t}\right)_{t \geq 0}$. This semigroup is a $C_{0}$-semigroup of unitary operators if and only if $\left(\phi_{t}\right)$ and $\left(h_{t}\right)$ satisfy either conditions 3.17) or conditions 3.18). 
Proof. • Suppose that $(\mathcal{T}(t))_{t \geq 0}$ is a $C_{0}$-semigroup of unitary operators. By Proposition 3.6.1, we obtain 3.17)-3.18).

- Conversely, suppose that $\left(\phi_{t}\right)$ and $\left(h_{t}\right)$ satisfy either (3.17) or 3.18). In these cases, conditions 3.5 hold. On the other hand, by Proposition 1.4.7, $\mathcal{T}(t)$ is unitary for every $t \geq 0$. It is well known that the norm of unitary operators is always 1 . Thus, $\|\mathcal{T}(t)\|=1$ for every $t \geq 0$. Hence, by Proposition 3.5.2, we get the strong continuity of $(\mathcal{T}(t))_{t \geq 0}$.

\subsubsection{Generators of unitary $C_{0}$-semigroups}

In the preceding subsection, $C_{0}$-semigroups of unitary weighted composition operators are constructed on the Fock space $\mathcal{F}^{2}(\mathbb{C})$. The proposition below shows that these semigroups are contained in the family of $\mathcal{C}_{a, b, c}$-symmetric $C_{0}$-semigroups as a very particular case.

Proposition 3.6.3. Suppose that $(\mathcal{T}(t))_{t \geq 0}$ is a $C_{0}$-semigroup of unitary weighted composition operators on $\mathcal{F}^{2}(\mathbb{C})$. The following statements hold:

1. If $\left(\phi_{t}\right)_{t \geq 0}$ has no fixed point, that is conditions 3.17 hold, then $(\mathcal{T}(t))_{t \geq 0}$ is a $\mathcal{C}_{a, b, c}$-symmetric $C_{0}$-semigroup, with

$$
a=-\frac{\bar{K}}{K}, \quad b=0, \quad c=1 .
$$

2. If $\left(\phi_{t}\right)_{t \geq 0}$ has one fixed point, that is conditions (3.18) hold, then $(\mathcal{T}(t))_{t \geq 0}$ is a $\mathcal{C}_{a, b, c}$-symmetric $C_{0}$-semigroup, with

$$
a=\left\{\begin{array}{ll}
\bar{K} / K & (K \neq 0) \\
1 & (K=0),
\end{array} \quad b=0, \quad c=1 .\right.
$$

Here $K$ is as in Proposition 3.6.1. 
Proof. To show that $(\mathcal{T}(t))_{t \geq 0}$ is a $\mathcal{C}_{a, b, c}$-symmetric $C_{0}$-semigroup, we make use of Theorem 3.5.5. Note that by Theorem 3.6.2. $(\mathcal{T}(t))_{t \geq 0}$ is either of form 3.17) or form (3.18). Our task is to show that 3.17) and 3.18) are equivalent respectively to 3.9 and 3.10 .

(1) To prove that 3.17$)$ (3.9), we suppose that $\left(\phi_{t}\right)_{t \geq 0}$ has no fixed point. By Proposition 3.6.1, we have

$$
\phi_{t}(z)=z+K t, h_{t}(z)=e^{t G-t^{2}|K|^{2} / 2-z t \bar{K}}
$$

for some $K \in \mathbb{C} \backslash\{0\}$ and $G \in \mathbb{C}$ with $\operatorname{Re} G=0$.

For choosing $3.20, \mathcal{C}_{a, b, c}$ is a weighted composition conjugation by Theorem 2.1.18. Choosing $a=-\frac{\bar{K}}{K}$ gives $\bar{K}=-a K$ and $|K|^{2}=-a K^{2}$. So, we can rewrite

$$
h_{t}(z)=e^{t G+a K^{2} t^{2} / 2-z t \bar{K}}=e^{t G+a K^{2} t^{2} / 2+a K t z} .
$$

The above equality shows $(\mathcal{T}(t))_{t \geq 0}$ is of form $(3.9)$ and hence, it is $\mathcal{C}_{a, b, c}$-symmetric.

2 ) To show that $(3.18 \Longleftrightarrow 3.10)$, we suppose that $\left(\phi_{t}\right)_{t \geq 0}$ has one fixed point. Note that for choosing $(3.21), \mathcal{C}_{a, b, c}$ is a weighted composition conjugation by Theorem 2.1.18, By Proposition 3.6.1, there exist constants $d \in \mathbb{R} \backslash\{0\}, \beta \in \mathbb{R}$, and $K \in \mathbb{C}$, such that

$$
\phi_{t}(z)=z A_{t}+B_{t}, \quad h_{t}(z)=E_{t} e^{-z A_{t} \overline{B_{t}}-\left|B_{t}\right|^{2} / 2},
$$

where $A_{t}=e^{i t d}, B_{t}=K\left(1-A_{t}\right)$, and $E_{t}=e^{i\left(\beta t+|K|^{2}(\sin t d-t d)\right)}$.

- If $K=0$, then $B_{t}=0, E_{t}=e^{i \beta t}$, and so,

$$
\phi_{t}(z)=z e^{i t d} \text { and } h_{t}(z)=e^{i \beta t}, z \in \mathbb{C} .
$$

This shows that $(\mathcal{T}(t))_{t \geq 0}$ has form 3.10 with $a=1, b=0, c=1, \alpha=i d, G=i \beta$, and therefore, it is $\mathcal{C}_{a, b, c}$-symmetric by Theorem 3.5.5.

- If $K \neq 0$, then for choosing $a=\bar{K} / K, b=0, c=1$, we have

$$
\bar{K}=a K+b, \quad|K|^{2}=K(a K+b) .
$$


In this case,

$$
\begin{aligned}
& -A_{t} \overline{B_{t}}=-e^{i t d} \bar{K}\left(1-e^{-i t d}\right)=\bar{K}\left(1-e^{i t d}\right)=(a K+b)\left(1-e^{i t d}\right), \\
& i\left(\beta t+|K|^{2}(\sin t d-t d)\right)=i \beta t+K(a K+b)(i \sin t d-i t d), \\
& \frac{\left|B_{t}\right|^{2}}{2}=\frac{|K|^{2}\left|1-e^{i t d}\right|^{2}}{2}=|K|^{2}(1-\cos t d)=K(a K+b)(1-\cos t d),
\end{aligned}
$$

and hence,

$$
\begin{aligned}
h_{t}(z)=E_{t} e^{-z A_{t} \overline{B_{t}}-\left|B_{t}\right|^{2} / 2} & =e^{i \beta t+K(a K+b)(i \sin t d-i t d-1+\cos t d)+(a K+b)\left(1-e^{i t d}\right) z} \\
& =e^{i \beta t+K(a K+b)\left(e^{i t d}-i t d-1\right)+(a K+b)\left(1-e^{i t d}\right) z}
\end{aligned}
$$

while

$$
\phi_{t}(z)=z e^{i t d}+K\left(1-e^{i t d}\right) .
$$

This shows that $(\mathcal{T}(t))_{t \geq 0}$ has form 3.10 of Proposition 3.5.3 with again $\alpha=$ $i d, G=i \beta$, and hence, it is $\mathcal{C}_{a, b, c}$-symmetric by Theorem 3.5 .5 .

Propositions 3.5.7 3.5.8 are reduced, for unitary $C_{0}$-semigroups, to the following results.

Proposition 3.6.4. If $(\mathcal{T}(t))_{t \geq 0}$ is of form (3.17), then

1. its generator $\Gamma_{1}$ is given by

$$
\begin{gathered}
\left(\Gamma_{1} f\right)(z)=(G-z \bar{K}) f(z)+K f^{\prime}(z), z \in \mathbb{C} \\
\operatorname{dom}\left(\Gamma_{1}\right)=\left\{f \in \mathcal{F}^{2}(\mathbb{C}):(G-z \bar{K}) f(z)+K f^{\prime}(z) \in \mathcal{F}^{2}(\mathbb{C})\right\} .
\end{gathered}
$$

2. the generator $\Gamma_{1}$ is unbounded and skew-selfadjoint.

Proposition 3.6.5. If $(\mathcal{T}(t))_{t \geq 0}$ is of form 3.18 , then

1. its generator $\Gamma_{2}$ is given by

$$
\begin{gathered}
\left(\Gamma_{2} f\right)(z)=i(\beta-z \bar{K} d) f(z)+i d(z-K) f^{\prime}(z), z \in \mathbb{C}, \\
\operatorname{dom}\left(\Gamma_{2}\right)=\left\{f \in \mathcal{F}^{2}(\mathbb{C}): i(\beta-z \bar{K} d) f(z)+i d(z-K) f^{\prime}(z) \in \mathcal{F}^{2}(\mathbb{C})\right\} .
\end{gathered}
$$

2. the generator $\Gamma_{2}$ is unbounded and skew-selfadjoint. 



\section{Chapter 4}

\section{Complex symmetric bounded}

\section{weighted composition operators}

on $\mathcal{F}^{2}\left(\mathbb{C}^{n}\right)$

In this chapter, we study a complex symmetry in the Fock spaces of $\mathbb{C}^{n}$ with $n \geq 2$. As in the case of one variable, we also characterize anti-linear weighted composition operators, which are conjugations on $\mathcal{F}^{2}\left(\mathbb{C}^{n}\right)$. In comparison with Chapter 1 , our approach is completely different. It is inspired by the following simple note: an anti-linear mapping is a conjugation if and only if it is both unitary and selfadjoint. To get weighted composition conjugations on $\mathcal{F}^{2}\left(\mathbb{C}^{n}\right)$, we characterize all selfadjoint as well as all unitary anti-linear weighted composition operators. In our opinion, this method is more useful for studying a complex symmetry in other function spaces, including Hardy spaces, on which unitariness was studied successfully (see [17]).

Finally, we investigate which bounded weighted composition operators can be complex symmetric with weighted composition conjugations on $\mathcal{F}^{2}\left(\mathbb{C}^{n}\right)$.

In this chapter, the symbol $\kappa \eta$ is used to indicate the composition $\kappa \circ \eta$, where 
$\eta$ is a mapping from $\mathbb{C}^{n}$ into itself and $\kappa$ is the standard conjugation on $\mathbb{C}^{n}$ defined by $(1.3)$, that is

$$
\kappa\left(z_{1}, z_{2}, \ldots, z_{n}\right)=\left(\overline{z_{1}}, \overline{z_{2}}, \ldots, \overline{z_{n}}\right)
$$

\subsection{Anti-linear weighted composition operators}

We start the section with a direct generalization of Lemma 2.1.2.

Lemma 4.1.1. The operator $\mathcal{K} f(z)=\overline{f(\kappa(z))}$ is a conjugation on $\mathcal{F}^{2}\left(\mathbb{C}^{n}\right)$.

Proof. For every $f(z)=\sum_{\tau} f_{\tau} z^{\tau} \in \mathcal{F}^{2}\left(\mathbb{C}^{n}\right)$ (here $\tau$ is a multi-index), we have

$$
(\mathcal{K} f)(z)=\overline{f(\bar{z})}=\overline{\sum_{\tau} f_{\tau} \bar{z}^{\tau}}=\sum_{\tau} \overline{f_{\tau}} z^{\tau}
$$

which implies, as $\left(z^{\tau}\right)$ is an orthogonal basis in $\mathcal{F}^{2}\left(\mathbb{C}^{n}\right)$, that

$$
\|\mathcal{K} f\|^{2}=\sum_{\tau}\left|\overline{f_{\tau}}\right|^{2}\left\|z^{\tau}\right\|^{2}=\|f\|^{2}
$$

The last equality shows that $\mathcal{K} f \in \mathcal{F}^{2}\left(\mathbb{C}^{n}\right)$, and moreover, $\mathcal{K}$ is isometric.

In addition, the operator $\mathcal{K}$ is involutive, since

$$
\mathcal{K}^{2} f(z)=\mathcal{K}\left(\sum_{\tau} \overline{f_{\tau}} z^{\tau}\right)(z)=\sum_{\tau} f_{\tau} z^{\tau}, \quad \forall z \in \mathbb{C}^{n}
$$

As for the case of one dimension, the operator $\mathcal{K}$ is a particular case of the so-called anti-linear weighted composition operators. For $\xi \in \operatorname{Hol}\left(\mathbb{C}^{n}\right)$ and $\eta \in$ $\operatorname{HSel}\left(\mathbb{C}^{n}\right)$, this kind operator, denoted as $\mathcal{A}_{\xi, \eta}$, is defined by

$$
\left(\mathcal{A}_{\xi, \eta} f\right)(z)=\xi(z) \overline{f(\kappa \eta(z))}
$$

The following observation is a direct generalization of Proposition 2.1.1. 
Proposition 4.1.2. The operator $\mathcal{A}_{\xi, \eta}$ is anti-linear.

Proof. For every $f, g \in \mathcal{F}^{2}\left(\mathbb{C}^{n}\right)$, and $\alpha, \beta \in \mathbb{C}$, we have

$$
\begin{aligned}
\mathcal{A}_{\xi, \eta}(\alpha f+\beta g)(z) & =\xi(z) \overline{(\alpha f+\beta g)(\overline{\eta(z)})}=\xi(z) \overline{(\alpha f)(\overline{\eta(z)})}+\xi(z) \overline{(\beta g)(\overline{\eta(z)})} \\
& =\xi(z) \bar{\alpha} \overline{f(\overline{\eta(z)})}+\xi(z) \bar{\beta} \overline{g(\overline{\eta(z)})}=\bar{\alpha}\left(\mathcal{A}_{\xi, \eta} f\right)(z)+\bar{\beta}\left(\mathcal{A}_{\xi, \eta} g\right)(z) .
\end{aligned}
$$

As for the case of one variable, there is a relationship between $W_{\xi, \eta}$ and $\mathcal{A}_{\xi, \eta}$, in which the conjugation $\mathcal{K}$ plays an essential role.

Proposition 4.1.3. We have $\mathcal{A}_{\xi, \eta}=W_{\xi, \eta} \mathcal{K}$, or the same $W_{\xi, \eta}=\mathcal{A}_{\xi, \eta} \mathcal{K}$.

Proof. Take arbitrarily $f(z) \in \mathcal{F}^{2}\left(\mathbb{C}^{n}\right)$. Since $\mathcal{K} f(z)=\overline{f(\kappa(z))}$, for all $z \in \mathbb{C}^{n}$, we have

$$
\left(W_{\xi, \eta} \mathcal{K}\right) f(z)=\xi(z) \mathcal{K} f(\eta(z))=\xi(z) \overline{f(\kappa \eta(z))}=\left(\mathcal{A}_{\xi, \eta} f\right)(z)
$$

Furthermore, the formula $\mathcal{A}_{\xi, \eta}=W_{\xi, \eta} \mathcal{K}$ is equivalent to $W_{\xi, \eta}=\mathcal{A}_{\xi, \eta} \mathcal{K}$, because the operator $\mathcal{K}$ is involutive.

Proposition 4.1 .3 allows us to show that $W_{\xi, \eta}$ and $\mathcal{A}_{\xi, \eta}$ have several common important properties.

Theorem 4.1.4. Let $\xi \in \operatorname{Hol}\left(\mathbb{C}^{n}\right)$ and $\eta \in \operatorname{HSel}\left(\mathbb{C}^{n}\right)$. The following assertions hold:

1. $\mathcal{A}_{\xi, \eta}$ is bounded on $\mathcal{F}^{2}\left(\mathbb{C}^{n}\right)$ if and only if so is $W_{\xi, \eta}$. In this case, $\left\|W_{\xi, \eta}\right\|=$ $\left\|\mathcal{A}_{\xi, \eta}\right\|$.

2. $\mathcal{A}_{\xi, \eta}$ is isometric on $\mathcal{F}^{2}\left(\mathbb{C}^{n}\right)$ if and only if so is $W_{\xi, \eta}$.

3. $\mathcal{A}_{\xi, \eta}$ is invertible on $\mathcal{F}^{2}\left(\mathbb{C}^{n}\right)$ if and only if so is $W_{\xi, \eta}$.

4. $\mathcal{A}_{\xi, \eta}$ is unitary on $\mathcal{F}^{2}\left(\mathbb{C}^{n}\right)$ if and only if so is $W_{\xi, \eta}$. 
Proof. The proofs for (1)-(2) are similar to Theorem 2.1.4.

(3) Suppose that $\mathcal{A}_{\xi, \eta}$ is bounded invertible. Then $\mathcal{A}_{\xi, \eta}^{-1}$ exists and is bounded. For every $f$, we have

$$
\begin{aligned}
\left\|\mathcal{K} \mathcal{A}_{\xi, \eta}^{-1} f\right\| & =\left\|\mathcal{A}_{\xi, \eta}^{-1} f\right\| \quad \text { (since } \mathcal{K} \text { is isometric) } \\
& \left.\leq\left\|\mathcal{A}_{\xi, \eta}^{-1}\right\| \cdot\|f\| \quad \text { (since } \mathcal{A}_{\xi, \eta}^{-1} \text { is bounded }\right)
\end{aligned}
$$

which implies, due to the arbitrariness of $f$, that $\mathcal{K} \mathcal{A}_{\xi, \eta}^{-1}$ is bounded. In addition,

$$
\begin{aligned}
W_{\xi, \eta} \mathcal{K} \mathcal{A}_{\xi, \eta}^{-1} & =\mathcal{A}_{\xi, \eta} \mathcal{K} \mathcal{K} \mathcal{A}_{\xi, \eta}^{-1} \quad \text { (by Proposition 4.1.3 } \\
& =\mathcal{A}_{\xi, \eta} \mathcal{A}_{\xi, \eta}^{-1} \\
& =I
\end{aligned}
$$

and

$$
\begin{aligned}
\mathcal{K} \mathcal{A}_{\xi, \eta}^{-1} W_{\xi, \eta} & =\mathcal{K} \mathcal{A}_{\xi, \eta}^{-1} \mathcal{A}_{\xi, \eta} \mathcal{K} \quad \text { (by Proposition 4.1.3) } \\
& =\mathcal{K} \mathcal{K} \\
& =I \quad(\text { since } \mathcal{K} \text { is involutive })
\end{aligned}
$$

These show that $W_{\xi, \eta}^{-1}=\mathcal{K} \mathcal{A}_{\xi, \eta}^{-1}$ is bounded.

The reverse direction is proved by similar arguments.

(4) We have

$$
\mathcal{A}_{\xi, \eta}^{*}=\mathcal{K}^{*} W_{\xi, \eta}^{*}=\mathcal{K} W_{\xi, \eta}^{*}, \quad \mathcal{A}_{\xi, \eta}^{-1}=\mathcal{K}^{-1} W_{\xi, \eta}^{-1}=\mathcal{K} W_{\xi, \eta}^{-1} .
$$

Thus, the operator $\mathcal{A}_{\xi, \eta}$ is unitary if and only if $\mathcal{K} W_{\xi, \eta}^{*}=\mathcal{K} W_{\xi, \eta}^{-1}$, and hence, since $\mathcal{K}$ is involutive, $W_{\xi, \eta}$ is unitary.

The following result shows that a square of an anti-linear weighted composition operator is a linear weighted composition operator, with appropriate symbols.

Proposition 4.1.5. We have $\mathcal{A}_{\xi, \eta}^{2}=W_{\zeta, \vartheta}$, where $\zeta(z)=\xi(z) \overline{\xi(\kappa \eta(z))}$ and $\vartheta(z)=$ $(\kappa \eta)^{2}(z)$. 
Proof. For every $f(z) \in \mathcal{F}^{2}\left(\mathbb{C}^{n}\right)$, we have

$$
\mathcal{A}_{\xi, \eta}^{2} f(z)=\xi(z) \overline{\mathcal{A}_{\xi, \eta} f(\kappa \eta(z))}=\xi(z) \overline{\xi(\kappa \eta(z))} f\left((\kappa \eta)^{2}(z)\right)=W_{\zeta, \vartheta} f(z)
$$

\subsubsection{Bounded, invertible, and unitary properties}

By Theorem 4.1.4 and Proposition 1.4.5, we get immediately the following result for boundedness of an anti-linear weighted composition operator $\mathcal{A}_{\xi, \eta}$.

Proposition 4.1.6. If the anti-linear weighted composition operator $\mathcal{A}_{\xi, \eta}$ is bounded on $\mathcal{F}^{2}\left(\mathbb{C}^{n}\right)$, then there exists a linear operator $A$ on $\mathbb{C}^{n}$ with $\|A\| \leq 1$ and $b \in \mathbb{C}^{n}$, such that $\eta(z)=A z+b$ and $M(\xi, \eta)<\infty$. In particular, when $A$ is invertible, these two conditions together are sufficient also.

The following result, in the case when $\xi(z)$ has an exponential form (while $\eta(z)$ is affine), provides an important equivalent relationship between a bounded linear weighted composition operator $W_{\xi, \eta}$ and a bounded anti-linear weighted composition operator $\mathcal{A}_{\xi, \eta}$.

Theorem 4.1.7. Let $\eta(z)=A z+b$ and $\xi(z)=e^{\langle z, \beta\rangle}$, where $A$ is a linear operator on $\mathbb{C}^{n}, b, \beta \in \mathbb{C}^{n}$. The following assertions are equivalent:

1. The linear weighted composition operator $W_{\xi, \eta}: \mathcal{F}^{2}\left(\mathbb{C}^{n}\right) \rightarrow \mathcal{F}^{2}\left(\mathbb{C}^{n}\right)$ is bounded.

2. The anti-linear weighted composition operator $\mathcal{A}_{\xi, \eta}: \mathcal{F}^{2}\left(\mathbb{C}^{n}\right) \rightarrow \mathcal{F}^{2}\left(\mathbb{C}^{n}\right)$ is bounded.

3. The linear composition operator $C_{\eta_{\beta}}: \mathcal{F}^{2}\left(\mathbb{C}^{n}\right) \rightarrow \mathcal{F}^{2}\left(\mathbb{C}^{n}\right)$ is bounded. Here, $\eta_{\beta}(z)=\eta(z+\beta)$.

4. $\|A\| \leq 1$ and $\langle A x, b+A \beta\rangle=0$, whenever $x \in \mathbb{C}^{n}$ with $|A x|=|x|$. 
Proof. Since $(1) \Longleftrightarrow(2)$ has been proved in Theorem 4.1 .4 above, while $(3) \Longleftrightarrow$ (4) by Proposition 1.4.4, it suffices to prove $(2) \Longleftrightarrow(3)$.

For each $\beta \in \mathbb{C}^{n}$, we define the operator $U_{\beta}: \mathcal{F}^{2}\left(\mathbb{C}^{n}\right) \rightarrow \mathcal{F}^{2}\left(\mathbb{C}^{n}\right)$ by

$$
U_{\beta} f(z)=k_{\beta}(z) f(z-\beta), \quad f \in \mathcal{F}^{2}\left(\mathbb{C}^{n}\right), z \in \mathbb{C}^{n} .
$$

Note that by Proposition 1.4.7, $U_{\beta}$ is unitary on $\mathcal{F}^{2}\left(\mathbb{C}^{n}\right)$. If $f \in \mathcal{F}^{2}\left(\mathbb{C}^{n}\right)$, then by Proposition 4.1.3, we have

$$
\begin{aligned}
C_{\eta_{\beta}} \mathcal{K} f(z) & =\mathcal{A}_{\mathbf{1}, \eta_{\beta}} f(z)=\overline{f\left(\kappa \eta_{\beta}(z)\right)}=e^{-|\beta|^{2} / 2} k_{-\beta}(z) \xi(z+\beta) \overline{f\left(\kappa \eta_{\beta}(z)\right)} \\
& =e^{-|\beta|^{2} / 2} k_{-\beta}(z) \mathcal{A}_{\xi, \eta} f(z+\beta)=e^{-|\beta|^{2} / 2} U_{-\beta} \mathcal{A}_{\xi, \eta} f(z)
\end{aligned}
$$

Since $U_{-\beta}$ is unitary and $\mathcal{K}$ is a conjugation, the above relation shows that $\mathcal{A}_{\xi, \eta}$ is bounded if and only if so is $C_{\eta_{\beta}}$.

Identity (1.8) tells us the action of the adjoint of a linear weighted composition operator on kernel functions. Now we investigate this action for anti-linear weighted composition operators.

Lemma 4.1.8. If the anti-linear weighted composition operator $\mathcal{A}_{\xi, \eta}$ is bounded on $\mathcal{F}^{2}\left(\mathbb{C}^{n}\right)$, then

$$
\mathcal{A}_{\xi, \eta}^{*} K_{z}=\xi(z) K_{\kappa \eta(z)}, \quad z \in \mathbb{C}^{n} .
$$

Proof. By Proposition 4.1.3, we have

$$
\begin{aligned}
\mathcal{A}_{\xi, \eta}^{*} K_{z} & \left.=\mathcal{K} W_{\xi, \eta}^{*} K_{z}=\mathcal{K}\left(\overline{\xi(z)} K_{\eta(z)}\right) \quad \text { (by }(1.8)\right) \\
& =\xi(z) \mathcal{K}\left(K_{\eta(z)}\right) \quad(\text { since } \mathcal{K} \text { is anti-linear) }
\end{aligned}
$$

Moreover, for each $u \in \mathbb{C}^{n}$,

$$
\begin{aligned}
\mathcal{K}\left(K_{\eta(z)}\right)(u) & =\overline{K_{\eta(z)}(\kappa(u))}=e^{\langle\eta(z), \kappa(u)\rangle} \\
& =e^{\langle u, \kappa \eta(z)\rangle}=K_{\kappa \eta(z)}(u)
\end{aligned}
$$

Hence, $\mathcal{A}_{\xi, \eta}^{*} K_{z}=\xi(z) K_{\kappa \eta(z)}$. 
Next, we investigate an invertibility of bounded anti-linear weighted composition operators on $\mathcal{F}^{2}\left(\mathbb{C}^{n}\right)$. Analyzing the proof of Zhao, we observe that an invertible operator $Q$ in Proposition $1.4 .5(2)$ satisfies $\left\|Q^{-1}\right\|=1$. Also such a square matrix must necessarily be unitary. This phenomenon is easily generalized to a general situation.

Lemma 4.1.9. A bounded linear operator $U$, acting on a Hilbert space $\mathcal{H}$, is unitary if and only if $U$ is invertible with $\|U\|=\left\|U^{-1}\right\|=1$.

Proof. • For the necessity, suppose that $U$ is unitary, i.e. $U U^{*}=U^{*} U=I$. This means that $U$ is invertible with $U^{-1}=U^{*}$. We have

$$
\begin{aligned}
\|U x\|^{2} & =\langle U x, U x\rangle=\left\langle x, U^{*} U x\right\rangle \quad \text { (the adjoint of a linear operator) } \\
& =\langle x, x\rangle=\|x\|^{2}, \quad \forall x \in \mathcal{H},
\end{aligned}
$$

which implies, due to the arbitrariness of $x$, that $\|U\|=1$. Meanwhile, for the adjoint $U^{*}$, we see

$$
\begin{aligned}
\left\|U^{*} x\right\|^{2} & =\left\langle U^{*} x, U^{*} x\right\rangle=\left\langle x, U U^{*} x\right\rangle \quad \text { (the adjoint of a linear operator) } \\
& =\langle x, x\rangle=\|x\|^{2}, \quad \forall x \in \mathcal{H}
\end{aligned}
$$

which gives $\left\|U^{-1}\right\|=\left\|U^{*}\right\|=1$.

- For the sufficiency, suppose that $U$ is invertible with $\|U\|=\left\|U^{-1}\right\|=1$. For every $x \in \mathcal{H}$, we have

$$
\|U x\| \leq\|U\| \cdot\|x\|=\|x\|=\left\|U^{-1} U x\right\| \leq\left\|U^{-1}\right\| \cdot\|U x\|=\|U x\|,
$$

which implies that $U$ is isometric. By the polarization identity 1.1 , we get

$$
\langle U x, U y\rangle=\langle x, y\rangle, \quad \forall x, y \in \mathcal{H}
$$

Moreover, by definition of the adjoint of a linear operator,

$$
\langle U x, U y\rangle=\left\langle x, U^{*} U y\right\rangle
$$

Thus, $U^{*} U=I$, which gives $U^{-1}=U^{*}$. The proof is complete. 
Lemma 4.1 .9 allows us to precise the criterion of a bounded invertible weighted composition operators on $\mathcal{F}^{2}\left(\mathbb{C}^{n}\right)$.

Proposition 4.1.10. The following statements are equivalent:

1. The linear weighted composition operator $W_{\xi, \eta}$ is invertible on $\mathcal{F}^{2}\left(\mathbb{C}^{n}\right)$.

2. The anti-linear weighted composition operator $\mathcal{A}_{\xi, \eta}$ is invertible on $\mathcal{F}^{2}\left(\mathbb{C}^{n}\right)$.

3. The functions $\xi, \eta$ are of forms:

$$
\eta(z)=A z+b, \quad \text { and } \quad \xi(z)=c e^{-\langle A z, b\rangle}, \quad z \in \mathbb{C}^{n}
$$

where $A$ is a unitary linear operator on $\mathbb{C}^{n}, b \in \mathbb{C}^{n}$, and $c \in \mathbb{C} \backslash\{0\}$.

Proof. By Theorem 4.1.4, we have (1) $\Longleftrightarrow(2)$. Now we prove that $(1) \Longleftrightarrow(3)$.

- Suppose $W_{\psi, \varphi}$ is bounded invertible on $\mathcal{F}^{2}\left(\mathbb{C}^{n}\right)$. By Proposition 1.4 .5 and Lemma 4.1.9, $\varphi(z)=A z+b$, where $A$ is a linear unitary operator on $\mathbb{C}^{n}$ and $b \in \mathbb{C}^{n}$. By Lemma 1.4.6, we have the form 4.1 for $\xi$, where $c \neq 0$.

- Conversely, suppose $\xi, \eta$ are of forms 4.1. We have

$$
\begin{aligned}
M_{z}(\xi, \eta) & =\left|c e^{-\langle A z, b\rangle}\right|^{2} e^{|A z+b|^{2}-|z|^{2}}=|c|^{2} e^{-2 \operatorname{Re}\langle A z, b\rangle} e^{|A z+b|^{2}-|z|^{2}} \\
& \left.=|c|^{2} e^{-2 \operatorname{Re}\langle A z, b\rangle} e^{|A z|^{2}+|b|^{2}+2 \operatorname{Re}\langle A z, b\rangle-|z|^{2}} \quad(\text { by } 2.1]\right) \\
& =|c|^{2} e^{|A z|^{2}-|z|^{2}+|b|^{2}}=|c|^{2} e^{|b|^{2}} \quad \text { (since } A \text { is unitary) }
\end{aligned}
$$

which shows that $0<m(\xi, \eta)=M(\xi, \eta)<\infty$. By Proposition 1.4.5, the operator $W_{\xi, \eta}$ is bounded invertible on the Fock space $\mathcal{F}^{2}\left(\mathbb{C}^{n}\right)$.

Remark 4.1.11. Since involutivity is a particular type of invertibility, the symbols that induce an involutive bounded weighted composition operator on $\mathcal{F}^{2}\left(\mathbb{C}^{n}\right)$, should have form 4.1). So, Proposition 4.1.10 is useful in characterizing involutivity of both linear and anti-linear weighted composition operators. 
The last task of this part is to study a unitary property of bounded anti-linear weighted composition operators on $\mathcal{F}^{2}\left(\mathbb{C}^{n}\right)$.

Proposition 4.1.12. The following statements are equivalent.

1. The anti-linear weighted composition operator $\mathcal{A}_{\xi, \eta}$ is unitary on $\mathcal{F}^{2}\left(\mathbb{C}^{n}\right)$.

2. The linear weighted composition operator $W_{\xi, \eta}$ is unitary on $\mathcal{F}^{2}\left(\mathbb{C}^{n}\right)$.

3. The linear weighted composition operator $W_{\xi, \eta}$ is isometric on $\mathcal{F}^{2}\left(\mathbb{C}^{n}\right)$.

4. The anti-linear weighted composition operator $\mathcal{A}_{\xi, \eta}$ is isometric on $\mathcal{F}^{2}\left(\mathbb{C}^{n}\right)$.

5. The functions $\xi$ and $\eta$ are of forms

$$
\eta(z)=A z+b, \xi(z)=c e^{-\langle A z, b\rangle}, \quad z \in \mathbb{C}^{n}
$$

where $A$ is an unitary linear operator on $\mathbb{C}^{n}, b \in \mathbb{C}^{n}$, and $c \in \mathbb{C}$, with

$$
|c|^{2} e^{|b|^{2}}=1
$$

Proof. By Proposition 1.4.7, (2) $\Longleftrightarrow(3) \Longleftrightarrow(5)$, while by Theorem 4.1.4. (1) $\Longleftrightarrow(2)$ and $(3) \Longleftrightarrow(4)$.

\subsubsection{Selfadjoint property}

We characterize selfadjointness of bounded anti-linear weighted composition operators on $\mathcal{F}^{2}\left(\mathbb{C}^{n}\right)$. Since the span of kernel functions is dense in $\mathcal{F}^{2}\left(\mathbb{C}^{n}\right)$, by Lemma 1.1.7, our problem is reduced to finding $\xi$ and $\eta$ such that

$$
\mathcal{A}_{\xi, \eta}^{*} K_{z}=\mathcal{A}_{\xi, \eta} K_{z}, \quad \forall z \in \mathbb{C}^{n}
$$

It turns out that in this case, the matrix $A$ appeared in the formula of the function $\eta$ in Proposition 4.1.6, is complex symmetric with the standard conjugation $\kappa$. 
Proposition 4.1.13. The following statements are equivalent:

1. The bounded linear weighted composition operator $W_{\xi, \eta}=\mathcal{A}_{\xi, \eta} \mathcal{K}$ is $\mathcal{K}$-symmetric on $\mathcal{F}^{2}\left(\mathbb{C}^{n}\right)$.

2. The bounded anti-linear weighted composition operator $\mathcal{A}_{\xi, \eta}$ is selfadjoint on $\mathcal{F}^{2}\left(\mathbb{C}^{n}\right)$

3. The functions $\xi$ and $\eta$ are of forms

$$
\eta(z)=A z+b, \quad \text { and } \quad \xi(z)=c e^{\langle z, \kappa b\rangle}, \quad z \in \mathbb{C}^{n}
$$

where $A$ is a linear operator on $\mathbb{C}^{n}, b \in \mathbb{C}^{n}$, and $c \in \mathbb{C} \backslash\{0\}$, such that

$$
\|A\| \leq 1, \quad\langle A x, b+A \kappa b\rangle=0, \quad \text { whenever } x \in \mathbb{C}^{n} \text { with }|A x|=|x|,
$$

and

$$
\kappa A \kappa=A^{*}
$$

Proof. By Proposition 4.1.3, we have $\mathcal{A}_{\xi, \eta}=W_{\xi, \eta} \mathcal{K}$, and hence,

$$
\mathcal{A}_{\xi, \eta}^{*}=\mathcal{K}^{*} W_{\xi, \eta}^{*}=\mathcal{K} W_{\xi, \eta}^{*}
$$

These equations clearly imply that $(1) \Longleftrightarrow(2)$.

We now prove $(2) \Longleftrightarrow(3)$. Note that a boundedness of $\mathcal{A}_{\xi, \eta}$ is equivalent to (4.3), by Theorem 4.1.7.

- Suppose that $\mathcal{A}_{\xi, \eta}$ is selfadjoint, that is, $\mathcal{A}_{\xi, \eta}^{*}=\mathcal{A}_{\xi, \eta}$. For arbitrary $z, u \in \mathbb{C}^{n}$, by Lemma 4.1.8, we have

$$
\xi(z) K_{\kappa \eta(z)}(u)=\mathcal{A}_{\xi, \eta}^{*} K_{z}(u)=\mathcal{A}_{\xi, \eta} K_{z}(u)=\xi(u) \overline{K_{z}(\kappa \eta(u))},
$$

which gives

$$
\xi(z) e^{\langle u, \kappa \eta(z)\rangle}=\xi(u) e^{\langle z, \kappa \eta(u)\rangle}
$$


Since $\eta(z)=A z+b$, this equality is rewritten as

$$
\xi(z) e^{\langle u, \kappa A z+\kappa b\rangle}=\xi(u) e^{\langle z, \kappa A u+\kappa b\rangle} .
$$

Letting $u=0$, we get the form of $\xi(z)$ in 4.2 , with $c=\xi(0)$. Substituting $\xi(z)$ back into 4.5), we have

$$
c e^{\langle z+u, \kappa b\rangle+\langle u, \kappa A z\rangle}=\xi(z) e^{\langle u, \kappa A z+\kappa b\rangle}=\xi(u) e^{\langle z, \kappa A u+\kappa b\rangle}=c e^{\langle z+u, \kappa b\rangle+\langle z, \kappa A u\rangle},
$$

and consequently,

$$
\langle u, \kappa A z\rangle=\langle z, \kappa A u\rangle, \quad \forall z, u \in \mathbb{C}^{n} .
$$

Since $\kappa$ is a conjugation on $\mathbb{C}^{n}$, we see

$$
\langle u, \kappa A z\rangle=\langle A z, \kappa u\rangle
$$

Thus, we have

$$
\langle A z, \kappa u\rangle=\langle z, \kappa A u\rangle=\langle z,(\kappa A \kappa) \kappa u\rangle, \quad \forall z, u \in \mathbb{C}^{n}
$$

Equivalently,

$$
\langle A z, w\rangle=\langle z,(\kappa A \kappa) w\rangle, \quad \forall z, w \in \mathbb{C}^{n},
$$

which means that $A^{*} w=(\kappa A \kappa) w, \forall w \in \mathbb{C}^{n}$, and so it holds that 4.4.

- Conversely, suppose that conditions 4.2 4.4 hold. We have

$$
\begin{aligned}
\mathcal{A}_{\xi, \eta} K_{z}(u) & =\xi(u) \overline{K_{z}(\kappa \eta(u))}=\xi(u) e^{\langle z, \kappa \eta(u)\rangle} \\
& =c e^{\langle z+u, \kappa b\rangle+\langle z, \kappa A u\rangle}=c e^{\langle z+u, \kappa b\rangle+\langle A u, \kappa z\rangle} \\
& =c e^{\langle z+u, \kappa b\rangle+\left\langle u, A^{*} \kappa z\right\rangle} .
\end{aligned}
$$

By (4.4), we see

$$
\begin{aligned}
\mathcal{A}_{\xi, \eta} K_{z}(u) & =c e^{\langle z+u, \kappa b\rangle+\langle u, \kappa A z\rangle}=c e^{\langle z, \kappa b\rangle+\langle u, \kappa b+\kappa A z\rangle} \\
& =\xi(z) e^{\langle u, \kappa \eta(z)\rangle}=\xi(z) K_{\kappa \eta(z)}(u) \\
& =\mathcal{A}_{\xi, \eta}^{*} K_{z}(u) \quad \text { (by Lemma 4.1.8), }
\end{aligned}
$$


which gives

$$
\mathcal{A}_{\xi, \eta}^{*} K_{z}=\mathcal{A}_{\xi, \eta} K_{z}, \quad \forall z \in \mathbb{C}^{n}
$$

Since the span of the kernel functions is dense in $\mathcal{F}^{2}\left(\mathbb{C}^{n}\right)$, also $\mathcal{A}_{\xi, \eta}^{*}$ and $\mathcal{A}_{\xi, \eta}$ are bounded, we have $\mathcal{A}_{\xi, \eta}^{*}=\mathcal{A}_{\xi, \eta}$. This shows that the operator $\mathcal{A}_{\xi, \eta}$ is selfadjoint.

Remark 4.1.14. Proposition 4.1.13 provides the structure of bounded linear weighted composition operators, which are $\mathcal{K}$-symmetric on $\mathcal{F}^{2}\left(\mathbb{C}^{n}\right)$. In next section, we give another class of complex symmetric bounded linear weighted composition operators, which contains properly $\mathcal{K}$-symmetric operators in Proposition 4.1 .13 .

\subsubsection{Conjugation}

Before giving the structure of weighted composition conjugations, we prove the following lemma:

Lemma 4.1.15. An anti-linear mapping is a conjugation if and only if it is both unitary and selfadjoint.

Proof. • Suppose that $\mathcal{C}$ is a conjugation. Then it is both isometric and involutive.

We have

$$
\left\langle\mathcal{C}^{*} \mathcal{C} y, x\right\rangle=\langle\mathcal{C} x, \mathcal{C} y\rangle=\langle y, x\rangle, \quad \forall x, y \in \mathcal{H}
$$

which implies, due to the arbitrariness of $x$ and $y$, that $\mathcal{C}^{*} \mathcal{C}=I$. Since $\mathcal{C}$ is involutive, we have $\mathcal{C}=\mathcal{C}^{-1}$, and so, $\mathcal{C}=\mathcal{C}^{-1}=\mathcal{C}^{*}$.

- Conversely, suppose that $\mathcal{C}$ is both unitary and selfadjoint, that is $\mathcal{C}^{-1}=\mathcal{C}^{*}=$

$\mathcal{C}$. Clearly, $\mathcal{C}$ must be involutive, and moreover, it is isometric since

$$
\begin{aligned}
\|\mathcal{C} x\|^{2} & =\langle\mathcal{C} x, \mathcal{C} x\rangle=\left\langle\mathcal{C}^{*} \mathcal{C} x, x\right\rangle \\
& =\langle x, x\rangle=\|x\|^{2}, \quad \forall x \in \mathcal{H}
\end{aligned}
$$


Combining Propositions 4.1.12,4.1.13 gives the following important criterion.

Theorem 4.1.16. The anti-linear weighted composition operator $\mathcal{A}_{\xi, \eta}: \mathcal{F}^{2}\left(\mathbb{C}^{n}\right) \rightarrow$ $\mathcal{F}^{2}\left(\mathbb{C}^{n}\right)$ is a conjugation if and only if

$$
\eta(z)=A z+b, \quad \text { and } \quad \xi(z)=c e^{-\langle A z, b\rangle}, \quad z \in \mathbb{C}
$$

where $A$ is a linear operator on $\mathbb{C}^{n}, b \in \mathbb{C}^{n}$, and $c \in \mathbb{C}$ that satisfy the following conditions

$$
\begin{gathered}
A^{-1}=A^{*}=\kappa A \kappa, \\
A \kappa b+b=0, \\
|c|^{2} e^{|b|^{2}}=1 .
\end{gathered}
$$

\subsubsection{Involutive property}

In this subsection, we investigate an involutivity of anti-linear weighted composition operators. As for the case of one dimension, an involutive bounded anti-linear weighted composition operator must necessarily be a conjugation on the Fock space $\mathcal{F}^{2}\left(\mathbb{C}^{n}\right)$.

Theorem 4.1.17. Suppose that $\mathcal{A}_{\xi, \eta}$ is a bounded anti-linear weighted composition operator. The following statements are equivalent:

1. $\mathcal{A}_{\xi, \eta}$ is involutive.

2. $\mathcal{A}_{\xi, \eta}$ is a conjugation.

3. The functions $\xi, \eta$ are of forms (4.6) with conditions (4.7)-(4.9).

Proof. Clearly, $(2) \Longleftrightarrow(3)$ by Theorem 4.1.16.

Note that by Proposition 4.1.5, the operator $\mathcal{A}_{\xi, \eta}$ is involutive if and only if

$$
\mathcal{A}_{\xi, \eta}^{2} f(z)=\xi(z) \overline{\xi(\kappa \eta(z))} f\left((\kappa \eta)^{2}(z)\right)=f(z), \quad \forall f \in \mathcal{F}^{2}\left(\mathbb{C}^{n}\right) .
$$


Since $\mathcal{A}_{\xi, \eta}$ is bounded on $\mathcal{F}^{2}\left(\mathbb{C}^{n}\right)$, by Proposition 4.1.6. $\eta(z)=A z+b$, and hence,

$$
(\kappa \eta)^{2}(z)=(\kappa A)^{2} z+\kappa A \kappa b+\kappa b .
$$

With the help of identities $4.10-4.11$, we prove $(1) \Longleftrightarrow(3)$ as follows.

- Suppose that $\mathcal{A}_{\xi, \eta}$ is involutive, which gives 4.10 . In particular, for $f(z) \equiv$ $\mathbf{1} \in \mathcal{F}^{2}\left(\mathbb{C}^{n}\right)$, we get

$$
\xi(z) \overline{\xi(\kappa \eta(z))}=1
$$

Then equation (4.10) is reduced to

$$
f\left((\kappa \eta)^{2}(z)\right)=f(z), \forall f \in \mathcal{F}^{2}\left(\mathbb{C}^{n}\right) .
$$

Now taking $f(z) \equiv z$ in 4.13 , we have

$$
(\kappa \eta)^{2}(z)=z, \forall z \in \mathbb{C}^{n},
$$

which implies, by 4.11, that

$$
(\kappa A)^{2} z+\kappa A \kappa b+\kappa b=z, \forall z \in \mathbb{C}^{n},
$$

from which (4.7) and (4.8) follow.

Note that an involutive bounded operator is also invertible. Then by Proposition 4.1.10, we have 4.6), where the linear operator $A$ is unitary on $\mathbb{C}^{n}$. Since $\xi(z)=c e^{-\langle A z, b\rangle}$, we get

$$
\begin{aligned}
\xi(\kappa \eta(z)) & =c e^{-\langle A \kappa \eta(z), b\rangle}=c e^{-\langle A \kappa A z+A \kappa b, b\rangle} \\
& \left.=c e^{-\langle\kappa z+A \kappa b, b\rangle} \quad(\text { by } 4.7)\right) \\
& \left.=c e^{-\langle\kappa z-b, b\rangle} \quad(\text { by } 4.8)\right) \\
& =c e^{|b|^{2}} e^{-\langle\kappa z, b\rangle},
\end{aligned}
$$

which gives

$$
\begin{aligned}
\xi(z) \overline{\xi(\kappa \eta(z))} & =c e^{-\langle A z, b\rangle} \bar{c} e^{|b|^{2}} e^{-\langle b, \kappa z\rangle}=|c|^{2} e^{|b|^{2}} e^{-\langle A z, b\rangle-\langle b, \kappa z\rangle} \\
& =|c|^{2} e^{|b|^{2}} e^{-\left\langle z, A^{*} b\right\rangle-\langle b, \kappa z\rangle}=|c|^{2} e^{|b|^{2}} e^{-\left\langle z, A^{*} b\right\rangle-\langle z, \kappa b\rangle} \\
& =|c|^{2} e^{|b|^{2}} e^{-\left\langle z, A^{*} b+\kappa b\right\rangle}
\end{aligned}
$$


Hence, equation 4.12) becomes

$$
|c|^{2} e^{|b|^{2}} e^{-\left\langle z, A^{*} b+\kappa b\right\rangle}=1, \quad \forall z \in \mathbb{C}^{n} .
$$

In particular, for $z=0$, we get 4.9 .

- Conversely, suppose conditions (4.6)- 4.9 hold. We can rewrite

$$
\xi(z)=c e^{\left\langle z,-A^{*} b\right\rangle} .
$$

For every $x \in \mathbb{C}^{n}$ with $|A x|=|x|$, we have

$$
\left\langle A x, b+A\left(-A^{*} b\right)\right\rangle=\langle A x, b-b\rangle=0 .
$$

Thus, by Theorem 4.1.7, $\mathcal{A}_{\xi, \eta}$ is bounded.

To show that $\mathcal{A}_{\xi, \eta}$ is involutive, we use 4.10 . Indeed, we take arbitrarily $f(z) \in \mathcal{F}^{2}\left(\mathbb{C}^{n}\right)$, and observe that

$$
\begin{aligned}
\xi(z) \overline{\xi(\kappa \eta(z))} & =|c|^{2} e^{-\langle A z, b\rangle-\langle b, A \kappa A z+A \kappa b\rangle}=|c|^{2} e^{-\langle A z, b\rangle-\langle b, \kappa z+A \kappa b\rangle} \quad \text { (by (4.7)) } \\
& =|c|^{2} e^{-\left\langle z, A^{*} b\right\rangle-\langle b, \kappa z\rangle-\langle b, A \kappa b\rangle}=|c|^{2} e^{-\left\langle z, A^{*} b+\kappa b\right\rangle-\langle b, A \kappa b\rangle} \quad \text { (by (1.2)) } \\
& =|c|^{2} e^{-\left\langle z, A^{*} b+\kappa b\right\rangle-\langle b,-b\rangle}=|c|^{2} e^{|b|^{2}} e^{-\langle z, \kappa A \kappa b+\kappa b\rangle} \quad \text { (by (4.7)) } \\
& =1 \quad(\text { by }(4.8)-(4.9)) .
\end{aligned}
$$

On the other hand, by 4.11, we have

$$
\begin{aligned}
(\kappa \eta)^{2}(z) & =(\kappa A)^{2} z+\kappa(A \kappa b+b) \\
& =z+\kappa(A \kappa b+b) \quad(\text { by } 4.7)) \\
& =z \quad(\text { by } 4.8)) .
\end{aligned}
$$

Thus,

$$
\mathcal{A}_{\xi, \eta}^{2} f(z)=\xi(z) \overline{\xi(\kappa \eta(z))} f\left((\kappa \eta)^{2}(z)\right)=f(z), \forall z \in \mathbb{C}^{n}
$$

which show that $\mathcal{A}_{\xi, \eta}$ is involutive. 


\subsection{Linear weighted composition operators}

In this section, we consider a bounded linear weighted composition operator $W_{\psi, \varphi}$, and study its complex symmetry in the Fock space $\mathcal{F}^{2}\left(\mathbb{C}^{n}\right)$. Note that a bounded operator $W_{\psi, \varphi}$ gives $\varphi(z)=Q z+p$ with $\|Q\| \leq 1$.

\subsubsection{Complex symmetry with weighted composition con- jugations}

In what follows, the weighted composition conjugation on $\mathcal{F}^{2}\left(\mathbb{C}^{n}\right)$ is denoted by $\mathcal{C}_{A, b, c}$, that is

$$
\mathcal{C}_{A, b, c} f(z):=c e^{\langle z, \kappa b\rangle} \overline{f(\kappa(A z+b))}
$$

where $A, b, c$ satisfy conditions of Theorem 4.1.16. Clearly, with $A=I_{n}, b=0, c=$ 1 , we get the standard conjugation $\mathcal{K}$.

We consider bounded linear weighted composition operators, and study under what conditions, these operators are $\mathcal{C}_{A, b, c}$-symmetric.

We have the following important criterion.

Theorem 4.2.1. Let $\mathcal{C}_{A, b, c}$ be a weighted composition conjugation on $\mathcal{F}^{2}\left(\mathbb{C}^{n}\right)$, defined by 4.14) (that is conditions (4.7) - 4.9 hold). Let further, $W_{\psi, \varphi}$ be a linear weighted composition operator induced by $\psi \in \operatorname{Hol}\left(\mathbb{C}^{n}\right)$ and $\varphi \in \operatorname{HSel}\left(\mathbb{C}^{n}\right)$. Then the operator $W_{\psi, \varphi}$ is $\mathcal{C}_{A, b, c}$-symmetric on $\mathcal{F}^{2}\left(\mathbb{C}^{n}\right)$ if and only if the following conditions hold:

$$
\varphi(z)=Q z+p, \text { and } \psi(z)=\psi(0) e^{\langle z, q\rangle}
$$

where $Q$ is a linear operator on $\mathbb{C}^{n}, p, q \in \mathbb{C}^{n}$, with $q=\kappa A p+\kappa b-Q^{*} \kappa b$, such that

$$
\begin{gathered}
(A Q)^{*}=\kappa A Q \kappa, \\
\|Q\| \leq 1, \text { and }\langle Q x, p+Q q\rangle=0 \text { whenever } x \in \mathbb{C}^{n} \text { with }|Q x|=|x| .
\end{gathered}
$$


Proof. Since $W_{\psi, \varphi}$ is bounded, by Proposition 1.4.5, there exist a linear operator $Q: \mathbb{C}^{n} \rightarrow \mathbb{C}^{n}$ with $\|Q\| \leq 1$, and a vector $p \in \mathbb{C}^{n}$ such that $\varphi(z)=Q z+p$.

- Suppose $W_{\psi, \varphi}$ is $\mathcal{C}_{A, b, c^{-}}$symmetric on $\mathcal{F}^{2}\left(\mathbb{C}^{n}\right)$. This implies, in particular, that

$$
\mathcal{C}_{A, b, c} W_{\psi, \varphi}^{*} K_{z}(u)=W_{\psi, \varphi} \mathcal{C}_{A, b, c} K_{z}(u), \quad \forall z, u \in \mathbb{C}^{n}
$$

On one hand, we have

$$
\begin{aligned}
\mathcal{C}_{A, b, c} W_{\psi, \varphi}^{*} K_{z}(u) & =\mathcal{C}_{A, b, c}\left(\overline{\psi(z)} K_{\varphi(z)}\right)(u) \quad(\text { by }(1.8)) \\
& =\psi(z)\left(\mathcal{C}_{A, b, c} K_{\varphi(z)}\right)(u) \quad\left(\text { since } \mathcal{C}_{A, b, c}\right. \text { is anti-linear) } \\
& =c \psi(z) e^{\langle u, \kappa b\rangle+\langle Q z+p, \kappa(A u+b)\rangle} .
\end{aligned}
$$

On the other hand, since $\mathcal{C}_{A, b, c} K_{z}(u)=c e^{\langle u, \kappa b\rangle} \overline{K_{z}(\kappa(A u+b))}$, the right-hand side of equation 4.18 is rewritten as

$$
W_{\psi, \varphi} \mathcal{C}_{A, b, c} K_{z}(u)=\psi(u)\left(\mathcal{C}_{A, b, c} K_{z}\right)(\varphi(u))=\psi(u) c e^{\langle Q u+p, \kappa b\rangle+\langle z, \kappa A Q u+\kappa A p+\kappa b\rangle} .
$$

From these, identity 4.18 is reduced to

$$
\psi(z) e^{\langle u, \kappa b\rangle+\langle Q z+p, \kappa(A u+b)\rangle}=\psi(u) e^{\langle Q u+p, \kappa b\rangle+\langle z, \kappa A Q u+\kappa A p+\kappa b\rangle} .
$$

Letting $u=0$ in this equation, we get

$$
\psi(z) e^{\langle Q z+p, \kappa b\rangle}=\psi(0) e^{\langle p, \kappa b\rangle+\langle z, \kappa A p+\kappa b\rangle},
$$

which gives 4.15) with $q=\kappa A p+\kappa b-Q^{*} \kappa b$.

Now we substitute $\psi$ back in both sides of 4.19 to obtain

$$
\begin{aligned}
& \langle Q z, \kappa A u\rangle+\langle u, \kappa b\rangle+\langle p, \kappa A u\rangle+\langle z, q\rangle+\langle Q z, \kappa b\rangle \\
& =\langle z, \kappa A Q u\rangle+\langle u, q\rangle+\langle Q u, \kappa b\rangle+\langle z, \kappa A p+\kappa b\rangle, \quad \forall z, u \in \mathbb{C}^{n} .
\end{aligned}
$$

Note that

$$
\begin{aligned}
\langle u, \kappa b\rangle+\langle p, \kappa A u\rangle & =\langle u, \kappa b\rangle+\langle A u, \kappa p\rangle=\langle u, \kappa b\rangle+\left\langle u, A^{*} \kappa p\right\rangle \\
& =\left\langle u, \kappa b+A^{*} \kappa p\right\rangle=\left\langle u, q+Q^{*} \kappa b\right\rangle \\
& =\langle u, q\rangle+\left\langle u, Q^{*} \kappa b\right\rangle=\langle u, q\rangle+\langle Q u, \kappa b\rangle,
\end{aligned}
$$


while

$$
\begin{aligned}
\langle z, q\rangle+\langle Q z, \kappa b\rangle & =\langle z, q\rangle+\left\langle z, Q^{*} \kappa b\right\rangle \\
& =\left\langle z, q+Q^{*} \kappa b\right\rangle \\
& =\langle z, \kappa A p+\kappa b\rangle .
\end{aligned}
$$

Then equation 4.20 is reduced to

$$
\langle Q z, \kappa A u\rangle=\langle z, \kappa A Q u\rangle, \quad \forall z, u \in \mathbb{C}^{n}
$$

which implies that $Q^{*} \kappa A u=\kappa A Q u, \forall u \in \mathbb{C}^{n}$, that is $Q^{*} \kappa A=\kappa A Q$. So,

$$
(A Q)^{*}=Q^{*} A^{*}=Q^{*} \kappa A \kappa=\kappa A Q \kappa,
$$

which also means that $A Q$ is $\kappa$-symmetric on $\mathbb{C}^{n}$.

Finally, 4.17) follows from a boundedness of $W_{\psi, \varphi}$, by Theorem 4.1.7.

- Conversely, suppose that conditions 4.15 - 4.17 hold. In this case, by Theorem 4.1.7, the weighted composition operator $W_{\psi, \varphi}$ induced by $\psi$ and $\varphi$ is bounded on $\mathcal{F}^{2}\left(\mathbb{C}^{n}\right)$, and its adjoint operator $W_{\psi, \varphi}^{*}$ is also bounded on $\mathcal{F}^{2}\left(\mathbb{C}^{n}\right)$.

We have

$$
\begin{aligned}
W_{\psi, \varphi} \mathcal{C}_{A, b, c} K_{z}(u) & =\psi(u)\left(\mathcal{C}_{A, b, c} K_{z}\right)(\varphi(u))=\psi(u) c e^{\langle\varphi(u), \kappa b\rangle+\langle z, \kappa A \varphi(u)+\kappa b\rangle} \\
& \left.=c \psi(0) e^{\langle u, q\rangle+\langle Q u+p, \kappa b\rangle+\langle z, \kappa A Q u+\kappa A p+\kappa b\rangle} \quad(\text { by } 4.15)\right) \\
& =c \psi(0) e^{\langle z, \kappa A Q u\rangle+\langle z, \kappa A p+\kappa b\rangle+\left\langle u, q+Q^{*} \kappa b\right\rangle+\langle p, \kappa b\rangle} .
\end{aligned}
$$

By 4.16), we see

$$
\begin{aligned}
\langle z, \kappa A Q u\rangle & =\left\langle z,(A Q)^{*} \kappa u\right\rangle=\left\langle z, Q^{*} A^{*} \kappa u\right\rangle \\
& =\left\langle Q z, A^{*} \kappa u\right\rangle=\langle Q z, \kappa A u\rangle
\end{aligned}
$$

while

$$
\langle z, \kappa A p+\kappa b\rangle=\left\langle z, q+Q^{*} \kappa b\right\rangle, \quad\left(\text { since } q=\kappa A p+\kappa b-Q^{*} \kappa b\right) .
$$


Also, since $q=\kappa A p+\kappa b-Q^{*} \kappa b$, we have

$$
\begin{aligned}
\left\langle u, q+Q^{*} \kappa b\right\rangle & =\langle u, \kappa A p\rangle+\langle u, \kappa b\rangle=\langle A p, \kappa u\rangle+\langle u, \kappa b\rangle \\
& =\left\langle p, A^{*} \kappa u\right\rangle+\langle u, \kappa b\rangle=\langle p, \kappa A u\rangle+\langle u, \kappa b\rangle \quad \text { (by 4.7)), }
\end{aligned}
$$

From these, we can rewrite

$$
\begin{aligned}
W_{\psi, \varphi} \mathcal{C}_{A, b, c} K_{z}(u) & =c \psi(0) e^{\langle Q z, \kappa A u\rangle+\left\langle z, q+Q^{*} \kappa b\right\rangle+\langle p, \kappa A u\rangle+\langle u, \kappa b\rangle+\langle p, \kappa b\rangle} \\
& =c \psi(0) e^{\langle Q z, \kappa A u\rangle+\langle z, q\rangle+\langle Q z, \kappa b\rangle+\langle p, \kappa A u\rangle+\langle u, \kappa b\rangle+\langle p, \kappa b\rangle} \\
& =c \psi(0) e^{\langle z, q\rangle+\langle u, \kappa b\rangle+\langle Q z+p, \kappa A u+\kappa b\rangle} \\
& =\psi(z) c e^{\langle u, \kappa b\rangle+\langle\varphi(z), \kappa(A u+b)\rangle}=\psi(z) c e^{\langle u, \kappa b\rangle} \overline{K_{\varphi(z)}(\kappa(A u+b))} \\
& =\psi(z)\left(\mathcal{C}_{A, b, c} K_{\varphi(z)}\right)(u)=\mathcal{C}_{A, b, c}\left(\overline{\psi(z)} K_{\varphi(z)}\right)(u) \\
& =\mathcal{C}_{A, b, c} W_{\psi, \varphi}^{*} K_{z}(u) \quad(\text { by }(1.8))
\end{aligned}
$$

which gives

$$
\mathcal{C}_{A, b, c} W_{\psi, \varphi}^{*} K_{z}=W_{\psi, \varphi} \mathcal{C}_{A, b, c} K_{z}, \quad \forall z \in \mathbb{C}^{n}
$$

Since the span of the kernel functions is dense in $\mathcal{F}^{2}\left(\mathbb{C}^{n}\right)$, by Lemma 1.1.7. $W_{\psi, \varphi}$ is $\mathcal{C}_{A, b, c}$-symmetric. The proof is complete.

\subsubsection{Normality}

In this subsection, we investigate the normality problem. As mentioned in the Introduction, every normal operator is complex symmetric. So, the question we are interested in is whether all $\mathcal{C}_{A, b, c}$-symmetric weighted composition operators are normal.

Before giving the answer, we study the action of operators $W_{\psi, \varphi} W_{\psi, \varphi}^{*}$ and $W_{\psi, \varphi}^{*} W_{\psi, \varphi}$ on kernel functions. This will be an important step toward studying the normality for $\mathcal{C}_{A, b, c^{-}}$symmetric weighted composition operators. 
Lemma 4.2.2. Let $W_{\psi, \varphi}$ be a $\mathcal{C}_{A, b, c^{-}}$symmetric operator on $\mathcal{F}^{2}\left(\mathbb{C}^{n}\right)$, induced by $\varphi, \psi$ (that is, Theorem 4.2.1 holds). We have

1. $W_{\psi, \varphi} W_{\psi, \varphi}^{*} K_{z}(u)=|\psi(0)|^{2} e^{\langle Q u, Q z\rangle+\left\langle u, q+Q^{*} p\right\rangle+\left\langle q+Q^{*} p, z\right\rangle+|p|^{2}}$.

2. $W_{\psi, \varphi}^{*} W_{\psi, \varphi} K_{z}(u)=|\psi(0)|^{2} e^{\left\langle Q^{*} u, Q^{*} z\right\rangle+\langle u, Q q+p\rangle+\langle p+Q q, z\rangle+|q|^{2}}$.

Proof. We use identity 1.8 and the form of $\psi$ in Theorem 4.2.1.

1) We have

$$
\begin{aligned}
W_{\psi, \varphi} W_{\psi, \varphi}^{*} K_{z}(u) & =W_{\psi, \varphi}\left(\overline{\psi(z)} K_{\varphi(z)}\right)(u)=\overline{\psi(z)} W_{\psi, \varphi}\left(K_{\varphi(z)}\right)(u) \\
& =\overline{\psi(z)} \psi(u) K_{\varphi(z)}(\varphi(u))=\overline{\psi(z)} \psi(u) e^{\langle\varphi(u), \varphi(z)\rangle} \\
& =|\psi(0)|^{2} e^{\langle Q u, Q z\rangle+\left\langle u, q+Q^{*} p\right\rangle+\left\langle q+Q^{*} p, z\right\rangle+|p|^{2}}
\end{aligned}
$$

2) Since

$$
\begin{aligned}
W_{\psi, \varphi} K_{z}(u) & =\psi(u) K_{z}(\varphi(u))=\psi(0) e^{\langle u, q\rangle+\langle\varphi(u), z\rangle} \\
& =\psi(0) e^{\langle p, z\rangle} e^{\left\langle u, q+Q^{*} z\right\rangle}=\psi(0) e^{\langle p, z\rangle} K_{q+Q^{*} z}(u),
\end{aligned}
$$

we have

$$
\begin{aligned}
W_{\psi, \varphi}^{*} W_{\psi, \varphi} K_{z}(u) & =\psi(0) e^{\langle p, z\rangle} W_{\psi, \varphi}^{*} K_{q+Q^{*} z}(u) \\
& =\psi(0) e^{\langle p, z\rangle} \overline{\psi\left(q+Q^{*} z\right)} K_{\varphi\left(q+Q^{*} z\right)}(u) \\
& =|\psi(0)|^{2} e^{\langle p, z\rangle+\left\langle q, q+Q^{*} z\right\rangle+\left\langle u, Q\left(q+Q^{*} z\right)+p\right\rangle} \\
& =|\psi(0)|^{2} e^{\left\langle Q^{*} u, Q^{*} z\right\rangle+\langle u, Q q+p\rangle+\langle p+Q q, z\rangle+|q|^{2}} .
\end{aligned}
$$

With the help of Lemma 4.2.2, we can obtain the following criterion.

Proposition 4.2.3. Let $W_{\psi, \varphi}$ be a $\mathcal{C}_{A, b, c^{-}}$symmetric operator on $\mathcal{F}^{2}\left(\mathbb{C}^{n}\right)$, induced by $\varphi, \psi$ (that is Theorem 4.2.1 holds). Then $W_{\psi, \varphi}$ is normal if and only if $Q$ is normal (that is $Q^{*} Q=Q Q^{*}$ ) and

$$
\left(Q^{*}-I_{n}\right) p=\left(Q-I_{n}\right) q, \quad|p|=|q| .
$$


Proof. Since $W_{\psi, \varphi}$ is bounded, and the span of the kernel functions is dense in $\mathcal{F}^{2}\left(\mathbb{C}^{n}\right)$, the normality of $W_{\psi, \varphi}$ is equivalent to

$$
W_{\psi, \varphi} W_{\psi, \varphi}^{*} K_{u}(z)=W_{\psi, \varphi}^{*} W_{\psi, \varphi} K_{u}(z), \quad \forall u, z \in \mathbb{C}^{n}
$$

By Lemma 4.2.2, the above equation is reduced to

$$
\begin{aligned}
& \langle Q u, Q z\rangle+\left\langle u, q+Q^{*} p\right\rangle+\left\langle q+Q^{*} p, z\right\rangle+|p|^{2} \\
& =\left\langle Q^{*} u, Q^{*} z\right\rangle+\langle u, p+Q q\rangle+\langle p+Q q, z\rangle+|q|^{2}, \forall u, z \in \mathbb{C}^{n} .
\end{aligned}
$$

In particular, for $z=u=0$, we get $|p|=|q|$. Substituting $|p|=|q|$ back into 4.22, we obtain

$$
\begin{aligned}
& \langle Q u, Q z\rangle+\left\langle u, q+Q^{*} p\right\rangle+\left\langle q+Q^{*} p, z\right\rangle \\
& =\left\langle Q^{*} u, Q^{*} z\right\rangle+\langle u, p+Q q\rangle+\langle p+Q q, z\rangle, \forall u, z \in \mathbb{C}^{n} .
\end{aligned}
$$

Now taking $z=0$ in the above equation, we get

$$
\left\langle u, q+Q^{*} p\right\rangle=\langle u, p+Q q\rangle, \quad \forall u \in \mathbb{C}^{n} \Longleftrightarrow q+Q^{*} p=p+Q q .
$$

From these, equation 4.23 is reduced to

$$
\langle Q u, Q z\rangle=\left\langle Q^{*} u, Q^{*} z\right\rangle, \quad \forall z, u \in \mathbb{C}^{n}
$$

This shows that $Q^{*} Q=Q Q^{*}$, that is $Q$ is normal. 



\section{Chapter 5}

\section{Conclusion \& Future Works}

In the thesis, we characterized

- weighted composition operators, which are conjugations (Theorems 2.1 .18 \& 4.1.16,

- weighted composition operators, which are complex symmetric (Theorems $2.2 .8 \& 4.2 .1$,

for Fock spaces in both cases $\mathcal{F}^{2}(\mathbb{C})$ and $\mathcal{F}^{2}\left(\mathbb{C}^{n}\right), n \geq 2$.

The results for $\mathcal{F}^{2}(\mathbb{C})$ allow us to study complex symmetric $C_{0}$-semigroups on this space. Theorem 3.2.4 is a generalization of Stone's theorem. Meanwhile, Theorem 3.5.5 characterizes a semigroup of weighted composition operators, when it is a complex symmetric $C_{0}$-semigroup on $\mathcal{F}^{2}(\mathbb{C})$ with respect to some weighted composition operator.

Basing on the obtained results, we raise some open problems, for a future study.

\subsection{Complex symmetry in other function spaces}

The first problem we are interested in is to study the complex symmetry in other function spaces, such as Hardy spaces, Bergman spaces, Dirichlet spaces, etc. By 
the methods similar to those used in the thesis, we believe that this problem can be solved. We can completely study the structures of weighted composition conjugations as well as complex symmetric $C_{0}$-semigroups on these functions spaces.

\subsection{Complex symmetry of unbounded operators}

Note that all problems considered in this thesis are for bounded weighted composition operators. A very natural question to ask is: how about unbounded operators? To the best of our knowledge, the problems of unbounded weighted composition operators have never been treated before.

In view of Definition 1.1.8, for unbounded operators, every $\mathcal{C}$-selfadjoint operator is $\mathcal{C}$-symmetric, but the converse implication may not be true. In fact, the class of $\mathcal{C}$-symmetric operators is much bigger than the $\mathcal{C}$-selfadjoint class. In other words, there always exists a $\mathcal{C}$-symmetric operator, which is not $\mathcal{C}$-selfadjoint. So, there are the following problems to investigate.

D1: Characterize unbounded weighted composition operators, which are $\mathcal{C}$-selfadjoint.

D2: Characterize unbounded weighted composition operators, which are $\mathcal{C}$-symmetric.

For the necessity of $\mathbf{D} 1$, we apply the symmetric condition $W_{\psi, \varphi} \mathcal{C}=\mathcal{C} W_{\psi, \varphi}^{*}$ to kernel functions, since these functions always belong to $\operatorname{dom}\left(W_{\psi, \varphi}^{*}\right)$. Concerning the sufficiency, it requires more complicated techniques related to domains as well as adjoints of unbounded operators.

In general, kernel functions may not belong to the domain $\operatorname{dom}\left(W_{\psi, \varphi}\right)$. Therefore, problem D2 is surely more difficult than D1.

As a conclusion, we hope that the problems above could be a starting point for new directions in this topic. 


\section{Bibliography}

[1] P. S. Bourdon and S. W. Noor. Complex symmetry of invertible composition operators. J. Math. Anal. Appl., 429(1): 105-110, 2015. [Cited on page 6.]

[2] B. J. Carswell, B. D. MacCluer, and A. Schuster. Composition operators on the Fock space. Acta Sci. Math. (Szeged), 69(3-4): 871-887, 2003. [Cited on page 11.]

[3] C. C. Cowen and B. D. MacCluer. Composition operators on spaces of analytic functions. Studies in Advanced Mathematics. CRC Press, Boca Raton, FL, 1995. [Cited on pages 5, 119, and 120.]

[4] K. J. Engel and R. Nagel. One-parameter semigroups for linear evolution equations. Graduate Texts in Mathematics, 194. Springer-Verlag, New York, 2000. [Cited on pages 8, 49, 50, 63, 72, and 75.]

[5] Y. Gao and Z. Zhou. Complex symmetric composition operators with automorphic symbols. Arxiv preprint, arXiv:1607.04801v3 [math.FA], 31 Jul 2016. [Cited on page 6.]

[6] Y. Gao and Z. Zhou. Complex symmetric composition operators induced by linear fractional transformations. Arxiv preprint, arXiv:1608.01288v3 [math.FA], 28 Jan 2017. [Cited on page 6.] 
[7] S. R. Garcia and C. Hammond. Which weighted composition operators are complex symmetric? Oper. Theory Adv. Appl., 236: 171-179, 2014. [Cited on pages 5, 6, 17, 31, and 37.

[8] S. R. Garcia, E. Prodan, and M. Putinar. Mathematical and physical aspects of complex symmetric operators. J. Phys. A, 47(35): 353001, 54 pp, 2014. [Cited on page 1.]

[9] S. R. Garcia and M. Putinar. Complex symmetric operators and applications. Trans. Amer. Math. Soc., 358(3): 1285-1315, 2006. [Cited on page 1.]

[10] S. R. Garcia and M. Putinar. Complex symmetric operators and applications II. Trans. Amer. Math. Soc., 359(8): 3913-3931, 2007. [Cited on page1.]

[11] I. M. Glazman. An analogue of the extension theory of Hermitian operators and a non-symmetric one-dimensional boundary problem on a half-axis (Russian). Dokl. Akad. Nauk SSSR (N.S.), 115: 214-216, 1957. [Cited on page4.]

[12] I. M. Glazman. Direct methods of qualitative spectral analysis of singular differential operators. Translated from the Russian by the IPST staff. Israel Program for Scientific Translations, Jerusalem, 1965. [Cited on page 4.]

[13] K. H. Izuchi. Cyclic vectors in the Fock space over the complex plane. Proc. Amer. Math. Soc., 133(12): 3627-3630, 2005. [Cited on pages 23, 37, and 73.]

[14] F. Jafari, T. Tonev, E. Toneva, and K. Yale. Holomorphic flows, cocycles, and coboundaries. Michigan Math. J., 44(2): 239-253, 1997. [Cited on page 58.]

[15] S. Jung, Y. Kim, E. Ko, and J. E. Lee. Complex symmetric weighted composition operators on $H^{2}(\mathbb{D})$. J. Funct. Anal., 267(2): 323-351, 2014. [Cited on pages 6, 17, and 37.] 
[16] I. Knowles. On the boundary conditions characterizing J-selfadjoint extensions of J-symmetric operators. J. Differential Equations, 40(2): 193-216, 1981. [Cited on page 4.]

[17] T. Le. Self-adjoint, unitary, and normal weighted composition operators in several variables. J. Math. Anal. Appl., 395(2): 596-607, 2012. [Cited on page 85 .]

[18] T. Le. Normal and isometric weighted composition operators on the Fock space. Bull. Lond. Math. Soc., 46(4): 847-856, 2014. [Cited on pages 10, 11 , 115 , and 118.]

[19] S. K. Narayan, D. Sievewright, and D. Thompson. Complex symmetric composition operators on $H^{2}$. J. Math. Anal. Appl., 443(1): 625-630, 2016. [Cited on page 6.]

[20] J. R. Partington. Linear operators and linear systems. An analytical approach to control theory. London Mathematical Society Student Texts, 60. Cambridge University Press, Cambridge, 2004. [Cited on page 8.]

[21] D. Race. The theory of J-selfadjoint extensions of J-symmetric operators. $J$. Differential Equations, 57(2): 258-274, 1985. [Cited on page 4]

[22] M. Reed and B. Simon. Methods of modern mathematical physics. I. Functional analysis. Academic Press, Inc, New York, 1980. [Cited on pages viii] ix 44.52. 55. and 119.]

[23] V. Scheidemann. Introduction to complex analysis in several variables. Birkhäuser Verlag, Basel, 2005. [Cited on page 37.]

[24] K. Schmüdgen. Unbounded self-adjoint operators on Hilbert space. Graduate Texts in Mathematics, 265. Springer, Dordrecht, 2012. [Cited on pages ix] 3. 48, and 56.] 
[25] A. G. Siskakis. Semigroups of composition operators on spaces of analytic functions, a review. In Studies on composition operators (Laramie, WY, 1996), volume 213 of Contemp. Math., pages 229-252. Amer. Math. Soc., Providence, RI, 1998. [Cited on page 59.]

[26] S. Ueki. Weighted composition operators on some function spaces of entire functions. Bull. Belg. Math. Soc. Simon Stevin, 17(2): 343-353, 2010. [Cited on page 115.]

[27] M. Wang and X. Yao. Complex symmetry of weighted composition operators in several variables. Internat. J. Math., 27(2): 1650017, 14 pp, 2016. [Cited on page 6.]

[28] L. Zhao. Unitary weighted composition operators on the Fock space of $\mathbb{C}^{n}$. Complex Anal. Oper. Theory, 8(2): 581-590, 2014. [Cited on pages 12 and 44.]

[29] L. Zhao. Invertible weighted composition operators on the Fock space of $\mathbb{C}^{n}$. J. Funct. Spaces, 2015. Art. ID 250358. [Cited on page 12.]

[30] L. Zhao. Isometric weighted composition operators on the Fock space of $\mathbb{C}^{n}$. Bull. Korean Math. Soc., 53(6): 1785-1794, 2016. [Cited on pages 11] and 12.]

[31] K. Zhu. Analysis on Fock spaces. Graduate Texts in Mathematics, 263. Springer, New York, 2012. [Cited on page 115.] 
Appendices 



\section{Appendix A}

\section{A study on the Fock spaces $\mathcal{F}^{p}(\mathbb{C})$}

As mentioned in the Introduction, Le gave in [18] complete characterizations for boundedness and compactness of linear weighted composition operators $W_{\psi, \varphi}$ on $\mathcal{F}^{2}(\mathbb{C})$. In [26], Ueki studied for the case $W_{\psi, \varphi}: \mathcal{F}^{p}(\mathbb{C}) \rightarrow \mathcal{F}^{\infty}(\mathbb{C})$, where $0<$ $p \leq \infty$. In this section, we investigate boundedness and compactness for operators $W_{\psi, \varphi}: \mathcal{F}^{p}(\mathbb{C}) \rightarrow \mathcal{F}^{p}(\mathbb{C})$, where $0<p<\infty$. Our results contain the corresponding results for $\mathcal{F}^{2}(\mathbb{C})$ as a particular case.

We recall basic facts of the Fock spaces, whose proofs can be found in the monograph [31]. For $p \in(0, \infty)$, the Fock space $\mathcal{F}^{p}(\mathbb{C})$ is defined as the space of all $f \in \operatorname{Hol}(\mathbb{C})$ for which

$$
\|f\|_{p}=\left(\frac{p}{2 \pi} \int_{\mathbb{C}}|f(z)|^{p} e^{-\frac{p|z|^{2}}{2}} d V(z)\right)^{1 / p}<\infty,
$$

where $d V$ is the Lebesgue measure on $\mathbb{C}$, while the space $\mathcal{F}^{\infty}(\mathbb{C})$ consists of all $f \in \operatorname{Hol}(\mathbb{C})$ for which

$$
\|f\|_{\infty}=\sup _{z \in \mathbb{C}}|f(z)| e^{-\frac{|z|^{2}}{2}}<\infty .
$$

As is well known, for $p \in(0,1), \mathcal{F}^{p}(\mathbb{C})$ just is a complete metric space with the distance $d(f, g)=\|f-g\|_{p}^{p}$, but for $p \in[1, \infty]$ it becomes a Banach space with norm $\|\cdot\|_{p}$. Note that inequality 1.7 also holds on the Fock spaces $\mathcal{F}^{p}(\mathbb{C})$ with $0<p \leq \infty$. Namely,

$$
|h(z)| \leq\|h\|_{p} e^{\frac{|z|^{2}}{2}}, \quad \forall z \in \mathbb{C}, \forall h \in \mathcal{F}^{p}(\mathbb{C}) .
$$


In addition, for $0<p<\infty$,

$$
\lim _{|z| \rightarrow \infty}|h(z)| e^{\frac{-|z|^{2}}{2}}=0, \quad \forall h \in \mathcal{F}^{p}(\mathbb{C}) .
$$

For $1<p<\infty$, the dual space $\left(\mathcal{F}^{p}(\mathbb{C})\right)^{*}$ of $\mathcal{F}^{p}(\mathbb{C})$ can be identified with the space $\mathcal{F}^{q}(\mathbb{C})$, under the duality pairing

$$
\frac{1}{\pi} \int_{\mathbb{C}} f(z) \overline{g(z)} e^{-|z|^{2}} d V(z),
$$

where $1 / p+1 / q=1$, while for $0<p \leq 1$, it is identified with the space $\mathcal{F}^{\infty}(\mathbb{C})$. Thus, $\mathcal{F}^{p}(\mathbb{C})$ is a reflexive Banach space if $1<p<\infty$.

\section{A.1 Boundedness on $\mathcal{F}^{p}(\mathbb{C})$}

In this part, we give another proof for Proposition 1.4.1(1) without using (1.8). Surprisingly, the techniques used here allow us to generalize Proposition 1.4.1(1) to the Fock spaces $\mathcal{F}^{p}(\mathbb{C})$ with $0<p<\infty$.

- For the case when $\varphi$ is non-constant, we obtain the following result.

Proposition A.1.1. Let $0<p<\infty$, let $\varphi \not \equiv$ const, $\psi \not \equiv \mathbf{0}$ be two entire functions. Then the linear weighted composition operator $W_{\psi, \varphi}: \mathcal{F}^{p}(\mathbb{C}) \rightarrow \mathcal{F}^{p}(\mathbb{C})$ is bounded if and only if $M(\psi, \varphi)<\infty$.

In this case, $\varphi(z)=a z+b$ with $0<|a| \leq 1$, and

$$
\begin{gathered}
\sqrt{M_{z}(\psi, \varphi)} \leq\left\|W_{\psi, \varphi} k_{\varphi(z)}\right\|_{p} \leq\left\|W_{\psi, \varphi}\right\|, \forall z \in \mathbb{C}, \\
\sqrt{M(\psi, \varphi)} \leq\left\|W_{\psi, \varphi}\right\| \leq \frac{\sqrt{M(\psi, \varphi)}}{|a|^{2 / p}} .
\end{gathered}
$$

Proof. - Suppose that $W_{\psi, \varphi}$ is bounded, we have

$$
\left\|W_{\psi, \varphi}(g)\right\|_{p} \leq\left\|W_{\psi, \varphi}\right\| \cdot\|g\|_{p}, \forall g \in \mathcal{F}^{p}(\mathbb{C}) .
$$

Then for every $z, u \in \mathbb{C}$, we have

$$
\begin{aligned}
|\psi(z)| \cdot\left|e^{\bar{u} \varphi(z)-\frac{|u|^{2}}{2}}\right| e^{-\frac{|z|^{2}}{2}} & \left.=\left|\left(W_{\psi, \varphi}\left(k_{u}\right)\right)(z)\right| e^{-\frac{|z|^{2}}{2}} \leq\left\|W_{\psi, \varphi}\left(k_{u}\right)\right\|_{p} \quad \text { (by } \mathrm{A} .1\right) \\
& \leq\left\|W_{\psi, \varphi}\right\| \cdot\left\|k_{u}\right\|_{p}=\left\|W_{\psi, \varphi}\right\| \quad \text { (since } k_{u} \text { is a unit vector). }
\end{aligned}
$$


In particular, letting $u=\varphi(z)$, the last inequality becomes $\sqrt{M_{z}(\psi, \varphi)}$, and hence, A.3 holds. Then taking the supremum by $z$ over $\mathbb{C}$ yields the first estimate of A.4.

- Conversely, suppose that $M(\psi, \varphi)<\infty$. By Lemma 1.4.2, $\varphi(z)=a z+b$ with $|a| \leq 1$, and furthermore, $0<|a| \leq 1$ (since $\varphi$ is a non-constant function).

For every $f \in \mathcal{F}^{p}(\mathbb{C})$, we have

$$
\begin{aligned}
\left\|W_{\psi, \varphi}(f)\right\|_{p} & =\left(\frac{p}{2 \pi} \int_{\mathbb{C}} M_{z}(\psi, \varphi)^{p / 2}|f(\varphi(z))|^{p} e^{-\frac{p|\varphi(z)|^{2}}{2}} d V(z)\right)^{1 / p} \\
& \leq \sqrt{M(\psi, \varphi)}\left(\frac{p}{2 \pi} \int_{\mathbb{C}}|f(\varphi(z))|^{p} e^{-\frac{p|\varphi(z)|^{2}}{2}} d V(z)\right)^{1 / p} \\
& \left.=\frac{\sqrt{M(\psi, \varphi)}}{|a|^{2 / p}}\|f\|_{p} \quad \text { (by changing the variables } u=\varphi(z)\right),
\end{aligned}
$$

which gives (A.4).

- For the case when $\varphi$ is constant, our characterization for boundedness is simpler.

Proposition A.1.2. Let $0<p<\infty$, let $\varphi$ be a constant function with $\varphi \equiv b$, and let $\psi \not \equiv \mathbf{0}$ be an entire function. Then the linear weighted composition operator $W_{\psi, \varphi}: \mathcal{F}^{p}(\mathbb{C}) \rightarrow \mathcal{F}^{p}(\mathbb{C})$ is bounded if and only if $\psi \in \mathcal{F}^{p}(\mathbb{C})$.

Moreover, we have

$$
\sqrt{M(\psi, \varphi)} \leq\left\|W_{\psi, \varphi}\right\| \leq\|\psi\|_{p} e^{\frac{|b|^{2}}{2}}
$$

Proof. • Suppose that $W_{\psi, \varphi}$ is bounded. Then $\psi=W_{\psi, \varphi}(\mathbf{1}) \in \mathcal{F}^{p}(\mathbb{C})$.

- Conversely, suppose $\psi \in \mathcal{F}^{p}(\mathbb{C})$. For every $f \in \mathcal{F}^{p}(\mathbb{C})$, by A.1), we have

$$
\left\|W_{\psi, \varphi}(f)\right\|_{p}=|f(b)| \cdot\|\psi\|_{p} \leq\|\psi\|_{p} e^{\frac{|b|^{2}}{2}}\|f\|_{p} .
$$

The proof is complete.

- Propositions A.1.1 A.1.2 can be rewritten in a common statement as follows.

Theorem A.1.3. Let $0<p<\infty$, and let $\psi, \varphi$ be two entire functions with $\psi \not \equiv \mathbf{0}$. The linear weighted composition operator $W_{\psi, \varphi}: \mathcal{F}^{p}(\mathbb{C}) \rightarrow \mathcal{F}^{p}(\mathbb{C})$ is bounded if and only if 
1. $\psi \in \mathcal{F}^{p}(\mathbb{C})$;

2. $M(\psi, \varphi)<\infty$.

Moreover, in this case, $\varphi(z)=a z+b$ with $|a| \leq 1$.

Proof. - Suppose that the operator $W_{\psi, \varphi}: \mathcal{F}^{p}(\mathbb{C}) \rightarrow \mathcal{F}^{p}(\mathbb{C})$ is bounded. We consider two cases of $\varphi$.

- If $\varphi \not \equiv$ const, then by Proposition A.1.1, we have (2), that is $M(\psi, \varphi)<\infty$, and $\varphi(z)=a z+b$ with $0<|a| \leq 1$. By arguments similar to those used in Proposition 2.1.9, we have $\psi \in \mathcal{F}^{p}(\mathbb{C})$, which gives (1).

- If $\varphi \equiv b$ a constant function, then by Proposition A.1.2, we have (1), that is $\psi \in \mathcal{F}^{p}(\mathbb{C})$. By arguments similar to those used in Proposition 2.1.8, we have $M(\psi, \varphi)<\infty$, which gives $(2)$.

- Conversely, suppose that (1)-(2) hold. By Propositions A.1.1 A.1.2, the operator $W_{\psi, \varphi}: \mathcal{F}^{p}(\mathbb{C}) \rightarrow \mathcal{F}^{p}(\mathbb{C})$ is bounded.

\section{A.2 Compactness on $\mathcal{F}^{p}(\mathbb{C})$}

In this part, we generalize Proposition $1.4 .1(2)$ to the Fock spaces $\mathcal{F}^{p}(\mathbb{C})$ with $1<p<\infty$. It should be noted that techniques here are completely different from what Le used in [18].

As introduced in Section 1.4, the Fock space $\mathcal{F}^{p}(\mathbb{C})$ is reflexive in the case when $1<p<\infty$. So, we can make use of the following criterion for compactness.

Proposition A.2.1. Let $1<p<\infty$. A bounded linear operator $\mathcal{Q}$ is compact on $\mathcal{F}^{p}(\mathbb{C})$ if and only if $\lim _{m \rightarrow \infty}\left\|\mathcal{Q} g_{m}-\mathcal{Q} g\right\|_{p} \rightarrow 0$ whenever $g_{m} \rightarrow g$ weakly.

The following result is a criterion for the weak convergence on the Fock spaces $\mathcal{F}^{p}(\mathbb{C})$ with $1<p<\infty$.

Proposition A.2.2. Let $1<p<\infty$. Then a sequence $\left(g_{m}\right)$ converges weakly to 0 in $\mathcal{F}^{p}(\mathbb{C})$ if and only if it is

1. bounded in the norm of $\mathcal{F}^{p}(\mathbb{C})$; 
2. uniformly convergent to 0 on compact subsets of $\mathbb{C}$.

Proof. • Suppose that the sequence $\left(g_{m}\right)$ converges weakly to 0 in $\mathcal{F}^{p}(\mathbb{C})$. By 3 , Corollary 1.3], it is bounded in the norm of $\mathcal{F}^{p}(\mathbb{C})$, and converges pointwise. We must prove that $\left(g_{m}\right)$ converges uniformly on compact subsets of $\mathbb{C}$ to 0 . By the Arzela-Ascoli theorem (see [22, p 30]), it suffices to show that $\left(g_{m}\right)$ is equicontinuous on compact subsets.

For convenience, we denote by

$$
B[u, R]:=\{z \in \mathbb{C}:|z-u| \leq R\}, \quad S(u, R)=\{z:|z-u|=R\}
$$

Putting $L:=\sup _{m \in \mathbb{N}}\left\|g_{m}\right\|_{p}$. Fix $R>0$ and let $z=R e^{i \theta}$. For every $u \in S(z, 1)$, we have by $\mathrm{A} .1$ )

$$
\left|g_{m}(u)\right| \leq e^{|u|^{2} / 2}\left\|g_{m}\right\|_{p} \leq L e^{(|u-z|+|z|)^{2} / 2}=L e^{(1+R)^{2} / 2} .
$$

By the Cauchy integral formula, we have

$$
g_{m}^{\prime}(z)=\frac{1}{2 \pi i} \int_{S(z, 1)} \frac{g_{m}(u) d u}{(u-z)^{2}},
$$

which implies that

$$
\left|g_{m}^{\prime}(z)\right| \leq L e^{(1+R)^{2} / 2}, \quad \forall z \in S(0, R)
$$

Note that since $g_{m}^{\prime}$ is entire, by the Maximum Modulus Principle, the maximum value of $\left|g_{m}^{\prime}\right|$ on $B[0, R]$ occurs on the boundary $S(0, R)$. Hence,

$$
\left|g_{m}^{\prime}(w)\right| \leq L e^{(1+R)^{2} / 2}, \quad \forall w \in B[0, R]
$$

On the other hand, from the fact that

$$
g_{m}(\omega)-g_{m}(u)=\int_{u}^{\omega} g_{m}^{\prime}(\xi) d \xi, \quad \forall \omega, u \in B[0, R]
$$

we estimate $\left|g_{m}(\omega)-g_{m}(u)\right| \leq L e^{(1+R)^{2} / 2}|\omega-u|$. This means that $\left(g_{m}\right)$ is equicontinuous. 
Let $K$ be an arbitrary compact set in $\mathbb{C}$. Then it is bounded. This means that there exists $R=R(K)>0$ such that $K \subseteq B[0, R]$. As proved, the sequence $\left(g_{m}\right)$ is equicontinuous on $B[0, R]$. Thus, it also is equicontinuous on $K$.

- Conversely, suppose that the sequence $\left(g_{m}\right)$ is bounded in the norm of $\mathcal{F}^{p}(\mathbb{C})$, and converges uniformly on compact subsets of $\mathbb{C}$ to 0 . Clearly, uniform convergence implies pointwise convergence. Again by [3, Corollary 1.3], the sequence $\left(g_{m}\right)$ converges weakly to 0 in $\mathcal{F}^{p}(\mathbb{C})$.

An immediate consequence of Proposition A.2.2 is the following.

Corollary A.2.3. Let $1<p<\infty, a \in \mathbb{C} \backslash\{0\}, b \in \mathbb{C}$ and $\left(w_{m}\right)$ be a sequence in $\mathbb{C}$ with $\lim _{m \rightarrow \infty}\left|w_{m}\right|=\infty$. Then the sequence $\left(g_{m}\right)$, where $g_{m}=k_{a w_{m}+b}, \forall m \geq 1$, converges weakly in $\mathcal{F}^{p}(\mathbb{C})$ to 0 .

Proof. To show that $\left(g_{m}\right)$ converges weakly to 0, we make use of Proposition A.2.2. The sequence $\left(g_{m}\right)$ is bounded in the norm of $\mathcal{F}^{p}(\mathbb{C})$, since

$$
\left\|g_{m}\right\|_{p}=\left\|k_{a w_{m}+b}\right\|_{p}=1, \quad \forall m \in \mathbb{N}
$$

Let $K$ be a compact set in $\mathbb{C}$. Then there exists a $R=R(K)>0$ such that $K \subseteq B[0, R]$. For every $z \in K$, we have

$$
\left|g_{m}(z)\right|=e^{-\left|a w_{m}+b\right|^{2} / 2} e^{\operatorname{Re} z\left(\overline{a w_{m}+b}\right)} \leq e^{-\left|a w_{m}+b\right|^{2} / 2} e^{\left|a w_{m}+b\right| R}
$$

which, as $a \neq 0$ and $\lim _{m \rightarrow \infty}\left|a w_{m}+b\right|=\infty$, converges uniformly on $K$.

Now we formulate the criterion for compactness of $W_{\psi, \varphi}$. Just as boundedness, we consider two cases as follows.

- For the case when $\varphi$ is a non-constant function, we have the following result.

Proposition A.2.4. Let $1<p<\infty$, and $W_{\psi, \varphi}$ a linear weighted composition operator on $\mathcal{F}^{p}(\mathbb{C})$ induced by two entire functions $\varphi \not \equiv$ const, $\psi \neq \equiv \mathbf{0}$. Then the operator $W_{\psi, \varphi}: \mathcal{F}^{p}(\mathbb{C}) \rightarrow \mathcal{F}^{p}(\mathbb{C})$ is compact if and only if $\lim _{|z| \rightarrow \infty} M_{z}(\psi, \varphi)=0$.

Moreover, in this case, $\varphi(z)=a z+b$ with $0<|a|<1$. 
Proof. • Suppose that $W_{\psi, \varphi}$ is compact. Then it is bounded, and hence by Theorem A.1.3,

$$
\psi \in \mathcal{F}^{p}(\mathbb{C}), \quad M(\psi, \varphi)<\infty, \quad \text { and } \quad \varphi(z)=a z+b,|a| \leq 1
$$

Furthermore, $a \neq 0$ since $\varphi$ is a non-constant function.

We prove the conclusion by a contradiction. Assume in a contrary that there exist $\varepsilon_{0}>0$ and a sequence $\left(w_{m}\right)$ such that

$$
\lim _{m \rightarrow \infty}\left|w_{m}\right|=\infty, \quad \limsup _{m \rightarrow \infty} M_{w_{m}}(\psi, \varphi) \geq \varepsilon_{0}
$$

On the other hand, by A.3 and by Corollary A.2.3.

$$
\sqrt{M_{w_{m}}(\psi, \varphi)} \leq\left\|W_{\psi, \varphi} k_{\varphi\left(w_{m}\right)}\right\|_{p}, \forall m \in \mathbb{N} \Longrightarrow \lim _{m \rightarrow \infty} M_{w_{m}}(\psi, \varphi)=0
$$

which contradicts A.5.

- Conversely, suppose that $\lim _{|z| \rightarrow \infty} M_{z}(\psi, \varphi)=0$. By Lemma 1.4.2, $\varphi(z)=a z+b$ with $|a|<1$, and furthermore, $a \neq 0$ since $\varphi$ is a non-constant function. Clearly, $M(\psi, \varphi)$ is finite, and so by Proposition A.1.1. $W_{\psi, \varphi}$ is bounded on $\mathcal{F}^{p}(\mathbb{C})$.

Let $\left(g_{m}\right) \subset \mathcal{F}^{p}(\mathbb{C})$ be a sequence converging weakly to 0 in $\mathcal{F}^{p}(\mathbb{C})$. By Proposition A.2.2, it is bounded and converges to 0 uniformly on compact subsets of $\mathbb{C}$. Put $L:=\sup _{m \in \mathbb{N}}\left\|g_{m}\right\|_{p}<\infty$. By Proposition A.2.1, it suffices to show that $W_{\psi, \varphi}\left(g_{m}\right)$ converges to 0 in $\mathcal{F}^{p}(\mathbb{C})$-norm as $m \rightarrow \infty$. Set

$$
F(u):=M_{\frac{u-b}{a}}(\psi, \varphi), u \in \mathbb{C} .
$$

Note that since $a \neq 0$,

$$
\lim _{|z| \rightarrow \infty} M_{z}(\psi, \varphi)=0 \Longleftrightarrow \lim _{|u| \rightarrow \infty} F(u)=0
$$

In addition,

$$
F(\varphi(z))=M_{z}(\psi, \varphi), \quad F(u) \leq M(\psi, \varphi)
$$

By changing the variables $u=\varphi(z)$, we get

$$
\left\|W_{\psi, \varphi}\left(g_{m}\right)\right\|_{p}^{p}=\frac{p}{2 \pi|a|^{2}} \int_{\mathbb{C}} F(u)^{p / 2}\left|g_{m}(u)\right|^{p} e^{-\frac{p|u|^{2}}{2}} d V(u) .
$$


Given $\varepsilon>0$. Since $\lim _{|u| \rightarrow \infty} F(u)=0$, there exists $R=R(\varepsilon)$ such that

$$
\frac{L^{p}}{|a|^{2}} \sup _{|u|>R} F(u)^{p / 2}<\frac{\varepsilon^{p}}{2} .
$$

With such $R$, we estimate

$$
\begin{aligned}
\int_{|u|>R} F(u)^{p / 2}\left|g_{m}(u)\right|^{p} e^{-\frac{p|u|^{2}}{2}} d V(u) & \leq\left(\int_{u \mid>R}\left|g_{m}(u)\right|^{p} e^{-\frac{p|u|^{2}}{2}} d V(u)\right) \sup _{|u|>R} F(u)^{p / 2} \\
& \leq\left(\int_{\mathbb{C}}\left|g_{m}(u)\right|^{p} e^{-\frac{p|u|^{2}}{2}} d V(u)\right) \sup _{|u|>R} F(u)^{p / 2} \\
& =\frac{2 \pi\left\|g_{m}\right\|_{p}^{p}}{p} \sup _{|u|>R} F(u)^{p / 2} \\
& \leq \frac{\pi \varepsilon^{p}|a|^{2}}{p}(\text { by }(\mathrm{A.6})) .
\end{aligned}
$$

Therefore,

$$
\begin{aligned}
\left\|W_{\psi, \varphi}\left(g_{m}\right)\right\|_{p}^{p} & =\frac{p}{2 \pi|a|^{2}}\left(\int_{|u| \leq R}+\int_{|u|>R}\right) F(u)^{p / 2}\left|g_{m}(u)\right|^{p} e^{-\frac{p|u|^{2}}{2}} d V(u) \\
& \leq \frac{p}{2 \pi|a|^{2}} \int_{|u| \leq R} F(u)^{p / 2}\left|g_{m}(u)\right|^{p} e^{-\frac{p|u|^{2}}{2}} d V(u)+\frac{\varepsilon^{p}}{2}
\end{aligned}
$$

Since $F(u) \leq M(\psi, \varphi)$, we have

$$
\begin{aligned}
\left\|W_{\psi, \varphi}\left(g_{m}\right)\right\|_{p}^{p} & \leq \frac{p M(\psi, \varphi)^{p / 2}}{2 \pi|a|^{2}} \int_{|u| \leq R}\left|g_{m}(u)\right|^{p} e^{-\frac{p|u|^{2}}{2}} d V(u)+\frac{\varepsilon^{p}}{2} \\
& \leq \frac{p M(\psi, \varphi)^{p / 2}}{2 \pi|a|^{2}} \int_{|u| \leq R} e^{-\frac{p|u|^{2}}{2}} d V(u) \cdot \max _{|u| \leq R}\left|g_{m}(u)\right|^{p}+\frac{\varepsilon^{p}}{2} .
\end{aligned}
$$

Since $\left(g_{m}\right)$ converges to 0 uniformly on compact subsets of $\mathbb{C}$, the integral on the right-hand side can be arbitrarily small, by choosing $m$ sufficiently large. Consequently, $W_{\psi, \varphi}\left(g_{m}\right)$ converges to 0 in the norm of $\mathcal{F}^{p}(\mathbb{C})$ as $m \rightarrow \infty$.

- For the second case when $\varphi$ is a constant function, it turns out that a bounded linear weighted composition operator $W_{\psi, \varphi}$ must necessarily be compact. 
Proposition A.2.5. Let $1<p<\infty$, and $W_{\psi}$ a linear weighted composition induced by an entire function $\psi \neq \equiv \mathbf{0}$ and a constant function $\varphi \equiv b$.

1. The operator $W_{\psi, \varphi}: \mathcal{F}^{p}(\mathbb{C}) \rightarrow \mathcal{F}^{p}(\mathbb{C})$ is compact;

2. The operator $W_{\psi, \varphi}: \mathcal{F}^{p}(\mathbb{C}) \rightarrow \mathcal{F}^{p}(\mathbb{C})$ is bounded;

3. The function $\psi$ satisfies $\psi \in \mathcal{F}^{p}(\mathbb{C})$.

Proof. Clearly, $(1) \Longrightarrow(2)$ by definition, while $(2) \Longleftrightarrow(3)$ by Proposition A.1.2.

To prove that $(3) \Longrightarrow(1)$, we suppose that $(3)$ holds. Let $\left(g_{m}\right) \subset \mathcal{F}^{p}(\mathbb{C})$ be a sequence converging weakly to 0 in $\mathcal{F}^{p}(\mathbb{C})$. By Proposition A.2.2, it converges to 0 pointwise. Since $\psi \in \mathcal{F}^{p}(\mathbb{C})$, we have

$$
\left\|W_{\psi, \varphi} g_{m}\right\|_{p}=\left|g_{m}(b)\right| \cdot\|\psi\|_{p}
$$

which tends to 0 as $m \rightarrow \infty$. The proof is complete.

- Combining Propositions A.2.4 A.2.5, we have the following theorem.

Theorem A.2.6. Let $1<p<\infty$, and $W_{\psi, \varphi}$ a linear weighted composition operator on $\mathcal{F}^{p}(\mathbb{C})$ induced by two entire functions $\psi, \varphi$ with $\psi \not \equiv \mathbf{0}$. Then the operator $W_{\psi, \varphi}: \mathcal{F}^{p}(\mathbb{C}) \rightarrow \mathcal{F}^{p}(\mathbb{C})$ is compact if and only if

1. $\psi \in \mathcal{F}^{p}(\mathbb{C})$;

2. $\lim _{|z| \rightarrow \infty} M_{z}(\psi, \varphi)=0$.

Moreover, in this case, $\varphi(z)=a z+b$ with $|a|<1$.

Proof. • Suppose that the operator $W_{\psi, \varphi}: \mathcal{F}^{p}(\mathbb{C}) \rightarrow \mathcal{F}^{p}(\mathbb{C})$ is compact. As for boundedness, we divide into two cases.

- If $\varphi \not \equiv$ const, then by Proposition A.2.4, we have (2), which implies that $M(\psi, \varphi)$ is finite. By arguments similar to those used in Proposition 2.1.9, we can show that $\psi \in \mathcal{F}^{p}(\mathbb{C})$, which gives (1).

- If $\varphi \equiv b$ a constant function, then by Proposition A.2.5, we have (1), that is $\psi \in \mathcal{F}^{p}(\mathbb{C})$. By $\mathrm{A} .2, M_{z}(\psi, \varphi)$ converges to 0 as $|z| \rightarrow \infty$.

- Conversely, suppose that (1)-(2) hold. By Propositions A.2.4 A.2.5, the operator $W_{\psi, \varphi}$ is compact. 